\title{
Nonlinear Equations of Motion of L-Shaped Beam Structures
}

\author{
Fotios Georgiades \\ School of Engineering, College of Science, University of Lincoln, Lincoln, UK \\ fgeorgiadis@lincoln.ac.uk \\ Corresponding author: Address University of Lincoln, College of Science, School of Engineering, \\ Brayford Pool, Lincoln, UK, LN6 7TS. Phone: +44-15-22-83-79-52.
}

\begin{abstract}
:
An L-shaped inextensional isotropic Euler-Bernoulli beam structure is considered in this paper and all the nonlinear equations of motion are derived up to and including second order nonlinearity. The associated nonlinear boundary conditions have also been derived up to and including second order terms. The global displacements of the secondary beam have been used in the equations of motion and the rotary inertia terms have also been considered. It has been demonstrated that the nonlinear equations couple the in-plane and the out-of-plane motions. It is also shown that the associated linear mode shapes are orthogonal to each other, which is essential for discretization of the equations of motion. Considering that the linear global displacements have been used in deriving the equations of motion then these equations can easily be discretised by projecting the dynamics onto the infinite linear mode shape basis, for reduce order modelling. Completion of this work is judged to have been essential in order to be able to perform nonlinear modal analysis on the L-shaped beam structure. It should be mentioned that this is the only nonlinear model describing the out-of-plane motions of LShaped beam structure which have been neglected so far in the literature. The presented process of deriving the mathematical model of the L-shaped beam structure paves the way for modelling of the nonlinear dynamics of more complicated structures comprised with several elementary substructures considering geometric nonlinearities and this analytical modelling is essential for further reduced order modelling and analysis of the nonlinear dynamics of these models.
\end{abstract}

Keywords: L-shaped beam; nonlinear equations of motion; autoparametric system; nonlinear dynamics.

\section{Highlights:}

- A derivation is given for the nonlinear equations of motion for an L-shaped beam.

- A derivation is also provided for the associated nonlinear boundary conditions and Lagrange multipliers.

-The in-plane equations of motion are shown to be coupled with those describing out-of-plane motion. -It has been proven that both the underlying linear systems of equations are self-adjoint.

-The out-of-plane motions in case of geometric nonlinearities are significant even in examining inplane motions. 


\section{Introduction}

Since the 1960s, the dynamics of L-shaped coupled beams exhibiting nonlinearities and autoparametric coupling have been of interest because of their sensitivity and importance in real engineering structures. As an example it can be mentioned aerostructures such as fins and spars or helicopter subsystems composed of a main body and a tail boom. Coupled beams having an L shape naturally appear in robots when considering their coupled arms, or in satellite solar panels and flexible antennas fixed to an extension arm, and also lately the L-shape beam structure is used as a very simplified model of a tree structure. Another important application is the floating crane which has to operate under various excitation conditions. Despite their relatively structural simplicity these Lshaped structures potentially exhibit many complex dynamical phenomena in the form of intermodal energy pumping, and nonlinear internal, external and autoparametric resonances. Moreover, apart from regular periodic motions it is also possible to observe chaotic responses. The literature shows that early evidence of principal parametric resonance in a simply supported beam subjected to periodic end movements was reported in detail by Bolotin in (1964). Haddow et al (1984) investigated an L-shaped beam structure considering only the in-plane motion of a truncated 2-dof nonlinear model. The theoretical dynamics were confirmed experimentally. Roberts and Cartmell (1984) considered forced vibration of an L-shaped beam structure, and compared theoretical and experimental results of nonlinear modal interactions between a pair of beam modes, with close agreement shown. Bux and Roberts (1986) examined nonlinear vibratory interactions of a forced L-shaped beam structure, and showed the existence of nonsynchronous torsion and bending vibrations, and their analytical solution for three-mode interaction was in good agreement with experimental results. Cartmell and Roberts (1987), also examined simultaneous combination resonances in a parametrically excited nonlinear cantilever beam using first and second order approximations and their comparison of theoretical predictions of stability boundaries was seen to be in a good agreement with their experimental results. Balachandran and Nayfeh (1990a), modelled a nonlinear L-shaped beam with additional tip masses at some points, but they considered only the in-plane bending motions. They also performed a nonlinear modal analysis and studied selected nonlinear resonances. Balachandran and Nayfeh in (1990b), expanded the dynamic analysis of in-plane motions of L-shaped beam structures to composite materials. These authors observed experimental nonplanar motions because of the presence of the torsional mode in the response, and this effect was found to be qualitatively different to that encountered in metallic structures. Warminski et al. (2008), using local displacements, formulated the third order partial differential nonlinear equations for an L-shaped beam without taking into account the rotary inertia effects, and they also performed numerical and experimental studies. Ozonato et al. (2012), analysed post-buckled chaotic vibrations of an L-shaped beam structure considering only inplane nonlinear bending motions. Nayfeh and Pai (2004), modelled and analysed the dynamics for many cases of autoparametric excitations of nonlinear beams. Georgiades et al. (2013a), used the Lagrange formulation to derive the linear equations of motion for an L-shaped beam structure considering the inextensionality conditions, the rotary inertia terms and also out-of-plane motions. In this study the necessity for rotary inertia terms in out-of-plane bending was confirmed and also it was shown that when considering the linear equations of motion the in-plane motions were well separated from the out-of-plane motions. Linear modal analysis of an L-shaped beam structure was performed in the work of Georgiades et al. (2013b), and the eigenvalue and eigenvector problem was solved analytically for both in-plane and out-of-plane motions, using the global displacements of the secondary beam in the linear regime. Comparison of the analytical results showed a good agreement with those obtained by finite element analysis. In the literature of L-shaped beam structures there is a significant gap whereas the out-of-plane motions of the structure are not studied and this article paves the way of examining these out-of-plane motions in large deformation regime.

The main goal of this paper is to derive the equations of motion of an L-shaped beam structure in order to examine the full three dimensional motions and especially the out-of-plane motions which has been neglected so far in the literature. Also, in prior of examining nonlinear dynamics of this model, on this article, it is shown that the underlying linear modes are orthogonal which is essential for reduced order modelling. In the literature there are nonlinear models restricted to in-plane motion but there are not any nonlinear models describing the out-of-plane motions where these are highly 
significant in cases where there is excitation in out-of-plane motion. Also it is significant in structures when the stiffness coefficients in both directions (in-plane with out-of-plane) have comparable values, and therefore the flexibilities describing the underlying linear motion in both directions also have similar values. To the author's knowledge there is no such mathematical model available in the literature, that describes out-of-plane motion of L-shaped beam structures in large deformation regime. Considering perturbation theory and also the frequency amplitude dependence or frequency energy dependence of the nonlinear systems, the first order (linear) system describes small motions, therefore is valid only for a small range of energies. The second and the third order nonlinear mathematical models enable describing motions with larger amplitudes which correspond to medium and high energy levels respectively. On this article the second order equations of motions are derived, which although the third order nonlinearity is not included, this derivation is quite involved and can be as a benchmark modelling for the derivation of the third order nonlinear system. More precisely, in this article it is derived the nonlinear equations of motion, up to second order, of an L-shaped inextensional beam structure, using global displacements and also considering the rotary inertia terms. It is also obtained the nonlinear boundary conditions which are necessary in order to determine the Lagrange multipliers involved in the main equations of motion. Also it is shown that the underlying linear system is self-adjoint and therefore the linear mode shapes are orthogonal to each other.

Completion of this work, is considered to be essential prior to any further rigorous examination of the nonlinear dynamics of the L-shaped beam in three dimensional space. It is important to mention that other modelling methods such as the use of commercial finite element software are not appropriate for studying the nonlinear vibrations of flexible structures as covered here because they have significant number of degrees of freedom to examine the nonlinear dynamics e.g. by examining the periodic orbits of the system and their bifurcations in a Frequency Energy plot diagram which is rather complicated and sometimes even impossible to identify all the bifurcation points for such a large system. The derived model on this article can be used to derive a reduced order model using the linear normal modes and make the nonlinear dynamics examination rather simpler. Therefore, despite its complex form, an analytical model is a deeply descriptive approach available for the determination and understanding of such a system's regular (normal modes) and chaotic dynamics, and for finding the essential bifurcation points.

\section{Material, geometric configuration and energies}

It is considered Euler-Bernoulli beams made of a linear elastic isotropic material and with constant cross-section with respect to the longitudinal directions $\left(\mathrm{X}_{1}, \mathrm{X}_{2}\right)$ and which are sufficiently thin and long (at least 10 times longer than the thickness, and it is also noted that the shear effects can be neglected). It is also assumed that the beams are inextensional. This assumption is justified in the considered cases, because of the strong boundary conditions fixed at one end and free at the other and with no axial external forcing [Nayfeh and Pai 2004]. In this way the equations that describe the motion can be simplified and the problem is reduced. This assumption has been confirmed by the author through the use of FE analysis by computing ten linear modes of vibration. It was found that the inextensionality condition was significant only for the axial modes of vibration [Georgiades et al. $2013 b]$. In advance of any consideration of a variational formulation for the system as shown in Figure 1, it is defined the curvatures and corresponding angular velocities using local displacements, and so for the primary beam these are as given by Nayfeh and Pai (2004) using three Euler angles, and also by Arafat (1998). It is considered three Euler angles in order to include torsional motion, which, even in the linear case, is coupled with the bending equations for out-of-plane motions [Georgiades et al 2013a]. It should be noted that the coordinate's axes of the beams are the principal inertial axes. Noting that the subscripts refer to the axes shown in Figure 1, and also only the axial terms at the third order of nonlinearity are considered (using the $3 *$ notation for these terms) which lead to second order terms in the main equations of motion for cases of differentiation with respect to these terms,

$$
\rho_{\xi_{1}}=\left\{\rho_{\xi_{1}}^{(1)}\right\}+\left\{\rho_{\xi_{1}}^{(2)}\right\}+\left\{\rho_{\xi_{1}}^{(3 *)}\right\}=\left\{\varphi_{1}^{\prime}\right\}+\left\{v_{1}^{\prime \prime} w_{1}^{\prime}\right\}+\{0\}
$$




$$
\begin{gathered}
\rho_{\eta_{1}}=\left\{\rho_{\eta_{1}}^{(1)}\right\}+\left\{\rho_{\eta_{1}}^{(2)}\right\}+\left\{\rho_{\eta_{1}}^{(3 *)}\right\}=\left\{-w_{1}^{\prime \prime}\right\}+\left\{\varphi_{1} v_{1}^{\prime \prime}\right\}+\left\{u_{1}^{\prime \prime} w_{1}^{\prime}+u_{1}^{\prime} w_{1}^{\prime \prime}\right\}, \\
\rho_{\zeta_{1}}=\left\{\rho_{\zeta_{1}}^{(1)}\right\}+\left\{\rho_{\zeta_{1}}^{(2)}\right\}+\left\{\rho_{\zeta_{1}}^{(3 *)}\right\}=\left\{v_{1}^{\prime \prime}\right\}+\left\{\varphi_{1} w_{1}^{\prime \prime}\right\}+\left\{-u_{1}^{\prime \prime} v_{1}^{\prime}-u_{1}^{\prime} v_{1}^{\prime \prime}\right\}, \\
\omega_{\xi_{1}}=\left\{\omega_{\xi_{1}}^{(1)}\right\}+\left\{\omega_{\xi_{1}}^{(2)}\right\}+\left\{\omega_{\xi_{1}}^{(3 *)}\right\}=\left\{\dot{\varphi}_{1}\right\}+\left\{\dot{v}_{1}^{\prime} w_{1}^{\prime}\right\}+\{0\}, \\
\omega_{\eta_{1}}=\left\{\omega_{\eta_{1}}^{(1)}\right\}+\left\{\omega_{\eta_{1}}^{(2)}\right\}+\left\{\omega_{\eta_{1}}^{(3 *)}\right\}=\left\{\delta_{1}\left(-\dot{w}_{1}^{\prime}\right)\right\}+\left\{\delta_{1}\left(\varphi_{1} \dot{v}_{1}^{\prime}\right)\right\}+\left\{\delta_{1}\left(\dot{u}_{1}^{\prime} w_{1}^{\prime}+u_{1}^{\prime} \dot{w}_{1}^{\prime}\right)\right\}, \\
\omega_{\zeta_{1}}=\left\{\omega_{\zeta_{1}}^{(1)}\right\}+\left\{\omega_{\zeta_{1}}^{(2)}\right\}+\left\{\omega_{\zeta_{1}}^{(3 *)}\right\}=\left\{\delta_{3}\left(\dot{v}_{1}^{\prime}\right)\right\}+\left\{\delta_{3}\left(\varphi_{1} \dot{w}_{1}^{\prime}\right)\right\}+\left\{\delta_{3}\left(-\dot{u}_{1}^{\prime} v_{1}^{\prime}-u_{1}^{\prime} \dot{v}_{1}^{\prime}\right)\right\},
\end{gathered}
$$

whereas, the subscript 1 or 2 refers to the primary and secondary beam respectively, the dot denotes differentiation with respect to time and the dash denotes differentiation with respect to space (in case of subscript 1 then it is with respect to $s_{1}$ otherwise with respect to $s_{2}$ ), and for the secondary beam they are given by,

$$
\begin{gathered}
\rho_{\xi_{2}}=\left\{\rho_{\xi_{2}}^{(1)}\right\}+\left\{\rho_{\xi_{2}}^{(2)}\right\}+\left\{\rho_{\xi_{2}}^{(3 *)}\right\}=\left\{\varphi_{2}^{\prime}\right\}+\left\{v_{2}^{\prime \prime} w_{2}^{\prime}\right\}+\{0\} \\
\rho_{\eta_{2}}=\left\{\rho_{\eta_{2}}^{(1)}\right\}+\left\{\rho_{\eta_{2}}^{(2)}\right\}+\left\{\rho_{\eta_{2}}^{(3 *)}\right\}=\left\{-w_{2}^{\prime \prime}\right\}+\left\{\varphi_{2} v_{2}^{\prime \prime}\right\}+\left\{u_{2}^{\prime \prime} w_{2}^{\prime}+u_{2}^{\prime} w_{2}^{\prime \prime}\right\}, \\
\rho_{\zeta_{2}}=\left\{\rho_{\zeta_{2}}^{(1)}\right\}+\left\{\rho_{\zeta_{2}}^{(2)}\right\}+\left\{\rho_{\zeta_{2}}^{(3 *)}\right\}=\left\{v_{2}^{\prime \prime}\right\}+\left\{\varphi_{2} w_{2}^{\prime \prime}\right\}+\left\{-u_{2}^{\prime \prime} v_{2}^{\prime}-u_{2}^{\prime} v_{2}^{\prime \prime}\right\}, \\
\omega_{\xi_{2}}=\left\{\omega_{\xi_{2}}^{(1)}\right\}+\left\{\omega_{\xi_{2}}^{(2)}\right\}+\left\{\omega_{\xi_{2}}^{(3 *)}\right\}=\left\{\dot{\varphi}_{2}+\delta_{1} \omega_{\eta_{1, C}}^{(1)}\right\}+\left\{\dot{v}_{2}^{\prime} w_{2}^{\prime}+\delta_{1} \omega_{\eta_{1, C}}^{(2)}\right\}+\left\{0+\delta_{1} \omega_{\eta_{1, C}}^{(3)}\right\}= \\
=\left\{\dot{\varphi}_{2}-\delta_{1} \dot{w}_{1 c}^{\prime}\right\}+\left\{\dot{v}_{2}^{\prime} w_{2}^{\prime}+\delta_{1} \varphi_{1 c} \dot{v}_{1 c}^{\prime}\right\}+\left\{0+\delta_{1} \dot{u}_{1 c}^{\prime} w_{1 c}^{\prime}+\delta_{1} u_{1 c}^{\prime} \dot{w}_{1 c}^{\prime}\right\}, \\
\omega_{\eta_{2}}=\left\{\omega_{\eta_{2}}^{(1)}\right\}+\left\{\omega_{\eta_{2}}^{(2)}\right\}+\left\{\omega_{\eta_{2}}^{(3 *)}\right\}= \\
=\left\{\omega_{\zeta_{2}}=\left\{\omega_{\zeta_{2}}^{(1)}\right\}+\left\{\omega_{\zeta_{2}}^{(2)}\right\}+\left\{\omega_{\zeta_{2}}^{(3 *)}\right\}=\right. \\
\left.\left.\delta_{2}+\dot{v}_{2}^{\prime}+\omega_{\zeta_{1, C}}^{(1)}\right)\right\}+\left\{\delta_{3}\left(\varphi_{2} \dot{v}_{2}^{\prime}+\omega_{\zeta_{1, C}}^{(2)}\right)\right\}+\left\{\delta_{3}\left(\dot{u}_{2}^{\prime} w_{2}^{\prime}+u_{2}^{\prime} \dot{w}_{2}^{\prime}+\omega_{\zeta_{1, C}}^{(3 *)}\right)\right\}= \\
\left.\left.\left.=\left\{\dot{v}_{2}^{\prime}+\varphi_{1 c} \dot{w}_{1 c}^{\prime}\right)\right\}+\left\{\dot{v}_{2}^{\prime}+\omega_{\xi_{1, C}}^{(1)}\right)\right\}+\left\{\dot{u}_{2}^{\prime} w_{2}^{\prime}+u_{2}^{\prime} \dot{w}_{2}^{\prime}-\dot{u}_{1 c}^{\prime} v_{1 c}^{\prime}-u_{1 c}^{\prime} \dot{v}_{1 c}^{\prime}\right)\right\} \\
\left.=\left\{\omega_{\xi_{1, C}}^{(2)}\right)\right\}+\left\{\delta_{2}\left(-\dot{u}_{2}^{\prime} v_{2}^{\prime}-u_{2}^{\prime} \dot{v}_{2}^{\prime}\right)\right\}=
\end{gathered}
$$

where $\mathrm{C}$ is the point of connection between the primary and secondary beams. In order to check the effect of rotary inertia terms at the end of the formulation, a switching function is included, in the form of a delta ( $\delta_{i}$, with $\left.i=1-3\right)$, such that omitting the particular rotary inertia term- $j$ requires that $\delta_{j}=0$ otherwise $\delta_{j}=1$. It should be mentioned that even for the out-of-plane linear equations it has been shown necessary to include the rotary inertia terms which couple the torsional motion of secondary beam with the other out-of-plane motions [Georgiades et al. 2013a].

As indicated in Figure 1(a) the deformations of the primary beam $v_{1}, w_{1}, \varphi_{1}, u_{1}$, are bending in $Y_{1}$, bending in $Z_{1}$, and then torsional and axial, respectively. Similarly, as indicated in Figure 1(b) the deformations of the secondary beam $v_{2}, w_{2}, \varphi_{2}, u_{2}$, are bending in $Y_{2}$, bending in $Z_{2}$, and again torsional and axial, respectively.

Translational velocities for the primary beam are given by,

$$
V_{x_{1}}=\dot{u}_{1}, V_{y_{1}}=\dot{v}_{1}, V_{z_{1}}=\dot{w}_{1}
$$


The velocities are expressed in terms of the coordinate set $i_{0 x}, i_{0 y}, i_{0 z}$ of the origin $X_{1} Y_{1} Z_{1}$ of the primary beam. In the case of large deformations (and therefore the nonlinear regime), and in order to define the velocities of the primary beam at point $C$ and express them in the coordinate system $i_{x}, i_{y}$, $\mathrm{i}_{\mathrm{z}}$ of the origin at $\mathrm{C}\left(\mathrm{X}_{2} \mathrm{Y}_{2} \mathrm{Z}_{2}\right)$ of the secondary beam, it is taken into account the deformation of the primary beam.

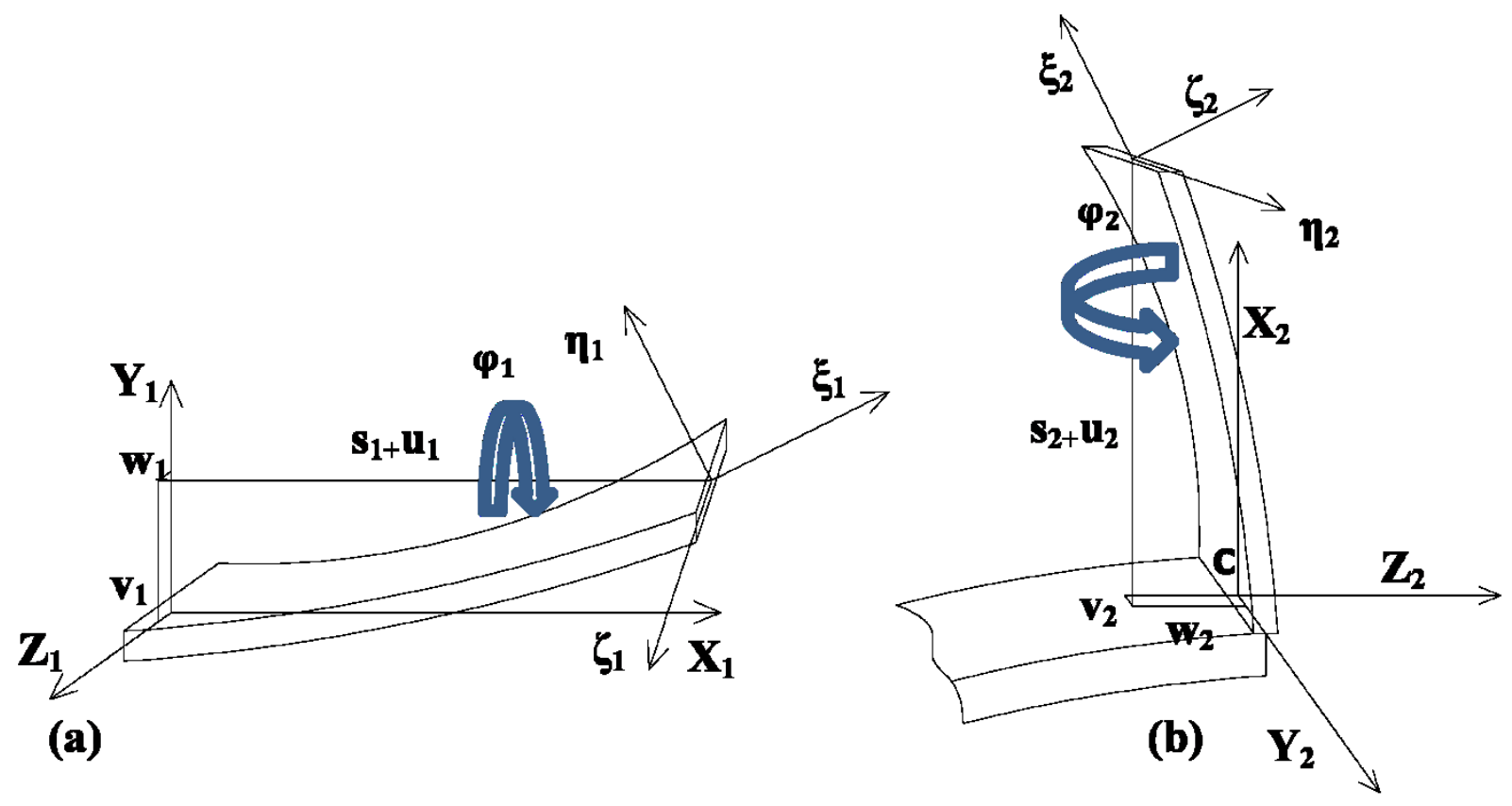

Figure 1. The L-shaped beam structure with an indication of axis orientations and displacements for (a) the primary beam, and (b) the secondary beam.

The relationship between the coordinate systems is extracted from Nayfeh and Pai (2004) for the three Euler angles and is given by,

$$
\left\{\begin{array}{l}
i_{y} \\
i_{z} \\
i_{x}
\end{array}\right\}=\left[A_{i j}\right]\left\{\begin{array}{l}
i_{0 x} \\
i_{0 y} \\
i_{0 z}
\end{array}\right\} \Leftrightarrow\left\{\begin{array}{l}
i_{0 x} \\
i_{0 y} \\
i_{0 z}
\end{array}\right\}=\left[A_{i j}\right]^{T}\left\{\begin{array}{l}
i_{y} \\
i_{z} \\
i_{x}
\end{array}\right\}
$$

for which matrix $A_{i j}$ is defined by,

$$
\left[A_{i j}\right]=\left[\begin{array}{ccc}
1-\frac{w_{1}^{\prime 2}}{2}-\frac{v_{1}^{\prime 2}}{2} & v_{1}^{\prime} & w_{1}^{\prime} \\
-v_{1}^{\prime}-w_{1}^{\prime} \varphi_{1} & 1-\frac{\varphi_{1}^{2}}{2}-\frac{v_{1}^{\prime 2}}{2} & \varphi_{1} \\
-w_{1}^{\prime}+v_{1}^{\prime} \varphi_{1} & -\varphi_{1}-w_{1}^{\prime} v_{1}^{\prime} & 1-\frac{\varphi_{1}^{2}}{2}-\frac{w_{1}^{\prime 2}}{2}
\end{array}\right]
$$

considering nonlinear terms up to the second order of approximation. Therefore using equations (3a-c) with equations (4) and (5) it can be defined the translational velocity of the primary beam at point $\mathrm{C}$ (origin $\mathrm{X}_{2} \mathrm{Y}_{2} \mathrm{Z}_{2}$ ) as, 


$$
\vec{V}_{C}=\left\{\begin{array}{l}
\dot{v}_{1 c}-v_{1 c}^{\prime} \dot{u}_{1 c}+\varphi_{1 c} \dot{w}_{1 c} \\
\dot{w}_{1 c}-\varphi_{1 c} \dot{v}_{1 c}-w_{1 c}^{\prime} \dot{u}_{1 c} \\
\dot{u}_{1 c}+v_{1 c}^{\prime} \dot{v}_{1 c}+w_{1 c}^{\prime} \dot{w}_{1 c}
\end{array}\right\} .
$$

In the secondary beam the translational velocity $V_{2}$ of the centre of the cross section, due to the motion of point $\mathrm{C}$, (the clamped end, in local displacements) can be determined by considering the relative velocity $V_{r}$, and also the translational and angular motions of the origin of $\mathrm{X}_{2} \mathrm{Y}_{2} \mathrm{Z}_{2}$ at point $\mathrm{C}$. Therefore velocity $V_{2}$ in vector form is given by,

$$
\vec{V}_{2}=\vec{V}_{C}+\vec{\omega}_{c} \times \vec{r}_{2}+\vec{V}_{r}
$$

with,

$$
\vec{\omega}_{c}=\left[\omega_{\eta_{1 c}}, \omega_{\zeta_{1 c}}, \omega_{\xi_{1 c}}\right]^{T}, \quad \vec{r}_{2}=\left[s_{2}+u_{2}, v_{2}, w_{2}\right]^{T}, \quad \vec{V}_{r}=\left[\dot{u}_{2}, \dot{v}_{2}, \dot{w}_{2}\right]^{T},
$$

where $\mathrm{T}$ denotes the transpose of the matrix.

Substitution of equations (8a-c), into equation (7), and retaining the third order terms, leads to the following definition of translational velocities for the secondary beam,

$$
\begin{gathered}
V_{x_{2}}=\left\{V_{x_{2}}^{(1)}\right\}+\left\{V_{x_{2}}^{(2)}\right\}=\left\{\dot{v}_{1 c}\right\}+\left\{\dot{u}_{2}+\dot{v}_{1 c}^{\prime} w_{2}-\dot{\varphi}_{1 c} v_{2}+\varphi_{1 c} \dot{w}_{1 c}\right\}, \\
V_{y_{2}}=\left\{V_{y_{2}}^{(1)}\right\}+\left\{V_{y_{2}}^{(2)}\right\}+\left\{V_{y_{2}}^{(3 *)}\right\}=\left\{\dot{v}_{2}+\dot{w}_{1 c}+s_{2} \dot{\varphi}_{1 c}\right\}+\left\{-\varphi_{1 c} \dot{v}_{1 c}+w_{2} \dot{w}_{1 c}^{\prime}+s_{2} \dot{v}_{1 c}^{\prime} w_{1 c}^{\prime}\right\}+ \\
+\left\{u_{2} \dot{\varphi}_{1 c}\right\}, \\
V_{z_{2}}=\left\{V_{z_{2}}^{(1)}\right\}+\left\{V_{z_{2}}^{(2)}\right\}+\left\{V_{z_{2}}^{(3 *)}\right\}=\left\{\dot{w}_{2}-s_{2} \dot{v}_{1, c}^{\prime}\right\}+ \\
+\left\{\dot{u}_{1, c}+v_{1 c}^{\prime} \dot{v}_{1 c}+w_{1 c}^{\prime} \dot{w}_{1 c}-v_{2} \dot{w}_{1 c}^{\prime}-s_{2} \dot{w}_{1 c}^{\prime} \varphi_{1 c}\right\}+\left\{-u_{2} \dot{v}_{1 c}^{\prime}\right\} .
\end{gathered}
$$

Next, it will be applied the following transformations,

$$
W_{2}=w_{2}-s_{2} v_{1 C}^{\prime}+u_{1 C}, V_{2}=v_{2}+s_{2} \varphi_{1 C}+w_{1 C}, \phi_{2}=\varphi_{2}-w_{1 C}^{\prime} .
$$

It is noted that in the case of the linear problem (the first order approximation), the local displacements and rotations of the secondary beam are transformed into global displacements and rotations $\mathrm{W}_{2}, \mathrm{~V}_{2}$, $\phi_{2}$. Therefore, the local displacements of the secondary beam can be replaced by,

$$
\begin{gathered}
w_{2}=W_{2}+s_{2} v_{1 C}^{\prime}-u_{1 C}, \quad \dot{w}_{2}=\dot{W}_{2}+s_{2} \dot{v}_{1 C}^{\prime}-\dot{u}_{1 C}, \quad w_{2}^{\prime}=W_{2}^{\prime}+v_{1 C}^{\prime}, \quad w_{2}^{\prime \prime}=W_{2}^{\prime \prime}, \ldots \text { etc., (11a-d) } \\
v_{2}=V_{2}-s_{2} \varphi_{1 C}-w_{1 C}, \quad \dot{v}_{2}=\dot{V}_{2}-s_{2} \dot{\varphi}_{1 C}-\dot{w}_{1 C}, \quad v_{2}^{\prime}=V_{2}^{\prime}-\varphi_{1 C}, \quad v_{2}^{\prime \prime}=V_{2}^{\prime \prime}, \ldots \text { etc., (12-a-d) } \\
\varphi_{2}=\phi_{2}+w_{1 C}^{\prime}, \quad \dot{\varphi}_{2}=\dot{\phi}_{2}+\dot{w}_{1 C}^{\prime}, \quad \varphi_{2}^{\prime}=\phi_{2}^{\prime}, \ldots \text { etc. }
\end{gathered}
$$

This transformation is essential because it enables a projection of the nonlinear system in the infinite space of the linear normal modes, which were determined by Georgiades et al. (2013b), and then the obtained set of ODEs can be numerically or analytically solved. Considering the transformations given 
in equations (11-13) the angular velocities, curvatures and translational velocities of the secondary beam in the new generalised coordinates are given by equations A.1-2 in Appendix A.

The kinetic and potential energies are given by,

$$
\begin{gathered}
T_{i}=\frac{1}{2} \int_{0}^{l_{i}}\left[m_{i}\left(V_{x_{i}}^{2}+V_{y_{i}}^{2}+V_{z_{i}}^{2}\right)+I_{\xi_{i}} \omega_{\xi_{i}}^{2}+I_{\zeta_{i}} \omega_{\zeta_{i}}^{2}+I_{\eta_{i}} \omega_{\eta_{i}}^{2}\right] d s_{i}=\frac{1}{2} \int_{0}^{l_{i}} h_{T_{i}} d s_{i}, \\
U_{i}=\frac{1}{2} \int_{0}^{l_{i}}\left[D_{\xi_{i}} \rho_{\xi_{i}}^{2}+D_{\eta_{i}} \rho_{\eta_{i}}^{2}+D_{\zeta_{i}} \rho_{\zeta_{i}}^{2}\right] d s_{i}=\frac{1}{2} \int_{0}^{l_{i}} h_{U_{i}} d s_{i},
\end{gathered}
$$

with $\mathrm{i}=1,2$ indicating the primary and secondary beams respectively, and also noting that $m_{i}, I_{\xi_{i}}, I_{\zeta_{i}}$, $I_{\eta_{i}}$ are the inertia coefficient of the beams and $D_{\xi_{i}}, D_{\eta_{i}}, D_{\zeta_{i}}$ are the torsional rigidity and the two bending rigidity stiffnesses coefficients.

In this work it is considered inextensional beams, therefore the following constraints are also imposed,

$$
\begin{gathered}
F_{1}=\frac{1}{2} \int_{0}^{l_{1}} \lambda_{1}\left\{1-\left[\left(1+u_{1}^{\prime}\right)^{2}+\left(w_{1}^{\prime}\right)^{2}+\left(v_{1}^{\prime}\right)^{2}\right]\right\} d s_{1}=\frac{1}{2} \int_{0}^{l_{1}} h_{F_{1}} d s_{1}, \\
F_{2}=\frac{1}{2} \int_{0}^{l_{2}} \lambda_{2}\left\{1-\left[\left(1+u_{2}^{\prime}\right)^{2}+\left(W_{2}^{\prime}+v_{1 C}^{\prime}\right)^{2}+\left(V_{2}^{\prime}-\varphi_{1 C}\right)^{2}\right]\right\} d s_{2}=\frac{1}{2} \int_{0}^{l_{2}} h_{F_{2}} d s_{2} .
\end{gathered}
$$

It should be noted that $\lambda_{1}, \lambda_{2}$ are the Lagrange multipliers for the primary and secondary beam respectively.

It is also considered nonconservative distributed forces $q_{i, j}(i=x, y, z$, and $j=1,2)$ and moments $M_{i, j}$ $(i=x, y, z$, and $j=1,2)$ and also it is considered point loads at the free end of secondary beam with forces $q_{y, p}$ in y-direction (carefully selected to avoid effect in the inextensionality conditions for both beams) $M_{i, p}(i=x, y, z)$. The work done by them, $W_{n c}$, is given by,

$$
\begin{gathered}
W_{n c, 1}=\int_{0}^{l_{1}}\left[q_{x, 1} u_{1}+q_{y, 1} v_{1}+q_{z, 1} w_{1}+M_{x, 1} \varphi_{1}+M_{y, 1} v_{1}^{\prime}-M_{z, 1} w_{1}^{\prime}\right] d s_{1}=\int_{0}^{l_{1}} h_{W_{n c_{1}}} d s_{1}, \\
W_{n c, 2}=\int_{0}^{l_{i}}\left[q_{x, 2} u_{2}+q_{y, 2} v_{2}+q_{z, 2} w_{2}+M_{x, 2} \varphi_{2}+M_{y, 2} v_{2}^{\prime}-M_{z, 2} w_{2}^{\prime}\right] d s_{2}+ \\
+\left[q_{y, p} v_{2}\left(l_{2}, t\right)+M_{x, p} \varphi_{2}\left(l_{2}, t\right)+M_{y, p} v_{2}^{\prime}\left(l_{2}, t\right)-M_{z, p} w_{2}^{\prime}\left(l_{2}, t\right)\right]= \\
=\int_{0}^{l_{2}} h_{W_{n c_{2}}} d s_{2}+h_{W_{n c, p_{2}}}
\end{gathered}
$$

with $i=1,2$ once again, respectively indicating the primary or secondary beams. It should be noted that the nonconservative forces (moments) can be split into external forces (moments) and Rayleigh damping forces (moments). By also considering the transformations of equations (10a-c) and equations (11a), (12a), (13a), then equation (18) for the secondary beam can get the following form,

$$
\begin{gathered}
W_{n c, 2}=\int_{0}^{l_{2}}\left[q_{x, 2} u_{2}+q_{y, 2} V_{2}-s_{2} q_{y, 2} \varphi_{1 C}-q_{y, 2} w_{1 C}+q_{z, 2} W_{2}+s_{2} q_{z, 2} v_{1 C}^{\prime}-q_{z, 2} u_{1 C}+M_{x, 2} \phi_{2}+\right. \\
\left.+M_{x, 2} w_{1 C}^{\prime}+M_{y, 2} V_{2}^{\prime}-M_{y, 2} \varphi_{1 C}-M_{z, 2} W_{2}^{\prime}-M_{z, 2} v_{1 C}^{\prime}\right] d s_{2}+\left[q_{y, p} V_{2}\left(l_{2}, t\right)-l_{2} q_{y, p} \varphi_{1 C}-\right. \\
-q_{y, p} w_{1 C}+M_{x, p} \phi_{2}\left(l_{2}, t\right)+M_{x, p} w_{1 C}^{\prime}+M_{y, p} V_{2}^{\prime}\left(l_{2}, t\right)-M_{y, p} \varphi_{1 C}- \\
\left.-M_{z, p} W_{2}^{\prime}\left(l_{2}, t\right)-M_{z, p} v_{1 C}^{\prime}\right]=\int_{0}^{l_{2}} h_{W_{n c_{2}}} d s_{2}+h_{W_{n c, p}}
\end{gathered}
$$

The linear equations of motion are derived using Hamilton's principle of least action, such that, 


$$
\begin{aligned}
& \delta \int_{t_{1}}^{t_{2}}\left(T_{1}-U_{1}+F_{1}+W_{n c, 1}+T_{2}-U_{2}+F_{2}+W_{n c, 2}\right) d t=0 \Leftrightarrow \\
& \Leftrightarrow \int_{t_{1}}^{t_{2}}\left\{\int_{0}^{l_{1}}\left(\frac{1}{2} \delta h_{T_{1}}-\frac{1}{2} \delta h_{U_{1}}+\frac{1}{2} \delta h_{F_{1}}+\delta h_{W_{n c_{1}}}\right) d s_{1}+\delta h_{W_{n c, p_{1}}}\right. \\
& \left.\quad+\int_{0}^{l_{2}}\left(\frac{1}{2} \delta h_{T_{2}}-\frac{1}{2} \delta h_{U_{2}}+\frac{1}{2} \delta h_{F_{2}}+\delta h_{W_{n c_{2}}}\right) d s_{2}+\delta h_{W_{n c, p_{2}}}\right\} d t=0
\end{aligned}
$$

taking into consideration the right hand sides of equations (14-17).

The variations are defined as follows,

$$
\begin{gathered}
\delta h_{T_{1}}=\sum_{i=1}^{11} \frac{\partial h_{T_{1}}}{\partial p_{i}} \delta p_{i}, \quad p=\left[\dot{u}_{1}, u_{1}^{\prime}, \dot{u}_{1}^{\prime}, \dot{v}_{1}, v_{1}^{\prime}, \dot{v}_{1}^{\prime}, \dot{w}_{1}, \dot{w}_{1}^{\prime}, w_{1}^{\prime}, \dot{\varphi}_{1}, \varphi_{1}\right]^{T}, \\
\delta h_{U_{1}}=\sum_{i=1}^{8} \frac{\partial h_{U_{1}}}{\partial q_{i}} \delta q_{i}, \\
\delta h_{F_{1}}=\sum_{i=1}^{4} \frac{\partial h_{F_{1}}}{\partial r_{i}} \delta r_{i}, \quad q=\left[u_{1}^{\prime}, u_{1}^{\prime \prime}, \varphi_{1}^{\prime}, \varphi_{1}, w_{1}^{\prime \prime}, w_{1}^{\prime}, v_{1}^{\prime \prime}, v_{1}^{\prime}\right]^{T}, \\
\delta h_{T_{2}}=\sum_{i=1}^{27} \frac{\partial h_{T_{2}}}{\partial P_{i}} \delta P_{i}, \quad P=\left[\dot{u}_{2}, u_{2}, \dot{u}_{2}^{\prime}, u_{2}^{\prime}, \dot{V}_{2}, V_{2}, V_{2}^{\prime}, \dot{V}_{2}^{\prime}, \dot{W}_{2}, W_{2}^{\prime}, \dot{W}_{2}^{\prime}, w_{2}^{\prime}, \dot{\phi}_{2}, \phi_{2}, \dot{u}_{1 C}, u_{1 C}, u_{1 C}^{\prime}\right. \\
\left.\dot{u}_{1 C}^{\prime}, \dot{v}_{1 C}, \dot{v}_{1 C}^{\prime}, v_{1 C}^{\prime}, \dot{w}_{1 C}, \dot{w}_{1 C}^{\prime}, w_{1 C}, w_{1 C}^{\prime}, \dot{\varphi}_{1 C}, \varphi_{1 C}\right]^{T} \\
\delta h_{U_{2}}=\sum_{i=1}^{11} \frac{\partial h_{U_{2}}}{\partial Q_{i}} \delta Q_{i}, \quad Q=\left[u_{2}^{\prime}, u_{2}^{\prime \prime}, \phi_{2}^{\prime}, \phi_{2}, W_{2}^{\prime \prime}, W_{2}^{\prime}, V_{2}^{\prime \prime}, V_{2}^{\prime}, v_{1 C}^{\prime}, w_{1 C}^{\prime}, \varphi_{1 C}\right]^{T}, \\
\delta h_{F_{2}}=\sum_{i=1}^{6} \frac{\partial h_{F_{2}}}{\partial R_{i}} \delta R_{i}, \quad R=\left[\lambda_{2}, u_{2}^{\prime}, V_{2}^{\prime}, W_{2}^{\prime}, v_{1 C}^{\prime}, \varphi_{1 C}\right]^{T} \\
=q_{x, 1} \delta u_{1}+q_{y, 1} \delta v_{1}+q_{z, 1} \delta w_{1}+M_{x, 1} \delta \varphi_{1}+M_{y, 1} \delta v_{1}^{\prime}-M_{z, 1} \delta w_{1}^{\prime}, \\
\delta h_{W_{n c_{2}}}=q_{x, 2} \delta u_{2}+q_{y, 2} \delta V_{2}-s_{2} q_{y, 2} \delta \varphi_{1 C}-q_{y, 2} \delta w_{1 C}+q_{z, 2} \delta W_{2}+s_{2} q_{z, 2} \delta v_{1 C}^{\prime}-q_{z, 2} \delta u_{1 C}+ \\
+M_{x, 2} \delta \phi_{2}+M_{x, 2} \delta w_{1 C}^{\prime}+M_{y, 2} \delta V_{2}^{\prime}-M_{y, 2} \delta \varphi_{1 C}-M_{z, 2} \delta W_{2}^{\prime}-M_{z, 2} \delta v_{1 C}^{\prime} . \\
=h_{W_{n c, p_{2}}}=q_{y, p} \delta V_{2}\left(l_{2}, t\right)-l_{2} q_{y, p} \delta \varphi_{1 C}-q_{y, p, 2,2} \delta w_{1 C}+M_{x, p} \delta \phi_{2}\left(l_{2}, t\right)+M_{x, p} \delta w_{1 C}^{\prime}+ \\
+M_{y, p} \delta V_{2}^{\prime}\left(l_{2}, t\right)-M_{y, p} \delta \varphi_{1 C}-M_{z, p} \delta W_{2}^{\prime}\left(l_{2}, t\right)-M_{z, p} \delta v_{1 C}^{\prime}
\end{gathered}
$$

The definitions of curvatures, angular velocities and translational velocities are all given explicitly; by equations 1-3 for the primary beam and by equations A.1-2 in Appendix-A for the secondary beam.

\section{Derivation of Equations}

The equations of motion are derived from Hamilton's principle of least action as stated in equation (20). Considering equations (21a-i) as substitutions within equation (20), and using integration by parts, the following equation emerges,

$$
\begin{gathered}
\int_{t_{1}}^{t_{2}}\left\{\int_{0}^{l_{1}}\left(-\frac{1}{2} \frac{\partial^{2} h_{T_{1}}}{\partial \dot{u}_{1} \partial t}+\frac{1}{2} \frac{\partial^{3} h_{T_{1}}}{\partial \dot{u}_{1}^{\prime} \partial s_{1} \partial t}-\frac{1}{2} \frac{\partial^{2} h_{T_{1}}}{\partial u_{1}^{\prime} \partial s_{1}}-\frac{1}{2} \frac{\partial^{3} h_{U_{1}}}{\partial u_{1}^{\prime \prime} \partial s_{1}^{2}}+\frac{1}{2} \frac{\partial^{2} h_{U_{1}}}{\partial u_{1}^{\prime} \partial s_{1}}-\frac{1}{2} \frac{\partial^{2} h_{F_{1}}}{\partial u_{1}^{\prime} \partial s_{1}}+q_{x, 1}\right) \delta u_{1} d s_{1}+\right. \\
+\left.\left(-\frac{1}{2} \frac{\partial^{2} h_{T_{1}}}{\partial \dot{u}_{1}^{\prime} \partial t}+\frac{1}{2} \frac{\partial h_{T_{1}}}{\partial u_{1}^{\prime}}+\frac{1}{2} \frac{\partial^{2} h_{U_{1}}}{\partial u_{1}^{\prime \prime} \partial s_{1}}-\frac{1}{2} \frac{\partial^{2} h_{U_{1}}}{\partial u_{1}^{\prime}}+\frac{1}{2} \frac{\partial h_{F_{1}}}{\partial u_{1}^{\prime}}\right) \delta u_{1}\right|_{s_{1}=0} ^{s_{1}=l_{1}}-
\end{gathered}
$$




$$
\begin{aligned}
& -\int_{0}^{l_{2}}\left(\frac{1}{2} \frac{\partial^{2} h_{T_{2}}}{\partial \dot{u}_{1 C} \partial t}-\frac{1}{2} \frac{\partial h_{T_{2}}}{\partial u_{1 C}}+q_{z, 2}\right) d s_{2} \delta u_{1 C}-\int_{0}^{l_{1}}\left(\frac{1}{2} \frac{\partial^{2} h_{T_{1}}}{\partial \dot{v}_{1} \partial t}-\frac{1}{2} \frac{\partial^{3} h_{T_{1}}}{\partial \dot{v}_{1}^{\prime} \partial s_{1} \partial t}+\right. \\
& \left.+\frac{1}{2} \frac{\partial^{2} h_{T_{1}}}{\partial v_{1}^{\prime} \partial s_{1}}+\frac{1}{2} \frac{\partial^{2} h_{F_{1}}}{\partial v_{1}^{\prime} \partial s_{1}}+\frac{1}{2} \frac{\partial^{3} h_{U_{1}}}{\partial v_{1}^{\prime \prime} \partial s_{1}^{2}}-\frac{1}{2} \frac{\partial^{2} h_{U_{1}}}{\partial v_{1}^{\prime} \partial s_{1}}-q_{y, 1}+M_{y, 1}^{\prime}\right) \delta v_{1} d s_{1}+ \\
& +\left.\left[\left(-\frac{1}{2} \frac{\partial^{2} h_{T_{1}}}{\partial \dot{v}_{1}^{\prime} \partial t}+\frac{1}{2} \frac{\partial h_{T_{1}}}{\partial v_{1}^{\prime}}+\frac{1}{2} \frac{\partial h_{F_{1}}}{\partial v_{1}^{\prime}}+\frac{1}{2} \frac{\partial^{2} h_{U_{1}}}{\partial v_{1}^{\prime \prime} \partial s_{1}}-\frac{1}{2} \frac{\partial h_{U_{1}}}{\partial v_{1}^{\prime}}+M_{y, 1}\right) \delta v_{1}\right]\right|_{s_{1}=0} ^{s_{1}=l_{1}}- \\
& -\left.\left[\frac{1}{2} \frac{\partial h_{U_{1}}}{\partial v_{1}^{\prime \prime}} \delta v_{1}^{\prime}\right]\right|_{s_{1}=0} ^{s_{1}=l_{1}}-\int_{0}^{l_{2}}\left(\frac{1}{2} \frac{\partial^{2} h_{T_{2}}}{\partial \dot{v}_{1 C} \partial t}\right) d s_{2} \delta v_{1 C}- \\
& -\int_{0}^{l_{2}}\left(\frac{1}{2} \frac{\partial^{2} h_{T_{2}}}{\partial \dot{v}_{1 C}^{\prime} \partial t}-\frac{1}{2} \frac{\partial h_{T_{2}}}{\partial v_{1 C}^{\prime}}-\frac{1}{2} \frac{\partial h_{F_{2}}}{\partial v_{1 C}^{\prime}}+\frac{1}{2} \frac{\partial h_{U_{2}}}{\partial v_{1 C}^{\prime}}-s_{2} q_{z, 2}+M_{z, 2}\right) d s_{2} \delta v_{1 C}^{\prime}- \\
& -\int_{0}^{l_{1}}\left(\frac{1}{2} \frac{\partial^{2} h_{T_{1}}}{\partial \dot{w}_{1} \partial t}-\frac{1}{2} \frac{\partial^{3} h_{T_{1}}}{\partial \dot{w}_{1}^{\prime} \partial s_{1} \partial t}+\frac{1}{2} \frac{\partial^{2} h_{T_{1}}}{\partial w_{1}^{\prime} \partial s_{1}}+\frac{1}{2} \frac{\partial^{2} h_{F_{1}}}{\partial w_{1}^{\prime} \partial s_{1}}+\right. \\
& \left.+\frac{1}{2} \frac{\partial^{3} h_{U_{1}}}{\partial w_{1}^{\prime \prime} \partial s_{1}^{2}}-\frac{1}{2} \frac{\partial^{2} h_{U_{1}}}{\partial w_{1}^{\prime} \partial s_{1}}-q_{z, 1}-M_{z, 1}^{\prime}\right) \delta w_{1} d s_{1}+ \\
& +\left.\left[\left(-\frac{1}{2} \frac{\partial^{2} h_{T_{1}}}{\partial \dot{w}_{1}^{\prime} \partial t}+\frac{1}{2} \frac{\partial h_{T_{1}}}{\partial w_{1}^{\prime}}+\frac{1}{2} \frac{\partial h_{F_{1}}}{\partial w_{1}^{\prime}}+\frac{1}{2} \frac{\partial^{2} h_{U_{1}}}{\partial w_{1}^{\prime \prime} \partial s_{1}}-\frac{1}{2} \frac{\partial h_{U_{1}}}{\partial w_{1}^{\prime}}-M_{z, 1}\right) \delta w_{1}\right]\right|_{s_{1}=0} ^{s_{1}=l_{1}}-\left.\left[\frac{1}{2} \frac{\partial h_{U_{1}}}{\partial w_{1}^{\prime \prime}} \delta w_{1}^{\prime}\right]\right|_{S_{1}=0} ^{s_{1}=l_{1}}- \\
& -\int_{0}^{l_{2}}\left(\frac{1}{2} \frac{\partial^{2} h_{T_{2}}}{\partial \dot{w}_{1 C} \partial t}-\frac{1}{2} \frac{\partial h_{T_{2}}}{\partial w_{1 C}}+q_{y, 2}\right) d s_{2} \delta w_{1 C}-\int_{0}^{l_{2}}\left(\frac{1}{2} \frac{\partial^{2} h_{T_{2}}}{\partial \dot{w}_{1 C}^{\prime} \partial t}-\frac{1}{2} \frac{\partial h_{T_{2}}}{\partial w_{1 C}^{\prime}}+\frac{1}{2} \frac{\partial h_{U_{2}}}{\partial w_{1 C}^{\prime}}-M_{x, 2}\right) d s_{2} \delta w_{1 C}^{\prime}- \\
& -\int_{0}^{l_{1}}\left(\frac{1}{2} \frac{\partial^{2} h_{T_{1}}}{\partial \dot{\varphi}_{1} \partial t}-\frac{1}{2} \frac{\partial h_{T_{1}}}{\partial \varphi_{1}}-\frac{1}{2} \frac{\partial^{2} h_{U_{1}}}{\partial \varphi_{1}^{\prime} \partial s_{1}}+\frac{1}{2} \frac{\partial h_{U_{1}}}{\partial \varphi_{1}}-M_{x, 1}\right) \delta \varphi_{1} d s_{1}-\left.\left(\frac{1}{2} \frac{\partial h_{U_{1}}}{\partial \varphi_{1}^{\prime}} \delta \varphi_{1}\right)\right|_{s_{1}=0} ^{s_{1}=l_{1}}- \\
& -\int_{0}^{l_{2}}\left(\frac{1}{2} \frac{\partial^{2} h_{T_{2}}}{\partial \dot{\varphi}_{1 C} \partial t}-\frac{1}{2} \frac{\partial h_{T_{2}}}{\partial \varphi_{1 C}}-\frac{1}{2} \frac{\partial h_{F_{2}}}{\partial \varphi_{1 C}}+\frac{1}{2} \frac{\partial h_{U_{2}}}{\partial \varphi_{1 C}}+s_{2} q_{y, 2}+M_{y, 2}\right) d s_{2} \delta \varphi_{1 C}+\int_{0}^{l_{1}} \frac{1}{2} \frac{\partial h_{F_{1}}}{\partial \lambda_{1}} \delta \lambda_{1} d s_{1}- \\
& -\int_{0}^{l_{2}}\left(\frac{1}{2} \frac{\partial^{2} h_{T_{2}}}{\partial \dot{u}_{2} \partial t}-\frac{1}{2} \frac{\partial^{3} h_{T_{2}}}{\partial \dot{u}_{2}^{\prime} \partial s_{2} \partial t}+\frac{1}{2} \frac{\partial^{2} h_{T_{2}}}{\partial u_{2}^{\prime} \partial s_{2}}-\frac{1}{2} \frac{\partial h_{T_{2}}}{\partial u_{2}}+\frac{1}{2} \frac{\partial^{3} h_{U_{2}}}{\partial u_{2}^{\prime \prime} \partial s_{2}^{2}}-\frac{1}{2} \frac{\partial^{2} h_{U_{2}}}{\partial u_{2}^{\prime} \partial s_{2}}+\frac{1}{2} \frac{\partial^{2} h_{F_{2}}}{\partial u_{2}^{\prime} \partial s_{2}}-q_{x, 2}\right) \delta u_{2} d s_{2}+ \\
& +\left.\left(\frac{1}{2} \frac{\partial^{2} h_{T_{2}}}{\partial \dot{u}_{2}^{\prime} \partial t}-\frac{1}{2} \frac{\partial h_{T_{2}}}{\partial u_{2}^{\prime}}-\frac{1}{2} \frac{\partial^{2} h_{U_{2}}}{\partial u_{2}^{\prime \prime} \partial s_{2}}+\frac{1}{2} \frac{\partial h_{U_{2}}}{\partial u_{2}^{\prime}}+\frac{1}{2} \frac{\partial h_{F_{2}}}{\partial u_{2}^{\prime}}\right) \delta u_{2}\right|_{S_{2}=0} ^{s_{2}=l_{2}}- \\
& -\int_{0}^{l_{2}}\left(\frac{1}{2} \frac{\partial^{2} h_{T_{2}}}{\partial \dot{V}_{2} \partial t}-\frac{1}{2} \frac{\partial^{3} h_{T_{2}}}{\partial \dot{V}_{2}^{\prime} \partial s_{2} \partial t}+\frac{1}{2} \frac{\partial^{2} h_{T_{2}}}{\partial V_{2}^{\prime} \partial s_{2}}-\frac{1}{2} \frac{\partial h_{T_{2}}}{\partial V_{2}}+\right. \\
& \left.+\frac{1}{2} \frac{\partial^{2} h_{F_{2}}}{\partial V_{2}^{\prime} \partial s_{2}}+\frac{1}{2} \frac{\partial^{3} h_{U_{2}}}{\partial V_{2}^{\prime \prime} \partial s_{2}^{2}}-\frac{1}{2} \frac{\partial^{2} h_{U_{2}}}{\partial V_{2}^{\prime} \partial s_{2}}-q_{y, 2}+M_{y, 2}^{\prime}\right) \delta V_{2} d s_{2}-\left.\left[\frac{1}{2} \frac{\partial h_{U_{2}}}{\partial V_{2}^{\prime \prime}} \delta V_{2}^{\prime}\right]\right|_{s_{2}=0} ^{s_{2}=l_{2}}+ \\
& +\left.\left[\left(-\frac{1}{2} \frac{\partial^{2} h_{T_{2}}}{\partial \dot{V}_{2}^{\prime} \partial t}+\frac{1}{2} \frac{\partial h_{T_{2}}}{\partial V_{2}^{\prime}}+\frac{1}{2} \frac{\partial h_{F_{2}}}{\partial V_{2}^{\prime}}+\frac{1}{2} \frac{\partial^{2} h_{U_{2}}}{\partial V_{2}^{\prime \prime} \partial s_{2}}-\frac{1}{2} \frac{\partial h_{U_{2}}}{\partial V_{2}^{\prime}}+M_{y, 2}\right) \delta V_{2}\right]\right|_{S_{2}=0} ^{s_{2}=l_{2}}- \\
& -\int_{0}^{l_{2}}\left(\frac{1}{2} \frac{\partial^{2} h_{T_{2}}}{\partial \dot{W}_{2} \partial t}-\frac{1}{2} \frac{\partial^{3} h_{T_{2}}}{\partial \dot{W}_{2}^{\prime} \partial s_{2} \partial t}-\frac{1}{2} \frac{\partial h_{T_{2}}}{\partial W_{2}}+\frac{1}{2} \frac{\partial^{2} h_{T_{2}}}{\partial W_{2}^{\prime} \partial s_{2}}+\frac{1}{2} \frac{\partial^{2} h_{F_{2}}}{\partial W_{2}^{\prime} \partial s_{2}}+\right. \\
& \left.+\frac{1}{2} \frac{\partial^{3} h_{U_{2}}}{\partial W_{2}^{\prime \prime} \partial s_{2}^{2}}-\frac{1}{2} \frac{\partial^{2} h_{U_{2}}}{\partial W_{2}^{\prime} \partial s_{2}}-q_{z, 2}-M_{z, 2}^{\prime}\right) \delta W_{2} d s_{2}-
\end{aligned}
$$




$$
\begin{aligned}
& \left.-\left[\frac{1}{2} \frac{\partial h_{U_{2}}}{\partial W_{2}^{\prime \prime}} \delta W_{2}^{\prime}\right]\right]\left.\right|_{S_{2}=0} ^{S_{2}=l_{2}}+\left.\left[\left(-\frac{1}{2} \frac{\partial^{2} h_{T_{2}}}{\partial \dot{W}_{2}^{\prime} \partial t}+\frac{1}{2} \frac{\partial h_{T_{2}}}{\partial W_{2}^{\prime}}+\frac{1}{2} \frac{\partial h_{F_{2}}}{\partial W_{2}^{\prime}}+\frac{1}{2} \frac{\partial^{2} h_{U_{2}}}{\partial W_{2}^{\prime \prime} \partial s_{2}}-\frac{1}{2} \frac{\partial h_{U_{2}}}{\partial W_{2}^{\prime}}-M_{z, 2}\right) \delta W_{2}\right]\right|_{S_{2}=0} ^{s_{2}=l_{2}}- \\
& -\int_{0}^{l_{2}}\left(\frac{1}{2} \frac{\partial^{2} h_{T_{2}}}{\partial \dot{\phi}_{2} \partial t}-\frac{1}{2} \frac{\partial h_{T_{2}}}{\partial \phi_{2}}-\frac{1}{2} \frac{\partial^{2} h_{U_{2}}}{\partial \phi_{2}^{\prime} \partial s_{2}}+\frac{1}{2} \frac{\partial h_{U_{2}}}{\partial \phi_{2}}-M_{x, 2}\right) \delta \phi_{2} d s_{2}- \\
& -\left.\left[\frac{1}{2} \frac{\partial h_{U_{2}}}{\partial \phi_{2}^{\prime}} \delta \phi_{2}\right]\right|_{S_{2}=0} ^{s_{2}=l_{2}}+\int_{0}^{l_{2}} \frac{1}{2} \frac{\partial h_{F_{2}}}{\partial \lambda_{2}} \delta \lambda_{2} d s_{2}+\left[q_{y, p} \delta V_{2}\left(l_{2}, t\right)-l_{2} q_{y, p} \delta \varphi_{1 C}-q_{y, p} \delta w_{1 C}+\right. \\
& \left.\left.+M_{x, p} \delta \phi_{2}\left(l_{2}, t\right)+M_{x, p} \delta w_{1 C}^{\prime}+M_{y, p} \delta V_{2}^{\prime}\left(l_{2}, t\right)-M_{y, p} \delta \varphi_{1 C}-M_{z, p} \delta W_{2}^{\prime}\left(l_{2}, t\right)-M_{z, p} \delta v_{1 C}^{\prime}\right]\right\} d t=0 .
\end{aligned}
$$

It should be mentioned that spatial variations with respect to the derivative (in space) of axial motion $\delta u_{1 C}^{\prime}, \delta \dot{u}_{1 C}^{\prime}$ have been neglected since they would lead to additional boundary conditions involving $u_{1 C}^{\prime}$. The axial equation of motion is second order therefore the solution of the boundary value problem can be solved using only the boundary conditions of the axial motion $\left.u_{1}\right|_{s_{1}=0} ^{s_{1}=l_{1}}$ and neglecting those involving the derivatives e.g. $u_{1 C}^{\prime}$, otherwise the problem will be over-determined.

At this stage it is separated the non-conservative forces and moments, splitting them into external forces (moments) and Rayleigh damping forces (moments), with damping coefficients $c_{i j}$ having $i=1$ for the primary beam and $i=2$ for the secondary beam. Taking local velocities then the non-conservative forces are taking the following explicit form (Nayfeh and Pai 2004):

-Axial motion of the primary beam $\left(u_{1}\right)$

In order to be consistent with the inextensionality condition, the external forces should be zero and the Rayleigh damping term has the form:

$$
q_{x, 1}=-c_{11} \dot{u}_{1}
$$

-In-plane bending motion of the primary beam $\left(v_{1}\right)$,

$$
q_{y, 1}=\tilde{q}_{y, 1}-c_{12} \dot{v}_{1}
$$

-Out-of-plane bending motion of the primary beam $\left(w_{1}\right)$,

$$
q_{z, 1}=\tilde{q}_{z, 1}-c_{13} \dot{w}_{1}
$$

-Torsional motion of the primary beam $\left(\varphi_{1}\right)$,

$$
M_{x, 1}=\widetilde{M}_{x, 1}-c_{14} \dot{\varphi}_{1}
$$

-Axial motion of the secondary beam $\left(u_{2}\right)$,

Similarly to the primary beam, in order to be consistent with the inextensionality condition, the external forces should be zero and the Rayleigh damping term is as follows:

$$
q_{x, 2}=-c_{21} \dot{u}_{2}
$$

-In-plane bending motion of the secondary beam $\left(W_{2}\right)$,

$$
q_{z, 2}=\tilde{q}_{z, 2}-c_{22} \dot{w}_{2}=\tilde{q}_{z, 2}-c_{22} \dot{W}_{2}-c_{22} s_{2} \dot{v}_{1 C}^{\prime}+c_{22} \dot{u}_{1 C},
$$

expressed in global velocities using the transformation from equation (11.b).

-Out-of-plane bending motion of the primary beam $\left(V_{2}\right)$,

$$
q_{y, 2}=\tilde{q}_{y, 2}-c_{23} \dot{v}_{2}=\tilde{q}_{y, 2}-c_{23} \dot{V}_{2}+c_{23} s_{2} \dot{\varphi}_{1 C}+c_{23} \dot{w}_{1 C},
$$


expressed in global velocities using the transformation from equation (12.b).

-Torsional motion of the primary beam $\left(\phi_{2}\right)$,

$$
M_{x, 2}=\widetilde{M}_{x, 2}-c_{24} \dot{\varphi}_{2}=\widetilde{M}_{x, 2}-c_{24} \dot{\phi}_{2}-c_{24} \dot{w}_{1 C}^{\prime},
$$

expressed in global velocities using the transformation from equation (13.b).

Evaluation of the partial derivatives of equation (22), and then using firstly equations (B.1a-b) and also equation (23a) leads to the following equation for axial motion $\left(u_{1}\right)$ of the primary beam:

$$
\begin{aligned}
& m_{1} \ddot{u}_{1}+ \frac{\partial}{\partial s_{1}}\left[I_{\zeta_{1}} \delta_{3} v_{1}^{\prime} \ddot{v}_{1}^{\prime}\right]+\frac{\partial}{\partial s_{1}}\left[I_{\eta_{1}} \delta_{1} w_{1}^{\prime} \ddot{w}_{1}^{\prime}\right]-\frac{\partial^{2}}{\partial s_{1}^{2}}\left[D_{\eta_{1}} w_{1}^{\prime} w_{1}^{\prime \prime}\right]-\frac{\partial^{2}}{\partial s_{1}^{2}}\left[D_{\zeta_{1}} v_{1}^{\prime} v_{1}^{\prime \prime}\right]+ \\
&+\frac{\partial}{\partial s_{1}}\left[D_{\eta_{1}} w_{1}^{\prime \prime 2}\right]+\frac{\partial}{\partial s_{1}}\left[D_{\zeta_{1}} v_{1}^{\prime \prime 2}\right]-\frac{\partial}{\partial s_{1}}\left[\lambda_{1}\left(1+u_{1}^{\prime}\right)\right]+c_{11} \dot{u}_{1}=0
\end{aligned}
$$

and then secondly equations (B.2a-f) with equation (23b) will lead to this equation for in-plane bending motion $\left(v_{1}\right)$ of the primary beam:

$$
\begin{gathered}
m_{1} \ddot{v}_{1}-\frac{\partial}{\partial s_{1}}\left[I_{\xi_{1}}\left(\dot{w}_{1}^{\prime} \dot{\varphi}_{1}+w_{1}^{\prime} \ddot{\varphi}_{1}\right)\right]+\frac{\partial}{\partial s_{1}}\left[\delta_{1} I_{\eta_{1}}\left(\dot{\varphi}_{1} \dot{w}_{1}^{\prime}+\varphi_{1} \ddot{w}_{1}^{\prime}\right)\right]- \\
-\frac{\partial}{\partial s_{1}}\left[I_{\zeta_{1}} \delta_{3}\left(\ddot{v}_{1}^{\prime}+\ddot{w}_{1}^{\prime} \varphi_{1}+\dot{\varphi}_{1} \dot{w}_{1}^{\prime}\right)\right]-\frac{\partial}{\partial s_{1}}\left[\lambda_{1} v_{1}^{\prime}\right]+\frac{\partial^{2}}{\partial s_{1}^{2}}\left[D_{\xi_{1}} w_{1}^{\prime} \varphi_{1}^{\prime}\right]- \\
-\frac{\partial^{2}}{\partial s_{1}^{2}}\left[D_{\eta_{1}} \varphi_{1} w_{1}^{\prime \prime}\right]+\frac{\partial^{2}}{\partial s_{1}^{2}}\left[D_{\zeta_{1}}\left(v_{1}^{\prime \prime}+\varphi_{1} w_{1}^{\prime \prime}\right)\right]-\tilde{q}_{y, 1}+c_{12} \dot{v}_{1}+\frac{\partial M_{y, 1}}{\partial s_{1}}=0,
\end{gathered}
$$

after which the next equations (B.3a-f) with equation (23c) will generate the equation for the out-ofplane bending motion $\left(w_{1}\right)$ of the primary beam:

$$
\begin{aligned}
m_{1} \ddot{w}_{1}+ & \frac{\partial}{\partial s_{1}}\left[I_{\eta_{1}} \delta_{1}\left(-\ddot{w}_{1}^{\prime}+\dot{\varphi}_{1} \dot{v}_{1}^{\prime}+\varphi_{1} \ddot{v}_{1}^{\prime}\right)\right]-\frac{\partial}{\partial s_{1}}\left[I_{\zeta_{1}} \delta_{3}\left(\dot{\varphi}_{1} \dot{v}_{1}^{\prime}+\varphi_{1} \ddot{v}_{1}^{\prime}\right)\right]+ \\
+ & \frac{\partial}{\partial s_{1}}\left[I_{\xi_{1}} \dot{v}_{1}^{\prime} \dot{\varphi}_{1}\right]-\frac{\partial}{\partial s_{1}}\left[\lambda_{1} w_{1}^{\prime}\right]-\frac{\partial^{2}}{\partial s_{1}^{2}}\left[D_{\eta_{1}}\left(-w_{1}^{\prime \prime}+\varphi_{1} v_{1}^{\prime \prime}\right)\right]+ \\
+ & \frac{\partial^{2}}{\partial s_{1}^{2}}\left[D_{\zeta_{1}} \varphi_{1} v_{1}^{\prime \prime}\right]-\frac{\partial}{\partial s_{1}}\left[D_{\xi_{1}} v_{1}^{\prime \prime} \varphi_{1}^{\prime}\right]-\tilde{q}_{z, 1}+c_{13} \dot{w}_{1}-\frac{\partial M_{z, 1}}{\partial s_{1}}=0,
\end{aligned}
$$

and then equations (B.4a-d) with equation (23d) render the next equation for torsional motion $\left(\varphi_{1}\right)$ of the primary beam:

$$
\begin{gathered}
I_{\xi_{1}}\left(\ddot{\varphi}_{1}+\ddot{v}_{1}^{\prime} w_{1}^{\prime}+\dot{v}_{1}^{\prime} \dot{w}_{1}^{\prime}\right)+I_{\eta_{1}} \delta_{1} \dot{v}_{1}^{\prime} \dot{w}_{1}^{\prime}-I_{\zeta_{1}} \delta_{3} \dot{v}_{1}^{\prime} \dot{w}_{1}^{\prime}-\frac{\partial}{\partial s_{1}}\left[D_{\xi_{1}}\left(\varphi_{1}^{\prime}+v_{1}^{\prime \prime} w_{1}^{\prime}\right)\right]- \\
-D_{\eta_{1}} v_{1}^{\prime \prime} w_{1}^{\prime \prime}+D_{\zeta_{1}} v_{1}^{\prime \prime} w_{1}^{\prime \prime}-\widetilde{M}_{x, 1}+c_{14} \dot{\varphi}_{1}=0 .
\end{gathered}
$$

Furthermore, equations (B.5a-c) with equation (23e) provide an equation for axial motion $\left(u_{2}\right)$ of the secondary beam:

$$
m_{2}\left(\ddot{v}_{1 c}+\ddot{u}_{2}+\ddot{v}_{1 c}^{\prime} W_{2}+2 \dot{v}_{1 c}^{\prime} \dot{W}_{2}+s_{2} \ddot{v}_{1 c}^{\prime} v_{1 c}^{\prime}+s_{2} \dot{v}_{1 C}^{\prime 2}-\right.
$$




$$
\begin{gathered}
\left.-\ddot{\varphi}_{1 c} V_{2}-2 \dot{\varphi}_{1 c} \dot{V}_{2}+s_{2} \dot{\varphi}_{1 c}^{2}+s_{2} \varphi_{1 c} \ddot{\varphi}_{1 c}+\dot{\varphi}_{1 c} \dot{W}_{1 c}+\varphi_{1 c} \ddot{W}_{1 c}+\ddot{\varphi}_{1 c} W_{1 c}+\dot{\varphi}_{1 c} \dot{W}_{1 c}\right)- \\
-\frac{\partial}{\partial s_{2}}\left[\lambda_{2}\left(1+u_{2}^{\prime}\right)\right]+c_{21} \dot{u}_{2}-\frac{\partial^{2}}{\partial s_{2}^{2}}\left[D_{\eta_{2}}\left(W_{2}^{\prime} W_{2}^{\prime \prime}+v_{1 c}^{\prime} W_{2}^{\prime \prime}\right)\right]+\frac{\partial^{2}}{\partial s_{2}^{2}}\left[D_{\zeta_{2}}\left(-V_{2}^{\prime} V_{2}^{\prime \prime}+\varphi_{1 c} V_{2}^{\prime \prime}\right)\right]+ \\
+\frac{\partial}{\partial s_{2}}\left[D_{\eta_{2}} W_{2}^{\prime \prime 2}\right]+\frac{\partial}{\partial s_{2}}\left[D_{\zeta_{2}} V_{2}^{\prime \prime 2}\right]- \\
-\frac{\partial}{\partial s_{2}}\left[I_{\eta_{2}} \delta_{3}\left(-W_{2}^{\prime} \ddot{W}_{2}^{\prime}+v_{1 c} \ddot{W}_{2}^{\prime}\right)\right]-\frac{\partial}{\partial s_{2}}\left[I_{\zeta_{2}} \delta_{2}\left(-\ddot{V}_{2}^{\prime} V_{2}^{\prime}+\varphi_{1 c} \ddot{V}_{2}^{\prime}\right)\right]=0 .
\end{gathered}
$$

From equations (B.6a-g) with equation (23f) one finds the equation for in-plane bending motion $\left(W_{2}\right)$ of the secondary beam:

$$
\begin{gathered}
m_{2}\left(\ddot{W}_{2}+v_{1 c}^{\prime} \ddot{v}_{1 c}+\dot{w}_{1 c}^{\prime} \dot{w}_{1 c}+w_{1 c}^{\prime} \ddot{w}_{1 c}-2 \dot{V}_{2} \dot{w}_{1 c}^{\prime}-V_{2} \ddot{w}_{1 c}^{\prime}+\dot{w}_{1 c} \dot{w}_{1 c}^{\prime}+w_{1 c} \ddot{w}_{1 c}^{\prime}\right)- \\
-\frac{\partial}{\partial s_{2}}\left[I_{\zeta_{2}} \delta_{2}\left(\dot{\phi}_{2} \dot{V}_{2}^{\prime}+\phi_{2} \ddot{V}_{2}^{\prime}+\dot{w}_{1 c}^{\prime} \dot{V}_{2}^{\prime}+w_{1 c}^{\prime} \ddot{V}_{2}^{\prime}\right)\right]+\frac{\partial}{\partial s_{2}}\left[I _ { \eta _ { 2 } } \delta _ { 3 } \left(-\ddot{W}_{2}^{\prime}+\dot{\phi}_{2} \dot{V}_{2}^{\prime}+\phi_{2} \ddot{V}_{2}^{\prime}+\dot{w}_{1 c}^{\prime} \dot{V}_{2}^{\prime}+\right.\right. \\
\left.\left.+w_{1 c}^{\prime} \ddot{V}_{2}^{\prime}-\dot{\phi}_{2} \dot{\varphi}_{1 c}-\phi_{2} \ddot{\varphi}_{1 c}-\dot{w}_{1 c}^{\prime} \dot{\varphi}_{1 c}-w_{1 c}^{\prime} \ddot{\varphi}_{1 c}+\ddot{w}_{1 c}^{\prime} \varphi_{1 c}+\dot{w}_{1 c}^{\prime} \dot{\varphi}_{1 c}\right)\right]+ \\
+\frac{\partial}{\partial s_{2}}\left[I_{\xi_{2}}\left(\dot{V}_{2}^{\prime} \dot{\phi}_{2}-\dot{\varphi}_{1 c} \dot{\phi}_{2}\right)\right]- \\
-\frac{\partial}{\partial s_{2}}\left[\lambda_{2}\left(W_{2}^{\prime}+v_{1 C}^{\prime}\right)\right]-\frac{\partial^{2}}{\partial s_{2}^{2}}\left[D_{\eta_{2}}\left(-W_{2}^{\prime \prime}+\phi_{2} V_{2}^{\prime \prime}+w_{1 c}^{\prime} V_{2}^{\prime \prime}\right)\right]+\frac{\partial^{2}}{\partial s_{2}^{2}}\left[D_{\zeta_{2}}\left(V_{2}^{\prime \prime} \phi_{2}+V_{2}^{\prime \prime} w_{1 C}^{\prime}\right)\right]- \\
-\frac{\partial}{\partial s_{2}}\left[D_{\xi_{2}} \phi_{2}^{\prime} V_{2}^{\prime \prime}\right]-\tilde{q}_{z, 2}+c_{22} \dot{W}_{2}+c_{22} s_{2} \dot{v}_{1 C}^{\prime}-c_{22} \dot{u}_{1 C}-\frac{\partial M_{z, 2}}{\partial s_{2}}=0,
\end{gathered}
$$

penultimately equations (B.7a-g) produce the next equation for the out-of-plane bending motion $\left(V_{2}\right)$ of the secondary beam:

$$
\begin{gathered}
m_{2}\left(\ddot{V}_{2}-\varphi_{1 c} \ddot{v}_{1 c}+2 \dot{W}_{2} \dot{w}_{1 c}^{\prime}+W_{2} \ddot{w}_{1 c}^{\prime}+s_{2} \ddot{v}_{1 c}^{\prime} w_{1 c}^{\prime}+s_{2} \dot{v}_{1 c}^{\prime} \dot{w}_{1 c}^{\prime}+s_{2} \dot{v}_{1 c}^{\prime} \dot{w}_{1 c}^{\prime}+s_{2} v_{1 c}^{\prime} \ddot{w}_{1 c}^{\prime}\right)- \\
-\frac{\partial}{\partial s_{2}}\left[I_{\xi_{2}}\left(\dot{W}_{2}^{\prime} \dot{\phi}_{2}+W_{2}^{\prime} \ddot{\phi}_{2}+\dot{v}_{1 c}^{\prime} \dot{\phi}_{2}+v_{1 c}^{\prime} \ddot{\phi}_{2}\right)\right]- \\
-\frac{\partial}{\partial s_{2}}\left[I_{\zeta_{2}} \delta_{2}\left(\ddot{V}_{2}^{\prime}+\ddot{W}_{2}^{\prime} \phi_{2}+\dot{\phi}_{2} \dot{W}_{2}^{\prime}+\ddot{v}_{1 c}^{\prime} \phi_{2}+\dot{v}_{1 c}^{\prime} \dot{\phi}_{2}+\ddot{W}_{2}^{\prime} w_{1 c}^{\prime}+\dot{W}_{2}^{\prime} \dot{w}_{1 c}^{\prime}+2 \ddot{v}_{1 c}^{\prime} w_{1 c}^{\prime}+2 \dot{v}_{1 c}^{\prime} \dot{w}_{1 c}^{\prime}\right)\right]+ \\
+\frac{\partial}{\partial s_{2}}\left[I_{\eta_{2}} \delta_{3}\left(\dot{\phi}_{2} \dot{W}_{2}^{\prime}+\phi_{2} \ddot{W}_{2}^{\prime}+\dot{w}_{1 c}^{\prime} \dot{W}_{2}^{\prime}+w_{1 c}^{\prime} \ddot{W}_{2}^{\prime}\right)\right]-\frac{\partial}{\partial s_{2}}\left[\lambda_{2}\left(V_{2}^{\prime}-\varphi_{1 C}\right)\right]+ \\
+\frac{\partial^{2}}{\partial s_{2}^{2}}\left[D_{\xi_{2}}\left(W_{2}^{\prime} \phi_{2}^{\prime}+v_{1 c}^{\prime} \phi_{2}^{\prime}\right)\right]-\frac{\partial^{2}}{\partial s_{2}^{2}}\left[D_{\eta_{2}}\left(\phi_{2} W_{2}^{\prime \prime}+w_{1 C}^{\prime} W_{2}^{\prime \prime}\right)\right]+ \\
+\frac{\partial^{2}}{\partial s_{2}^{2}}\left[D_{\zeta_{2}}\left(V_{2}^{\prime \prime}+\phi_{2} W_{2}^{\prime \prime}+w_{1 C}^{\prime} W_{2}^{\prime \prime}\right)\right]-\tilde{q}_{y, 2}+c_{23} \dot{V}_{2}-c_{23} s_{2} \dot{\varphi}_{1 C}-c_{23} \dot{w}_{1 C}+\frac{\partial M_{y, 2}}{\partial s_{2}}=0 .
\end{gathered}
$$

Finally, equations (B.8a-d) give rise to the equation for torsional motion $\left(\phi_{2}\right)$ of the secondary beam:

$$
\begin{gathered}
I_{\xi_{2}}\left(\ddot{\phi}_{2}+\ddot{V}_{2}^{\prime} W_{2}^{\prime}+\dot{V}_{2}^{\prime} \dot{W}_{2}^{\prime}+\ddot{V}_{2}^{\prime} v_{1 C}^{\prime}+\dot{V}_{2}^{\prime} \dot{v}_{1 C}^{\prime}-\ddot{\varphi}_{1 c} W_{2}^{\prime}-\dot{\varphi}_{1 c} \dot{W}_{2}^{\prime}-\ddot{\varphi}_{1 c} v_{1 C}^{\prime}-\dot{\varphi}_{1 c} \dot{v}_{1 C}^{\prime}+\right. \\
\left.+\delta_{1} \dot{\varphi}_{1 c} \dot{v}_{1 c}^{\prime}+\delta_{1} \varphi_{1 c} \ddot{v}_{1 c}^{\prime}\right)-I_{\eta_{2}} \delta_{3}\left(-\dot{V}_{2}^{\prime} \dot{W}_{2}^{\prime}+\dot{\varphi}_{1 c} \dot{W}_{2}^{\prime}\right)-I_{\zeta_{2}} \delta_{2}\left(\dot{V}_{2}^{\prime} \dot{W}_{2}^{\prime}+\dot{V}_{2}^{\prime} \dot{v}_{1 c}^{\prime}\right)-D_{\eta_{2}} W_{2}^{\prime \prime} V_{2}^{\prime \prime}- \\
-\frac{\partial}{\partial s_{2}}\left[D_{\xi_{2}}\left(\phi_{2}^{\prime}+V_{2}^{\prime \prime} W_{2}^{\prime}+v_{1 C}^{\prime} V_{2}^{\prime \prime}\right)\right]+D_{\zeta_{2}} W_{2}^{\prime \prime} V_{2}^{\prime \prime}-\widetilde{M}_{x, 2}+c_{24} \dot{\phi}_{2}+c_{24} \dot{W}_{1 C}^{\prime}=0 .
\end{gathered}
$$


There are two kinds of boundary condition applied in this problem and these are known, respectively, as strong and weak. The former arise from the system configuration and are in the form of restrictions to motion in both displacement and rotation. The latter boundary conditions arise from force and moment equillibria at the interconnections of the two principal substructures which constitute the overall system, and can be obtained explicitly through equation (22), which arise from the Extended Hamilton Principle.

The strong boundary conditions arising from the system configuration, namely the clamped ends shown in Fig. 1, are, for the primary beam:

a) Axial motion,

b) In-plane bending,

$$
u_{1}(0, t)=0 .
$$

$$
v_{1}(0, t)=0, \quad v_{1}^{\prime}(0, t)=0 .
$$

c) Out-of-plane bending,

$$
w_{1}(0, t)=0, \quad w_{1}^{\prime}(0, t)=0 .
$$

d) Torsional motion,

$$
\varphi_{1}(0, t)=0
$$

In the case of the secondary beam they are:

a) Axial motion,

b) In-plane bending,

c) Out-of-plane bending,

$$
w_{2}(0, t)=0, \quad w_{2}^{\prime}(0, t)=0 .
$$

d) Torsional motion,

$$
v_{2}(0, t)=0, \quad v_{2}^{\prime}(0, t)=0 .
$$

$$
\varphi_{2}(0, t)=0 .
$$

Using the transformations given by equations (10)-(13) the strong boundary conditions (equations 3639) for the secondary beam are seen to take these forms,
a) Axial motion,
b) In-plane bending,

$$
u_{2}(0, t)=0
$$
c) Out-of-plane bending,

$$
W_{2}(0, t)=u_{1 C}, \quad W_{2}^{\prime}(0, t)=-v_{1 C}^{\prime} .
$$
d) Torsional motion,

$$
V_{2}(0, t)=w_{1 C}, \quad V_{2}^{\prime}(0, t)=\varphi_{1 C}
$$

$$
\phi_{2}(0, t)=-w_{1 C}^{\prime}
$$

Considering now the variations of the strong boundary conditions of the primary beam (equations 3235 ) and of the secondary beam (equations 40-43), the variations in the boundary conditions are as follows:

-Primary beam,

a) Axial motion,

b) In-plane bending,

$$
\delta u_{1}(0, t)=0 .
$$

$$
\delta v_{1}(0, t)=0, \quad \delta v_{1}^{\prime}(0, t)=0 .
$$


c) Out-of-plane bending,

$$
\delta w_{1}(0, t)=0, \quad \delta w_{1}^{\prime}(0, t)=0 .
$$

d) Torsional motion,

$$
\delta \varphi_{1}(0, t)=0 .
$$

-Secondary beam,

a) Axial motion,

b) In-plane bending,

$$
\delta u_{2}(0, t)=0 .
$$

c) Out-of-plane bending,

$$
\delta W_{2}(0, t)=\delta u_{1 C}, \quad \delta W_{2}^{\prime}(0, t)=-\delta v_{1 C}^{\prime} .
$$

d) Torsional motion,

$$
\delta V_{2}(0, t)=\delta w_{1 C}, \quad \delta V_{2}^{\prime}(0, t)=\delta \varphi_{1 C} .
$$

$$
\delta \phi_{2}(0, t)=-\delta w_{1 C}^{\prime} .
$$

The weak boundary conditions for the primary beam at point $\mathrm{C}$ for which $s_{1}=l_{1}$ and for the secondary beam at $s_{2}=l_{2}$ are represented through Hamilton's equation (22) by defining the individual terms (noting that these are shown in detail in App. B) and summing them together, all as summarised in the following statement:

-Primary beam,

a) Axial motion, by summing the terms from equations (B.9a-c) which correspond to $\delta u_{1 C}$ using also equation (23f) and then by setting them equal to zero,

$$
\begin{gathered}
{\left.\left[I_{\zeta_{1}} \delta_{3} v_{1}^{\prime} \ddot{v}_{1}^{\prime}\right]\right|_{s_{1}=l_{1}}+\left.\left[I_{\eta_{1}} \delta_{1} w_{1}^{\prime} \ddot{w}_{1}^{\prime}\right]\right|_{s_{1}=l_{1}}-\left.\left[\frac{\partial}{\partial s_{1}}\left(D_{\eta_{1}} w_{1}^{\prime} w_{1}^{\prime \prime}\right)\right]\right|_{s_{1}=l_{1}}+\left.\left[\frac{\partial}{\partial s_{1}}\left(D_{\zeta_{1}} v_{1}^{\prime} v_{1}^{\prime \prime}\right)\right]\right|_{s_{1}=l_{1}}+} \\
+\left.\left[D_{\eta_{1}} w_{1}^{\prime \prime 2}\right]\right|_{s_{1}=l_{1}}+\left.\left[D_{\zeta_{1}} v_{1}^{\prime \prime 2}\right]\right|_{s_{1}=l_{1}}-\left.\left[\lambda_{1}\left(1+u_{1}^{\prime}\right)\right]\right|_{s_{1}=l_{1}}-\int_{0}^{l_{2}}\left[m_{2}\left(-\dot{v}_{1 c}^{\prime} \dot{v}_{1 c}-v_{1 c}^{\prime} \ddot{v}_{1 c}\right)+\right. \\
\left.+m_{2}\left(-\dot{w}_{1 c}^{\prime} \dot{V}_{2}-w_{1 c}^{\prime} \ddot{V}_{2}\right)+m_{2} \dot{v}_{1 c}^{\prime} \dot{v}_{1 c}+m_{2} \dot{V}_{2} \dot{w}_{1 c}^{\prime}+\tilde{q}_{z, 2}-c_{22} \dot{W}_{2}-c_{22} s_{2} \dot{v}_{1 C}^{\prime}+c_{22} \dot{u}_{1 C}\right] d s_{2}+ \\
+\left.\left[I_{\zeta_{2}} \delta_{2}\left(\dot{\phi}_{2} \dot{V}_{2}^{\prime}+\phi_{2} \ddot{V}_{2}^{\prime}+\dot{w}_{1 c}^{\prime} \dot{V}_{2}^{\prime}+w_{1 c}^{\prime} \ddot{V}_{2}^{\prime}\right)\right]\right|_{S_{2}=0}-\left[I _ { \eta _ { 2 } } \delta _ { 3 } \left(-\ddot{W}_{2}^{\prime}+\dot{\phi}_{2} \dot{V}_{2}^{\prime}+\phi_{2} \ddot{V}_{2}^{\prime}+\dot{w}_{1 c}^{\prime} \dot{V}_{2}^{\prime}+\right.\right. \\
\left.\left.+w_{1 c}^{\prime} \ddot{V}_{2}^{\prime}-\dot{\phi}_{2} \dot{\varphi}_{1 c}-\phi_{2} \ddot{\varphi}_{1 c}-\dot{w}_{1 c}^{\prime} \dot{\varphi}_{1 c}-w_{1 c}^{\prime} \ddot{\varphi}_{1 c}+\ddot{w}_{1 c}^{\prime} \varphi_{1 c}+\dot{w}_{1 c}^{\prime} \dot{\varphi}_{1 c}\right)\right]\left.\right|_{S_{2}=0}- \\
-\left.\left[I_{\xi_{2}}\left(\dot{V}_{2}^{\prime} \dot{\phi}_{2}-\dot{\varphi}_{1 c} \dot{\phi}_{2}\right)\right]\right|_{S_{2}=0}+\left.\left[\lambda_{2}\left(W_{2}^{\prime}+v_{1 C}^{\prime}\right)\right]\right|_{s_{2}=0}+\left.\left\{\frac{\partial}{\partial s_{2}}\left[D_{\eta_{2}}\left(-W_{2}^{\prime \prime}+\phi_{2} V_{2}^{\prime \prime}+w_{1 C}^{\prime} V_{2}^{\prime \prime}\right)\right]\right\}\right|_{S_{2}=0}- \\
-\left.\left\{\frac{\partial}{\partial s_{2}}\left[D_{\zeta_{2}}\left(V_{2}^{\prime \prime} \phi_{2}+V_{2}^{\prime \prime} w_{1 C}^{\prime}\right)\right]\right\}\right|_{S_{2}=0}+\left.\left[D_{\xi_{2}} \phi_{2}^{\prime} V_{2}^{\prime \prime}\right]\right|_{S_{2}=0}+M_{z, 2}(0, t)=0 .
\end{gathered}
$$

b) In-plane bending

-The first boundary condition arises by summing the terms from equations (B.10 a,b), which correspond to $\delta v_{1 C}$, and then by setting them equal to zero,

$$
\begin{gathered}
\left\{-I_{\xi_{1}}\left(\dot{w}_{1}^{\prime} \dot{\varphi}_{1}+w_{1}^{\prime} \ddot{\varphi}_{1}\right)+I_{\eta_{1}} \delta_{1}\left(\dot{\varphi}_{1} \dot{w}_{1}^{\prime}+\varphi_{1} \ddot{w}_{1}^{\prime}\right)-I_{\zeta_{1}} \delta_{3}\left(\ddot{v}_{1}^{\prime}+\ddot{w}_{1}^{\prime} \varphi_{1}+\dot{\varphi}_{1} \dot{w}_{1}^{\prime}\right)-\right. \\
\left.-\lambda_{1} v_{1}^{\prime}+\frac{\partial}{\partial s_{1}}\left[D_{\xi_{1}} w_{1}^{\prime} \varphi_{1}^{\prime}\right]-\frac{\partial}{\partial s_{1}}\left[D_{\eta_{1}} \varphi_{1} w_{1}^{\prime \prime}\right]+\frac{\partial}{\partial s_{1}}\left[D_{\zeta_{1}}\left(v_{1}^{\prime \prime}+\varphi_{1} w_{1}^{\prime \prime}\right)\right]+M_{y, 1}\right\}\left.\right|_{s_{1}=l_{1}}-
\end{gathered}
$$




$$
\begin{gathered}
-\int_{0}^{l_{2}}\left\{m _ { 2 } \left[\ddot{v}_{1 c}+\ddot{u}_{2}+\ddot{v}_{1 c}^{\prime} W_{2}+\dot{v}_{1 c}^{\prime} \dot{W}_{2}+s_{2} \ddot{v}_{1 c}^{\prime} v_{1 c}^{\prime}+s_{2} \dot{v}_{1 c}^{\prime} \dot{v}_{1 c}^{\prime}-\ddot{\varphi}_{1 c} V_{2}-\dot{\varphi}_{1 c} \dot{V}_{2}+s_{2} \dot{\varphi}_{1 c} \dot{\varphi}_{1 c}+\right.\right. \\
+s_{2} \varphi_{1 c} \ddot{\varphi}_{1 c}+\dot{\varphi}_{1 c} \dot{W}_{1 c}+\varphi_{1 c} \ddot{W}_{1 c}+\ddot{\varphi}_{1 c} w_{1 c}+\dot{\varphi}_{1 c} \dot{W}_{1 c}- \\
\left.\left.-\ddot{V}_{2} \varphi_{1 c}-\dot{V}_{2} \dot{\varphi}_{1 c}+\dot{v}_{1 c}^{\prime} \dot{W}_{2}+v_{1 c}^{\prime} \ddot{W}_{2}\right]\right\} d s_{2}=0 .
\end{gathered}
$$

-The second boundary condition, arises using equations (B.10c-e) in a similar way but corresponding to $\delta v_{1 C}^{\prime}$,

$$
\begin{gathered}
-\left.\left[D_{\xi_{1}} w_{1}^{\prime} \varphi_{1}^{\prime}\right]\right|_{s_{1}=l_{1}}+\left.\left[D_{\eta_{1}} \varphi_{1} w_{1}^{\prime \prime}\right]\right|_{s_{1}=l_{1}}-\left.\left[D_{\zeta_{1}}\left(v_{1}^{\prime \prime}+\varphi_{1} w_{1}^{\prime \prime}\right)\right]\right|_{s_{1}=l_{1}}-M_{z, p}- \\
-\int_{0}^{l_{2}}\left\{m_{2}\left(\dot{W}_{2} \dot{v}_{1 c}+W_{2} \ddot{v}_{1 c}+s_{2} \dot{v}_{1 c}^{\prime} \dot{v}_{1 c}+s_{2} v_{1 c}^{\prime} \ddot{v}_{1 c}\right)+m_{2}\left(\ddot{V}_{2} s_{2} w_{1 c}^{\prime}+\dot{V}_{2} s_{2} \dot{w}_{1 c}^{\prime}\right)+\right. \\
+\delta_{1} I_{\xi_{2}}\left(\delta_{1} \dot{\varphi}_{1 c} \dot{\phi}_{2}+\delta_{1} \varphi_{1 c} \ddot{\phi}_{2}\right)+\delta_{2} I_{\zeta_{2}}\left(\ddot{V}_{2}^{\prime} \phi_{2}+\dot{V}_{2}^{\prime} \dot{\phi}_{2}+2 \dot{w}_{1 c}^{\prime} \dot{V}_{2}^{\prime}+2 w_{1 c}^{\prime} \ddot{V}_{2}^{\prime}\right)- \\
-m_{2} s_{2} \dot{v}_{1 c}^{\prime} \dot{v}_{1 c}-m_{2} s_{2} \dot{w}_{1 c}^{\prime} \dot{V}_{2}-m_{2} \dot{v}_{1 c} \dot{W}_{2}-I_{\xi_{2}}\left(\dot{\phi}_{2} \dot{V}_{2}^{\prime}-\dot{\varphi}_{1 c} \dot{\phi}_{2}\right)+\lambda_{2}\left(W_{2}^{\prime}+v_{1 C}^{\prime}\right)+ \\
\left.+D_{\xi_{2}} V_{2}^{\prime \prime} \phi_{2}^{\prime}-s_{2} q_{z, 2}+M_{z, 2}\right\} d s_{2}+\left.\left[D_{\eta_{2}}\left(-W_{2}^{\prime \prime}+\phi_{2} V_{2}^{\prime \prime}+w_{1 c}^{\prime} V_{2}^{\prime \prime}\right)-D_{\zeta_{2}}\left(V_{2}^{\prime \prime} \phi_{2}+V_{2}^{\prime \prime} w_{1 C}^{\prime}\right)\right]\right|_{s_{2}=0}=0 .
\end{gathered}
$$

c) Out-of-plane bending, -the first boundary condition arises in a similar way as before but uses equations (B.11a-c) for $\delta w_{1 C}$ considering also equation (23g),

$$
\begin{gathered}
{\left.\left[I_{\eta_{1}} \delta_{1}\left(-\ddot{w}_{1}^{\prime}+\dot{\varphi}_{1} \dot{v}_{1}^{\prime}+\varphi_{1} \ddot{v}_{1}^{\prime}\right)\right]\right|_{s_{1}=l_{1}}-\left.\left[I_{\zeta_{1}} \delta_{3}\left(\dot{\varphi}_{1} \dot{v}_{1}^{\prime}+\varphi_{1} \ddot{v}_{1}^{\prime}\right)\right]\right|_{s_{1}=l_{1}}+\left.\left[I_{\xi_{1}} \dot{v}_{1}^{\prime} \dot{\varphi}_{1}\right]\right|_{s_{1}=l_{1}}-\left.\left[\lambda_{1} w_{1}^{\prime}\right]\right|_{s_{1}=l_{1}}-} \\
-\left.\left\{\frac{\partial}{\partial s_{1}}\left[D_{\eta_{1}}\left(-w_{1}^{\prime \prime}+\varphi_{1} v_{1}^{\prime \prime}\right)\right]\right\}\right|_{s_{1}=l_{1}}+\left.\left\{\frac{\partial}{\partial s_{1}}\left(D_{\zeta_{1}} \varphi_{1} v_{1}^{\prime \prime}\right)\right\}\right|_{s_{1}=l_{1}}-\left.\left[D_{\xi_{1}} v_{1}^{\prime \prime} \varphi_{1}^{\prime}\right]\right|_{s_{1}=l_{1}}-M_{z, 1}\left(l_{1}, t\right)- \\
\quad-\int_{0}^{l_{2}}\left\{m_{2}\left(\dot{\varphi}_{1 c} \dot{v}_{1 c}+\varphi_{1 c} \ddot{v}_{1 c}\right)+m_{2}\left(\dot{w}_{1 c}^{\prime} \dot{W}_{2}+w_{1 c}^{\prime} \ddot{W}_{2}\right)-\right. \\
\left.+m_{2} \dot{\varphi}_{1 c} \dot{v}_{1 c}-m_{2} \dot{W}_{1 c}^{\prime} \dot{W}_{2}+\tilde{q}_{y, 2}-c_{23} \dot{V}_{2}+c_{23} s_{2} \dot{\varphi}_{1 C}+c_{23} \dot{W}_{1 C}\right\} d s_{2}+ \\
+\left.\left[I_{\xi_{2}}\left(\dot{W}_{2}^{\prime} \dot{\phi}_{2}+W_{2}^{\prime} \ddot{\phi}_{2}+\dot{v}_{1 c}^{\prime} \dot{\phi}_{2}+v_{1 c}^{\prime} \ddot{\phi}_{2}\right)\right]\right|_{S_{2}=0} ^{\prime}+\left.\left[I_{\zeta_{2}} \delta_{2}\left(\ddot{V}_{2}^{\prime}+\ddot{W}_{2}^{\prime} \dot{\phi}_{2}+\dot{\phi}_{2}+\dot{W}_{2}^{\prime}+\ddot{v}_{1 c}^{\prime} \phi_{2}+\dot{v}_{1 c}^{\prime}+2 \dot{v}_{1 c}^{\prime} \dot{w}_{1 c}^{\prime}\right)\right]\right|_{S_{2}=0}-\left.\left[I_{\eta_{2}} \delta_{3}\left(\dot{\phi}_{2} \dot{W}_{2}^{\prime}+\phi_{2} \ddot{W}_{2}^{\prime}+\dot{w}_{1 c}^{\prime} \dot{W}_{2}^{\prime}+w_{1 c}^{\prime} \ddot{W}_{2}^{\prime}\right)\right]\right|_{S_{2}=0}+ \\
+\left.\left[\lambda_{2}\left(V_{2}^{\prime}-\varphi_{1 C}\right)\right]\right|_{s_{2}=0}-\left.\left\{\frac{\partial}{\partial s_{2}}\left[D_{\xi_{2}}\left(W_{2}^{\prime} \phi_{2}^{\prime}+v_{1 C}^{\prime} \phi_{2}^{\prime}\right)\right]\right\}\right|_{S_{2}=0}+\left.\left\{\frac{\partial}{\partial s_{2}}\left[D_{\eta_{2}}\left(\phi_{2} W_{2}^{\prime \prime}+w_{1 C}^{\prime} W_{2}^{\prime \prime}\right)\right]\right\}\right|_{S_{2}=0}- \\
-\left.\left\{\frac{\partial}{\partial s_{2}}\left[D_{\zeta_{2}}\left(V_{2}^{\prime \prime}+\phi_{2} W_{2}^{\prime \prime}+w_{1 C}^{\prime} W_{2}^{\prime \prime}\right)\right]\right\}\right|_{s_{2}=0}-M_{y, 2}(0, t)-q_{y, p}=0 .
\end{gathered}
$$

-The second boundary condition emerges by using equations (B.11d-f) which correspond to $\delta w_{1 C}^{\prime}$,

$$
\left.\left[D_{\eta_{1}}\left(-w_{1}^{\prime \prime}+\varphi_{1} v_{1}^{\prime \prime}\right)\right]\right|_{S_{1}=l_{1}}-\left.\left[D_{\zeta_{1}}\left(\varphi_{1} v_{1}^{\prime \prime}\right)\right]\right|_{S_{1}=l_{1}}+M_{x, p}-
$$




$$
\begin{gathered}
-\int_{0}^{l_{2}}\left\{m_{2}\left(\ddot{V}_{2} W_{2}+\dot{V}_{2} \dot{W}_{2}+s_{2} \dot{v}_{1 c}^{\prime} \dot{V}_{2}+s_{2} v_{1 c}^{\prime} \ddot{V}_{2}\right)+m_{2}\left(-\ddot{W}_{2} V_{2}-\dot{W}_{2} \dot{V}_{2}+\ddot{W}_{2} w_{1 c}+\dot{W}_{2} \dot{W}_{1 c}\right)-\right. \\
-I_{\eta_{2}} \delta_{3}\left(\ddot{W}_{2}^{\prime} \varphi_{1 c}+\dot{W}_{2}^{\prime} \dot{\varphi}_{1 c}\right)-m_{2} s_{2} \dot{v}_{1 c}^{\prime} \dot{V}_{2}-m_{2} \dot{W}_{2} \dot{W}_{1 c}-I_{\zeta_{2}} \delta_{2}\left(\dot{V}_{2}^{\prime} \dot{W}_{2}^{\prime}+2 \dot{v}_{1 c}^{\prime} \dot{V}_{2}^{\prime}\right)- \\
-I_{\eta_{2}} \delta_{3}\left(-\dot{V}_{2}^{\prime} \dot{W}_{2}^{\prime}+\dot{\varphi}_{1 c} \dot{W}_{2}^{\prime}\right)-D_{\eta_{2}} W_{2}^{\prime \prime} V_{2}^{\prime \prime}+ \\
\left.+D_{\zeta_{2}} W_{2}^{\prime \prime} V_{2}^{\prime \prime}-M_{x, 2}\right\} d s_{2}-\left.\left[D_{\xi_{2}}\left(\phi_{2}^{\prime}+V_{2}^{\prime \prime} W_{2}^{\prime}+v_{1 c}^{\prime} V_{2}^{\prime \prime}\right)\right]\right|_{S_{2}=0}=0 .
\end{gathered}
$$

d) Torsional motion,

-Using equations (B.12a-c) which correspond to $\delta \varphi_{1 C}$,

$$
\begin{gathered}
-\left.\left[D_{\xi_{1}}\left(\varphi_{1}^{\prime}+v_{1}^{\prime \prime} w_{1}^{\prime}\right)\right]\right|_{s_{1}=l_{1}}-l_{2} q_{y, p}-M_{y, p}-\int_{0}^{l_{2}}\left\{m _ { 2 } \left(-\dot{V}_{2} \dot{v}_{1 c}-V_{2} \ddot{v}_{1 c}+s_{2} \dot{\varphi}_{1 c} \dot{v}_{1 c}+\right.\right. \\
\left.+s_{2} \varphi_{1 c} \ddot{v}_{1 c}+\dot{w}_{1 c} \dot{v}_{1 c}+w_{1 c} \ddot{v}_{1 c}\right)-I_{\xi_{2}}\left(\ddot{\phi}_{2} W_{2}^{\prime}+\dot{\phi}_{2} \dot{W}_{2}^{\prime}+\ddot{\phi}_{2} v_{1 C}^{\prime}+\dot{\phi}_{2} \dot{v}_{1 C}^{\prime}\right)- \\
+I_{\eta_{2}} \delta_{3}\left(\ddot{W}_{2}^{\prime} \phi_{2}+\dot{W}_{2}^{\prime} \dot{\phi}_{2}+\ddot{W}_{2}^{\prime} w_{1 c}^{\prime}+\dot{W}_{2}^{\prime} \dot{w}_{1 c}^{\prime}\right)- \\
-m_{2}\left(s_{2} \dot{\varphi}_{1 c} \dot{v}_{1 c}+\dot{v}_{1 c} \dot{w}_{1 c}\right)+m_{2} \dot{v}_{1 c} \dot{V}_{2}-I_{\xi_{2}} \delta_{1} \dot{\phi}_{2} \dot{v}_{1 c}^{\prime}+I_{\eta_{2}} \delta_{3} \dot{W}_{2}^{\prime} \dot{w}_{1 c}^{\prime}- \\
\left.-\lambda_{2}\left(V_{2}^{\prime}-\varphi_{1 c}\right)+s_{2} q_{y, 2}+M_{y, 2}\right\} d s_{2}+\left.\left[D_{\xi_{2}}\left(W_{2}^{\prime} \phi_{2}^{\prime}+v_{1 C}^{\prime} \phi_{2}^{\prime}\right)\right]\right|_{s_{2}=0}- \\
-\left.\left[D_{\eta_{2}}\left(\phi_{2} W_{2}^{\prime \prime}+w_{1 C}^{\prime} W_{2}^{\prime \prime}\right)\right]\right|_{s_{2}=0}+\left.\left[D_{\zeta_{2}}\left(V_{2}^{\prime \prime}+\phi_{2} W_{2}^{\prime \prime}+w_{1 c}^{\prime} W_{2}^{\prime \prime}\right)\right]\right|_{s_{2}=0}=0 .
\end{gathered}
$$

-Secondary beam,

a) For axial motion using equation (B.13a), then the boundary condition is,

$$
\begin{gathered}
{\left.\left[I_{\eta_{2}} \delta_{3}\left(-W_{2}^{\prime} \ddot{W}_{2}^{\prime}+v_{1 c} \ddot{W}_{2}^{\prime}\right)\right]\right|_{S_{2}=l_{2}}+\left.\left[I_{\zeta_{2}} \delta_{2}\left(-\ddot{V}_{2}^{\prime} V_{2}^{\prime}+\varphi_{1 c} \ddot{V}_{2}^{\prime}\right)\right]\right|_{S_{2}=l_{2}}+} \\
+\left.\left\{\frac{\partial}{\partial s_{2}}\left[D_{\eta_{2}}\left(W_{2}^{\prime} W_{2}^{\prime \prime}+v_{1 c}^{\prime} W_{2}^{\prime \prime}\right)\right]\right\}\right|_{S_{2}=l_{2}}-\left.\left\{\frac{\partial}{\partial s_{2}}\left[D_{\zeta_{2}}\left(-V_{2}^{\prime} V_{2}^{\prime \prime}+\varphi_{1 c} V_{2}^{\prime \prime}\right)\right]\right\}\right|_{S_{2}=l_{2}}- \\
-\left.\left[D_{\eta_{2}} W_{2}^{\prime \prime 2}\right]\right|_{S_{2}=l_{2}}-\left.\left[D_{\zeta_{2}} V_{2}^{\prime \prime 2}\right]\right|_{S_{2}=l_{2}}-\left.\left[\lambda_{2}\left(1+u_{2}^{\prime}\right)\right]\right|_{S_{2}=l_{2}}=0 .
\end{gathered}
$$

b) For in-plane bending,

-The first boundary condition is from equation (B.14a),

$$
\begin{gathered}
-\left.\left[I_{\zeta_{2}} \delta_{2}\left(\dot{\phi}_{2} \dot{V}_{2}^{\prime}+\phi_{2} \ddot{V}_{2}^{\prime}+\dot{w}_{1 c}^{\prime} \dot{V}_{2}^{\prime}+w_{1 c}^{\prime} \ddot{V}_{2}^{\prime}\right)\right]\right|_{s_{2}=l_{2}}+\left[I _ { \eta _ { 2 } } \delta _ { 3 } \left(-\ddot{W}_{2}^{\prime}+\dot{\phi}_{2} \dot{V}_{2}^{\prime}+\phi_{2} \ddot{V}_{2}^{\prime}+\dot{w}_{1 c}^{\prime} \dot{V}_{2}^{\prime}+\right.\right. \\
\left.\left.+w_{1 c}^{\prime} \ddot{V}_{2}^{\prime}-\dot{\phi}_{2} \dot{\varphi}_{1 c}-\phi_{2} \ddot{\varphi}_{1 c}-\dot{w}_{1 c}^{\prime} \dot{\varphi}_{1 c}-w_{1 c}^{\prime} \ddot{\varphi}_{1 c}+\ddot{w}_{1 c}^{\prime} \varphi_{1 c}+\dot{w}_{1 c}^{\prime} \dot{\varphi}_{1 c}\right)\right]\left.\right|_{s_{2}=l_{2}}+ \\
+\left.\left[I_{\xi_{2}}\left(\dot{V}_{2}^{\prime} \dot{\phi}_{2}-\dot{\varphi}_{1 c} \dot{\phi}_{2}\right)\right]\right|_{S_{2}=l_{2}}-\left.\left[\lambda_{2}\left(W_{2}^{\prime}+v_{1 c}^{\prime}\right)\right]\right|_{s_{2}=l_{2}}-\left.\left\{\frac{\partial}{\partial s_{2}}\left[D_{\eta_{2}}\left(-W_{2}^{\prime \prime}+\phi_{2} V_{2}^{\prime \prime}+w_{1 c}^{\prime} V_{2}^{\prime \prime}\right)\right]\right\}\right|_{S_{2}=l_{2}}+ \\
+\left.\left\{\frac{\partial}{\partial s_{2}}\left[D_{\zeta_{2}}\left(V_{2}^{\prime \prime} \phi_{2}+V_{2}^{\prime \prime} w_{1 C}^{\prime}\right)\right]\right\}\right|_{S_{2}=l_{2}}-\left.\left[D_{\xi_{2}} \phi_{2}^{\prime} V_{2}^{\prime \prime}\right]\right|_{s_{2}=l_{2}}-M_{z, 2}\left(l_{2}, t\right)=0
\end{gathered}
$$

-The second boundary condition comes from equation (B.14b),

$$
\left.\left[D_{\eta_{2}}\left(-W_{2}^{\prime \prime}+\phi_{2} V_{2}^{\prime \prime}+w_{1 C}^{\prime} V_{2}^{\prime \prime}\right)\right]\right|_{S_{2}=l_{2}}-\left.\left[D_{\zeta_{2}}\left(V_{2}^{\prime \prime} \phi_{2}+V_{2}^{\prime \prime} w_{1 C}^{\prime}\right)\right]\right|_{S_{2}=l_{2}}-M_{z, p}=0
$$


c) For out-of-plane bending,

-the first boundary condition is determined by using equation (B.15a),

$$
\begin{aligned}
-\left.\left[I_{\xi_{2}}\left(\dot{W}_{2}^{\prime} \dot{\phi}_{2}+W_{2}^{\prime} \ddot{\phi}_{2}+\dot{v}_{1 c}^{\prime} \dot{\phi}_{2}+v_{1 c}^{\prime} \ddot{\phi}_{2}\right)\right]\right|_{S_{2}=l_{2}}-\left[I _ { \zeta _ { 2 } } \delta _ { 2 } \left(\ddot{V}_{2}^{\prime}+\ddot{W}_{2}^{\prime} \phi_{2}+\dot{\phi}_{2} \dot{W}_{2}^{\prime}+\ddot{v}_{1 c}^{\prime} \phi_{2}+\dot{v}_{1 c}^{\prime} \dot{\phi}_{2}+\right.\right. \\
\left.\left.+\ddot{W}_{2}^{\prime} w_{1 c}^{\prime}+\dot{W}_{2}^{\prime} \dot{w}_{1 c}^{\prime}+2 \ddot{v}_{1 c}^{\prime} w_{1 c}^{\prime}+2 \dot{v}_{1 c}^{\prime} \dot{W}_{1 c}^{\prime}\right)\right]\left.\right|_{S_{2}=l_{2}}- \\
-\left.\left[I_{\eta_{2}} \delta_{3}\left(\dot{\phi}_{2} \dot{W}_{2}^{\prime}+\phi_{2} \ddot{W}_{2}^{\prime}-\dot{w}_{1 c}^{\prime} \dot{W}_{2}^{\prime}-w_{1 c}^{\prime} \ddot{W}_{2}^{\prime}\right)\right]\right|_{s_{2}=l_{2}}-\left.\left[\lambda_{2}\left(V_{2}^{\prime}-\varphi_{1 C}\right)\right]\right|_{s_{2}=l_{2}}+ \\
+\left.\left\{\frac{\partial}{\partial s_{2}}\left[D_{\xi_{2}}\left(W_{2}^{\prime} \phi_{2}^{\prime}+v_{1 c}^{\prime} \phi_{2}^{\prime}\right)\right]\right\}\right|_{S_{2}=l_{2}}-\left.\left\{\frac{\partial}{\partial s_{2}}\left[D_{\eta_{2}}\left(\phi_{2} W_{2}^{\prime \prime}+w_{1 C}^{\prime} W_{2}^{\prime \prime}\right)\right]\right\}\right|_{S_{2}=l_{2}}+ \\
+\left.\left\{\frac{\partial}{\partial s_{2}}\left[D_{\zeta_{2}}\left(V_{2}^{\prime \prime}+\phi_{2} W_{2}^{\prime \prime}+w_{1 C}^{\prime} W_{2}^{\prime \prime}\right)\right]\right\}\right|_{S_{2}=l_{2}}+M_{y, 2}\left(l_{2}, t\right)+q_{y, p}=0 .
\end{aligned}
$$

-The second boundary condition is obtained using equation (B.15b),

$$
\begin{aligned}
- & {\left.\left[D_{\xi_{2}}\left(W_{2}^{\prime} \phi_{2}^{\prime}+v_{1 C}^{\prime} \phi_{2}^{\prime}\right)\right]\right|_{S_{2}=l_{2}}+\left.\left[D_{\eta_{2}}\left(\phi_{2} W_{2}^{\prime \prime}+w_{1 C}^{\prime} W_{2}^{\prime \prime}\right)\right]\right|_{S_{2}=l_{2}}-} \\
- & {\left.\left[D_{\zeta_{2}}\left(V_{2}^{\prime \prime}+\phi_{2} W_{2}^{\prime \prime}+w_{1 C}^{\prime} W_{2}^{\prime \prime}\right)\right]\right|_{S_{2}=l_{2}}+M_{y, p}=0 . }
\end{aligned}
$$

d) Torsional motion,

The boundary condition is determined using equation (B.16a),

$$
\left.\left[D_{\xi_{2}}\left(\phi_{2}^{\prime}+V_{2}^{\prime \prime} W_{2}^{\prime}+v_{1 C}^{\prime} V_{2}^{\prime \prime}\right)\right]\right|_{S_{2}=l_{2}}-M_{x, p}=0
$$

Also the inextensionality conditions are given by,

$$
\left(1+u_{1}^{\prime}\right)^{2}+\left(w_{1}^{\prime}\right)^{2}+\left(v_{1}^{\prime}\right)^{2}=1,\left(1+u_{2}^{\prime}\right)^{2}+\left(W_{2}^{\prime}+v_{1 C}^{\prime}\right)^{2}+\left(V_{2}^{\prime}-\varphi_{1 C}\right)^{2}=1 .
$$

Expanding them by means of a Taylor series and keeping up to second order terms leads to,

$$
u_{1}^{\prime}=-\frac{1}{2}\left(v_{1}^{\prime 2}+w_{1}^{\prime 2}\right)
$$

and

$$
u_{2}^{\prime}=-\frac{1}{2}\left(\varphi_{1 C}^{2}+v_{1 c}^{\prime 2}-2 \varphi_{1 C} V_{2}^{\prime}+{V_{2}^{\prime 2}}^{2}+2 v_{1 C}^{\prime} W_{2}^{\prime}+{W_{2}^{\prime}}^{2}\right) .
$$

Therefore, by means of spatial integration of equations $(65 \mathrm{a}, \mathrm{b})$ and then considering the strong boundary conditions (eq. 36,40 ) the axial displacements are given by,

$$
u_{1}=-\frac{1}{2} \int_{0}^{s_{1}}\left(v_{1}^{\prime^{2}}+{w_{1}^{\prime 2}}^{2}\right) d s_{1}
$$

and

$$
u_{2}=-\frac{1}{2} \int_{0}^{s_{2}}\left(\varphi_{1 C}^{2}+{v_{1 c}^{\prime}}^{2}-2 \varphi_{1 C} V_{2}^{\prime}+{V_{2}^{\prime}}^{2}+2 v_{1 C}^{\prime} W_{2}^{\prime}+{W_{2}^{\prime}}^{2}\right) d s_{2}
$$

It arises from equations $(66 a, b)$ that the axial displacements are in the form of second order nonlinearities. 
The initial conditions can be arbritrarily chosen for the full set of equations (24-31) considering the fact that model represents oscillations of medium amplitude.

\section{Orthogonality of the associated linear modes}

In this section, it is proven that the underlying linear system modes are orthogonal to each other by showing that the linear operators for the two sets of coupled linear equations are self-adjoint in case of considering constant properties of the beam in longitudinal direction. The orthogonality property is rather essential for deriving a reduced order model based in the projection of the nonlinear system in the infinite basis of the linear modes of the underlying linear system.

-In-plane motion.

It was shown in Georgiades et al. (2013b) that the rotary inertia terms can be neglected, therefore the linear equations are given by,

$$
m_{1} \ddot{v}_{1}+D_{\zeta_{1}} v_{1}^{I V}=0, \quad m_{2} \ddot{W}_{2}+D_{\eta_{2}} W_{2}^{I V}=0,
$$

with boundary conditions,

$$
\begin{gathered}
v_{1}(0, t)=0, \quad v_{1}^{\prime}(0, t)=0, \quad D_{\zeta_{1}} v_{1}^{\prime \prime \prime}\left(l_{1}, t\right)=l_{2} m_{2} \ddot{v}_{1}\left(l_{1}, t\right), \quad D_{\zeta_{1}} v_{1}^{\prime \prime}\left(l_{1}, t\right)=-D_{\eta_{2}} W_{2}^{\prime \prime}(0, t), \\
W_{2}(0, t)=0, \quad W_{2}^{\prime}(0, t)=-v_{1}^{\prime}\left(l_{1}, t\right), \quad W_{2}^{\prime \prime}\left(l_{2}, t\right)=0, \quad W_{2}^{\prime \prime \prime}\left(l_{2}, t\right)=0 . \quad(68 \mathrm{a}-\mathrm{h}),
\end{gathered}
$$

Balachandran and Nayfeh in (1990) examined a similar system describing the in-plane motion of an LShaped beam structure including also tip masses and it was shown that the system is self adjoint. By setting to zero the tip masses the system described in the work of Balachandran and Nayfeh (1990) is found to be the same as the system described in equations $(66,67)$. Therefore equations $(66,67)$ which describe the in-plane motion form a self-adjoint linear system.

-Out-of-plane motion,

The linear equations are given by,

$$
\begin{aligned}
m_{1} \ddot{w}_{1}-I_{\eta_{1}} \ddot{w}_{1}^{\prime \prime}+D_{\eta_{1}} w_{1}^{I V}=0, & m_{2} \ddot{V}_{2}-I_{\zeta_{2}} \ddot{V}_{2}^{\prime \prime}+D_{\zeta_{2}} V_{2}^{I V}=0, \\
I_{\xi_{1}} \ddot{\varphi}_{1}-D_{\xi_{1}} \varphi_{1}^{\prime \prime}=0, & I_{\xi_{2}} \ddot{\phi}_{2}-D_{\xi_{2}} \phi_{2}^{\prime \prime}=0,
\end{aligned}
$$

with boundary conditions,

$$
\begin{gathered}
w_{1}(0, t)=0, \quad w_{1}^{\prime}(0, t)=0, \\
-I_{\eta_{1}} \ddot{w}_{1}^{\prime}\left(l_{1}, t\right)+D_{\eta_{1}} w_{1}^{\prime \prime \prime}\left(l_{1}, t\right)+I_{\zeta_{2}} \ddot{\varphi}_{1}\left(l_{1}, t\right)-D_{\zeta_{2}} V_{2}^{\prime \prime \prime}(0, t)=0, \\
D_{\eta_{1}} w_{1}^{\prime \prime}\left(l_{1}, t\right)+D_{\xi_{2}} \phi_{2}^{\prime}(0, t)=0, \\
\varphi_{1}(0, t)=0, \\
D_{\xi_{1}} \varphi_{1}^{\prime}\left(l_{1}, t\right)+I_{\zeta_{2}} \ddot{w}_{1}\left(l_{1}, t\right)-I_{\zeta_{2}} \ddot{V}_{2}\left(l_{2}, t\right)-D_{\zeta_{2}} V_{2}^{\prime \prime}(0, t)-\frac{I_{\zeta_{2}}^{2}}{m_{2}} \ddot{V}_{2}^{\prime \prime}(0, t)- \\
-\frac{I_{\zeta_{2}} D_{\zeta_{2}}}{m_{2}}\left[V_{2}^{I V}\left(l_{2}, t\right)-V_{2}^{I V}(0, t)\right]=0,
\end{gathered}
$$




$$
\begin{array}{cl}
V_{2}(0, t)=w_{1}\left(l_{1}, t\right), & V_{2}^{\prime}(0, t)=\varphi_{1}\left(l_{1}, t\right), \\
-I_{\zeta_{2}} \ddot{V}_{2}^{\prime}\left(l_{2}, t\right)+D_{\zeta_{2}} V_{2}^{\prime \prime \prime}\left(l_{2}, t\right)=0, & V_{2}^{\prime \prime}\left(l_{2}, t\right)=0, \\
\phi_{2}(0, t)=-w_{1}^{\prime}\left(l_{1}, t\right), & \phi_{2}^{\prime}\left(l_{2}, t\right)=0 .
\end{array}
$$

Using separation of variables in space and time again, as in (Bolotin 1964), then the equations in space and the associated boundary conditions take the form,

$$
\begin{gathered}
D_{\eta_{1}} Y_{w_{1}}^{I V}+I_{\eta_{1}} \omega_{\text {out }}^{2} Y_{w_{1}}^{\prime \prime}-\omega_{\text {out }}^{2} m_{1} Y_{w_{1}}=0, \quad D_{\zeta_{2}} Y_{V_{2}}^{I V}+\omega_{\text {out }}^{2} I_{\zeta_{2}} Y_{V_{2}}^{\prime \prime}-\omega_{\text {out }}^{2} m_{2} Y_{V_{2}}=0 \\
D_{\xi_{1}} Y_{\varphi_{1}}^{\prime \prime}+\omega_{\text {out }}^{2} I_{\xi_{1}} Y_{\varphi_{1}}=0,
\end{gathered}
$$

with the boundary conditions,

$$
\begin{aligned}
& Y_{w_{1}}(0)=0, \quad Y_{w_{1}}^{\prime}(0)=0, \\
& \omega_{\text {out }}^{2} I_{\eta_{1}} Y_{w_{1}}^{\prime}\left(l_{1}\right)+D_{\eta_{1}} Y_{w_{1}}^{\prime \prime \prime}\left(l_{1}\right)-I_{\zeta_{2}} \omega_{o u t}^{2} Y_{\varphi_{1}}\left(l_{1}\right)-D_{\zeta_{2}} Y_{V_{2}}^{\prime \prime \prime}(0)=0 \\
& D_{\eta_{1}} Y_{w_{1}}^{\prime \prime}\left(l_{1}\right)+D_{\xi_{2}} Y_{\Phi_{2}}^{\prime}(0)=0 \\
& Y_{\varphi_{1}}(0)=0, \\
& D_{\xi_{1}} Y_{\varphi_{1}}^{\prime}\left(l_{1}\right)-\omega_{\text {out }}^{2} I_{\zeta_{2}} Y_{w_{1}}\left(l_{1}\right)+I_{\zeta_{2}} \omega_{\text {out }}^{2} Y_{V_{2}}\left(l_{2}\right)-D_{\zeta_{2}} Y_{V_{2}}^{\prime \prime}(0)+\omega_{\text {out }}^{2} \frac{I_{\zeta_{2}}^{2}}{m_{2}} Y_{V_{2}}^{\prime \prime}(0)- \\
& -\frac{I_{\zeta_{2}} D_{\zeta_{2}}}{m_{2}}\left[Y_{V_{2}}^{I V}\left(l_{2}\right)-Y_{V_{2}}^{I V}(0)\right]=0, \\
& Y_{V_{2}}(0)=Y_{w_{1}}\left(l_{1}\right), \quad Y_{V_{2}}^{\prime}(0)=Y_{\varphi_{1}}\left(l_{1}\right), \\
& \omega_{\text {out }}^{2} I_{\zeta_{2}} Y_{V_{2}}^{\prime}\left(l_{2}\right)+D_{\zeta_{2}} Y_{V_{2}}^{\prime \prime \prime}\left(l_{2}\right)=0, \quad Y_{V_{2}}^{\prime \prime}\left(l_{2}\right)=0 \text {, } \\
& Y_{\Phi_{2}}(0)=-Y_{w_{1}}^{\prime}\left(l_{1}\right), \quad Y_{\Phi_{2}}^{\prime}\left(l_{2}\right)=0 .
\end{aligned}
$$

Following the same process as in Balachandran and Nayfeh (1990a), the linear operators $L_{1}, L_{2}, L_{3}$, $\mathrm{L}_{4}$, associated with out-of-plane motion, are given by,

$$
\begin{array}{ccc}
L_{1}=\left[-m_{1} \omega_{\text {out }}^{2}+I_{\eta_{1}} \omega_{\text {out }}^{2} \frac{d^{2}}{d s_{1}^{2}}+D_{\eta_{1}} \frac{d^{4}}{d s_{1}^{4}}\right], & L_{2}=\left[-\omega_{\text {out }}^{2} m_{2}+\omega_{\text {out }}^{2} I_{\zeta_{2}} \frac{d^{2}}{d s_{2}^{2}}+D_{\zeta_{2}} \frac{d^{4}}{d s_{2}^{4}}\right], \\
L_{3}=\left[\omega_{\text {out }}^{2} I_{\xi_{1}}+D_{\xi_{1}} \frac{d^{2}}{d s_{1}^{2}}\right], & \text { and }, & L_{4}=\left[\omega_{\text {out }}^{2} I_{\xi_{2}}+D_{\xi_{2}} \frac{d^{2}}{d s_{2}^{2}}\right] .
\end{array}
$$

By considering four adjoint solutions $q_{w_{1}}, q_{v_{2}}, q_{\varphi_{1}}$ and $q_{\Phi_{2}}$ it has to be shown that (Nayfeh 1981),

$$
\begin{aligned}
\left(q_{w_{1}}, L_{1} Y_{w_{1}}\right)+\left(q_{v_{2}}, L_{2} Y_{V_{2}}\right)+ & \left(q_{\varphi_{1}}, L_{3} Y_{\varphi_{1}}\right)+\left(q_{\Phi_{2}}, L_{4} Y_{\Phi_{2}}\right)=\left(Y_{w_{1}}, L_{1} q_{w_{1}}\right)+\left(Y_{V_{2}}, L_{2} q_{v_{2}}\right)+ \\
& +\left(Y_{\varphi_{1}}, L_{3} q_{\varphi_{1}}\right)+\left(Y_{\Phi_{2}}, L_{4} q_{\Phi_{2}}\right),
\end{aligned}
$$


where $($,$) is the inner product, and then by expanding equation (80) and using the definition of the$ inner product it is shown that,

$$
\begin{aligned}
& \int_{0}^{l_{1}} q_{w_{1}} L_{1} Y_{w_{1}} d s_{1}+\int_{0}^{l_{2}} q_{v_{2}} L_{2} Y_{V_{2}} d s_{2} \int_{0}^{l_{1}} q_{\varphi_{1}} L_{3} Y_{\varphi_{1}} d s_{1}+\int_{0}^{l_{2}} q_{\Phi_{2}} L_{4} Y_{\Phi_{2}} d s_{2}= \\
= & \int_{0}^{l_{1}} Y_{w_{1}} L_{1} q_{w_{1}} d s_{1}+\int_{0}^{l_{2}} Y_{V_{2}} L_{2} q_{v_{2}} d s_{2}+\int_{0}^{l_{1}} Y_{\varphi_{1}} L_{3} q_{\varphi_{1}} d s_{1}+\int_{0}^{l_{2}} Y_{\Phi_{2}} L_{4} q_{\Phi_{2}} d s_{2} .
\end{aligned}
$$

In the case of a self-adjoint system it is clear that the terms associated with the boundary conditions from the integration by parts must vanish. By using the same boundary conditions for both solutions then the following equation should equate to zero,

$$
\begin{aligned}
& {\left.\left[q_{w_{1}} I_{\eta_{1}} \omega_{\text {out }}^{2} Y_{w_{1}}^{\prime}\right]\right|_{s_{1}=0} ^{s_{1}=l_{1}}-\left.\left[q_{w_{1}}^{\prime} I_{\eta_{1}} \omega_{\text {out }}^{2} Y_{w_{1}}\right]\right|_{s_{1}=0} ^{s_{1}=l_{1}}+\left.\left[q_{v_{2}} \omega_{\text {out }}^{2} I_{\zeta_{2}} Y_{V_{2}}^{\prime}\right]\right|_{s_{2}=0} ^{s_{2}=l_{2}}-\left.\left[q_{v_{2}}^{\prime} \omega_{\text {out }}^{2} I_{\zeta_{2}} Y_{V_{2}}\right]\right|_{S_{2}=0} ^{s_{2}=l_{2}}+} \\
& +\left.\left[q_{w_{1}} D_{\eta_{1}} Y_{w_{1}}^{\prime \prime \prime}\right]\right|_{s_{1}=0} ^{s_{1}=l_{1}}-\left.\left[q_{w_{1}}^{\prime} D_{\eta_{1}} Y_{w_{1}}^{\prime \prime}\right]\right|_{S_{1}=0} ^{s_{1}=l_{1}}+\left.\left[q_{w_{1}}^{\prime \prime} D_{\eta_{1}} Y_{w_{1}}^{\prime}\right]\right|_{s_{1}=0} ^{s_{1}=l_{1}}-\left.\left[q_{w_{1}}^{\prime \prime \prime} D_{\eta_{1}} Y_{w_{1}}\right]\right|_{s_{1}=0} ^{s_{1}=l_{1}}+ \\
& +\left.\left[q_{v_{2}} D_{\zeta_{2}} Y_{V_{2}}^{\prime \prime \prime}\right]\right|_{S_{2}=0} ^{s_{2}=l_{2}}-\left.\left[q_{v_{2}}^{\prime} D_{\zeta_{2}} Y_{V_{2}}^{\prime \prime}\right]\right|_{S_{2}=0} ^{s_{2}=l_{2}}+\left.\left[q_{v_{2}}^{\prime \prime} D_{\zeta_{2}} Y_{V_{2}}^{\prime}\right]\right|_{S_{2}=0} ^{s_{2}=l_{2}}-\left.\left[q_{v_{2}}^{\prime \prime \prime} D_{\zeta_{2}} Y_{V_{2}}\right]\right|_{S_{2}=0} ^{s_{2}=l_{2}}- \\
& -\left.\left[q_{\varphi_{1}} D_{\xi_{1}} Y_{\varphi_{1}}^{\prime}\right]\right|_{S_{1}=0} ^{s_{1}=l_{1}}+\left.\left[q_{\varphi_{1}}^{\prime} D_{\xi_{1}} Y_{\varphi_{1}}\right]\right|_{S_{1}=0} ^{s_{1}=l_{1}}-\left.\left[q_{\Phi_{2}} D_{\xi_{2}} Y_{\Phi_{2}}^{\prime}\right]\right|_{S_{2}=0} ^{s_{2}=l_{2}}+\left.\left[q_{\Phi_{2}}^{\prime} D_{\xi_{2}} Y_{\Phi_{2}}\right]\right|_{S_{2}=0} ^{s_{2}=l_{2}}=0 .
\end{aligned}
$$

The boundary conditions are given by equations $(75)-(78)$ and the boundary conditions of the adjoint solution take the form,

$$
\begin{gathered}
q_{w_{1}}(0)=0, \quad q_{w_{1}}^{\prime}(0)=0, \\
\omega_{o u t}^{2} I_{\eta_{1}} q_{w_{1}}^{\prime}\left(l_{1}\right)+D_{\eta_{1}} q_{w_{1}}^{\prime \prime \prime}\left(l_{1}\right)-I_{\zeta_{2}} \omega_{o u t}^{2} q_{\varphi_{1}}\left(l_{1}\right)-D_{\zeta_{2}} q_{v_{2}}^{\prime \prime \prime}(0)=0, \\
D_{\eta_{1}} q_{w_{1}}^{\prime \prime}\left(l_{1}\right)+D_{\xi_{2}} q_{\Phi_{2}}^{\prime}(0)=0, \\
q_{\varphi_{1}}(0)=0, \\
D_{\xi_{1}} q_{\varphi_{1}}^{\prime}\left(l_{1}\right)-\omega_{\text {out }}^{2} I_{\zeta_{2}} q_{w_{1}}\left(l_{1}\right)+I_{\zeta_{2}} \omega_{\text {out }}^{2} q_{v_{2}}\left(l_{2}\right)-D_{\zeta_{2}} q_{v_{2}}^{\prime \prime}(0)+\omega_{\text {out }}^{2} \frac{I_{\zeta_{2}}^{2}}{m_{2}} q_{v_{2}}^{\prime \prime}(0)- \\
-\frac{I_{\zeta_{2}} D_{\zeta_{2}}}{m_{2}}\left[q_{v_{2}}^{I V}\left(l_{2}\right)-q_{v_{2}}^{I V}(0)\right]=0, \\
\omega_{\text {out }}^{2} I_{\zeta_{2}} q_{v_{2}}^{\prime}\left(l_{2}\right)+D_{\zeta_{2}} q_{v_{2}}^{\prime \prime \prime}\left(l_{2}\right)=0, \\
q_{\Phi_{2}}(0)=-q_{w_{1}}^{\prime}\left(l_{1}\right), \\
q_{v_{2}}^{\prime \prime}(0)=q_{\varphi_{1}}\left(l_{1}\right), \\
\left.q_{v_{2}}^{\prime \prime}\right)=0, \\
q_{\Phi_{2}}^{\prime}\left(l_{2}\right)=0 .
\end{gathered}
$$

So, expansion of the terms of equation (82) using equations (75) - (78) and (83) - (86) leads to the conclusion that the left side of equation (82) is equal to zero, and therefore proves that the operators describing the out-of-plane motion are also self-adjoint.

Thus, for the L-shaped beam structure the linear modes are orthogonal to each other, and therefore the nonlinear system projected onto the linear mode shapes using the weighted residual 
approach results in a nonlinear system with discrete modal equations coupled only due to the nonlinear terms. Even in the single nonlinear beams with geometric nonlinearities the method, of projection of the nonlinear system of partial differential equations to the infinite base of mode shapes of the underlying linear system, has been used effectively to study nonlinear dynamics with localization phenomena, modal interactions etc (Balachandran, B., Nayfeh, A.H., in 1990a, Balachandran, B., Nayfeh, A.H., in 1990b, Nayfeh, A.H., Pai, F., in 2004 etc). Additionally, since the system is selfadjoint the formulation for dimension reduction through the center manifold theory as described in (Steindl and Troger 2008) can be applied. The truncation of the modes is significant and the dimension reduction method has to be carefully applied in order to study nonlinear dynamical phenomena of the infinite system maintaining the essential information to the finite system (Steindl and Troger 2008). This analysis paves the way for application of the method for dimension reduction and examination of the dynamics in a reduced order system.

\section{Results and Discussion}

The nonlinear equations of motion (24)-(31) of an L-shaped beam structure up to (and including) the second order nonlinearities have been derived, and with the associated boundary conditions explored in detail. It should be mentioned that the derived system of equations is valid to describe the motion of L-shaped beams comprised with beams having cross section properties varying with respect to the longitudinal direction. In the case of the linear equations of motion there are two possible uncoupled motions; in-plane motion and out-of-plane motion, however when considering the inclusion of second order nonlinearities it is shown that these motions are coupled. Therefore, all the possible motions in the model have to be considered to enable further examination of the nonlinear dynamics of the structure.

At this stage the validity of the derived equations is examined by comparing them with under certain assumptions the known forms of equations from the literature.

In the particular case where the local displacements of the secondary beam are zero (i.e. it assumes the characteristics of an undeformable body) then the equations take a form which describes the nonlinear dynamics of a cantilever beam with a tip mass. It becomes evident that by considering only the linear part of the equations of motion (24)-(31) the resulting equations of motion are the same as those reported in Georgiades et al. (2013a,b). Also the boundary conditions (36)-(43) and (52)(63) coincide with those reported in Georgiades et al. (2013a,b) and it should be also noted that it is necessary to consider some vanishing terms.

In the case where primary beam terms are neglected in the equations for the secondary beam, by setting all relative components to zero, then the derived equations of motion of the single primary and the single secondary beams should coincide. In this case, the global displacements in secondary beam are coinciding with the local displacements.

-Axial motion $\left(u_{2}\right)$

Then equation (27) takes the form:

$$
\begin{aligned}
& m_{2} \ddot{u}_{2}-\frac{\partial}{\partial s_{2}}\left[\lambda_{2}\left(1+u_{2}^{\prime}\right)\right]+c_{21} \dot{u}_{2}-\frac{\partial^{2}}{\partial s_{2}^{2}}\left[D_{\eta_{2}} W_{2}^{\prime} W_{2}^{\prime \prime}\right]-\frac{\partial^{2}}{\partial s_{2}^{2}}\left[D_{\zeta_{2}} V_{2}^{\prime} V_{2}^{\prime \prime}\right]+ \\
& +\frac{\partial}{\partial s_{2}}\left[D_{\eta_{2}} W_{2}^{\prime \prime^{2}}\right]+\frac{\partial}{\partial s_{2}}\left[D_{\zeta_{2}} V_{2}^{\prime \prime^{2}}\right]+\frac{\partial}{\partial s_{2}}\left[I_{\eta_{2}} \delta_{3} W_{2}^{\prime} \ddot{W}_{2}^{\prime}\right]+\frac{\partial}{\partial s_{2}}\left[I_{\zeta_{2}} \delta_{2} \ddot{V}_{2}^{\prime} V_{2}^{\prime}\right]=0
\end{aligned}
$$

and this is clearly the same as equation (24) which describes the axial motion of the primary beam. It should be mentioned that both equations are identical to those derived by Arafat (1999) who also used three Euler angles in the derivation of the equations of motion of a single beam.

-In-plane bending motion $\left(W_{2}\right)$

In this case equation (29) takes the form, 


$$
\begin{gathered}
m_{2} \ddot{W}_{2}-\frac{\partial}{\partial s_{2}}\left[I_{\zeta_{2}} \delta_{2}\left(\dot{\phi}_{2} \dot{V}_{2}^{\prime}+\phi_{2} \ddot{V}_{2}^{\prime}\right)\right]+\frac{\partial}{\partial s_{2}}\left[I_{\eta_{2}} \delta_{3}\left(-\ddot{W}_{2}^{\prime}+\dot{\phi}_{2} \dot{V}_{2}^{\prime}+\phi_{2} \ddot{V}_{2}^{\prime}\right)\right]+ \\
+\frac{\partial}{\partial s_{2}}\left[I_{\xi_{2}} \dot{V}_{2}^{\prime} \dot{\phi}_{2}\right]-\frac{\partial}{\partial s_{2}}\left[\lambda_{2}\left(W_{2}^{\prime}\right)\right]-\frac{\partial^{2}}{\partial s_{2}^{2}}\left[D_{\eta_{2}}\left(-W_{2}^{\prime \prime}+\phi_{2} V_{2}^{\prime \prime}\right)\right]+ \\
+\frac{\partial^{2}}{\partial s_{2}^{2}}\left[D_{\zeta_{2}} V_{2}^{\prime \prime} \phi_{2}\right]-\frac{\partial}{\partial s_{2}}\left[D_{\xi_{2}} \phi_{2}^{\prime} V_{2}^{\prime \prime}\right]-\tilde{q}_{z, 2}+c_{22} \dot{W}_{2}-\frac{\partial}{\partial s_{2}} M_{z, 2}=0,
\end{gathered}
$$

and it is found to be the same as equation (26) describing the out-of-plane bending motion of the primary beam. The coincident form of the in-plane bending motion equation with that of the out-ofplane bending motion is due to the configuration of the secondary beam whereby the same curvatures and angular velocities are used, leading to the same form of equations to describe the different motions of the structure. It should be mentioned that both coincide with those derived by Arafat (1999) using three Euler angles.

-Out-of-plane bending motion $\left(V_{2}\right)$

For this case equation (30) takes the form,

$$
\begin{gathered}
m_{2} \ddot{V}_{2}-\frac{\partial}{\partial s_{2}}\left[I_{\xi_{2}}\left(\dot{W}_{2}^{\prime} \dot{\phi}_{2}+W_{2}^{\prime} \ddot{\phi}_{2}\right)\right]-\frac{\partial}{\partial s_{2}}\left[I_{\zeta_{2}} \delta_{2}\left(\ddot{V}_{2}^{\prime}+\ddot{W}_{2}^{\prime} \phi_{2}+\dot{\phi}_{2} \dot{W}_{2}^{\prime}\right)\right]+ \\
+\frac{\partial}{\partial s_{2}}\left[I_{\eta_{2}} \delta_{3}\left(\dot{\phi}_{2} \dot{W}_{2}^{\prime}+\phi_{2} \ddot{W}_{2}^{\prime}\right)\right]-\frac{\partial}{\partial s_{2}}\left[\lambda_{2} V_{2}^{\prime}\right]+\frac{\partial^{2}}{\partial s_{2}^{2}}\left[D_{\xi_{2}} W_{2}^{\prime} \phi_{2}^{\prime}\right]- \\
-\frac{\partial^{2}}{\partial s_{2}^{2}}\left[D_{\eta_{2}} \phi_{2} W_{2}^{\prime \prime}\right]+\frac{\partial^{2}}{\partial s_{2}^{2}}\left[D_{\zeta_{2}}\left(V_{2}^{\prime \prime}+\phi_{2} W_{2}^{\prime \prime}\right)\right]-\tilde{q}_{y, 2}+c_{23} \dot{V}_{2}+\frac{\partial}{\partial s_{2}} M_{y, 2}=0,
\end{gathered}
$$

and it proves to be the same as equation (25) which describes the in-plane bending motion of the primary beam. Both equations are identical to those derived by Arafat (1999) using three Euler angles.

-Torsional motion $\left(\phi_{2}\right)$

This is described by,

$$
\begin{gathered}
I_{\xi_{2}}\left(\ddot{\phi}_{2}+\ddot{V}_{2}^{\prime} W_{2}^{\prime}+\dot{V}_{2}^{\prime} \dot{W}_{2}^{\prime}\right)+I_{\eta_{2}} \delta_{3} \dot{V}_{2}^{\prime} \dot{W}_{2}^{\prime}- \\
-I_{\zeta_{2}} \delta_{2} \dot{V}_{2}^{\prime} \dot{W}_{2}^{\prime}-\frac{\partial}{\partial s_{2}}\left[D_{\xi_{2}}\left(\phi_{2}^{\prime}+V_{2}^{\prime \prime} W_{2}^{\prime}\right)\right]-D_{\eta_{2}} W_{2}^{\prime \prime} V_{2}^{\prime \prime}+D_{\zeta_{2}} W_{2}^{\prime \prime} V_{2}^{\prime \prime}-\widetilde{M}_{x, 2}+c_{24} \dot{\phi}_{2}=0
\end{gathered}
$$

and it can be seen that this is the same as equation (27) which describes the torsional motion of the primary beam. Once again there is agreement with those derived by Arafat (1999) using three Euler angles.

At this stage, it will be discussed the consideration of out-of-plane motions, whereas it should be mentioned that in the case of excitation of the out-of-plane motions in the L-Shaped beam structure, this is the only nonlinear model existing in the literature that describes these motionsIt is worth noting that out-of-plane motion can be excited with similar amplitudes as the in-plane motion when the flexibilities of the beams are comparable in both directions. At this stage, it will be discussed the consideration of out-of-plane motions, whereas it should be mentioned that in the case of excitation of the out-of-plane motions in the L-Shaped beam structure, this is the only nonlinear model existing in the literature that describes these motions. It is worth noting that out-of-plane motion can be excited with similar amplitudes as the in-plane motion when the flexibilities of the beams are comparable in both directions. Also it is examined the significance of the out-of-plane motion in inplane motion focusing on equation (25) which describes the in-plane bending motion. Considering that 
the out-of-plane motion is not excited and also the explicit form of Lagrange multiplier up to $1^{\text {st }}$ order is given by equation (C.4), one gets:

$$
\begin{gathered}
m_{1} \ddot{v}_{1}-\frac{\partial}{\partial s_{1}}\left[I_{\xi_{1}}\left(\dot{w}_{1}^{\prime} \dot{\varphi}_{1}+w_{1}^{\prime} \ddot{\varphi}_{1}\right)\right]+\frac{\partial}{\partial s_{1}}\left[\delta_{1} I_{\eta_{1}}\left(\dot{\varphi}_{1} \dot{w}_{1}^{\prime}+\varphi_{1} \ddot{w}_{1}^{\prime}\right)\right]- \\
-\frac{\partial}{\partial s_{1}}\left[I_{\zeta_{1}} \delta_{3}\left(\ddot{v}_{1}^{\prime}+\ddot{w}_{1}^{\prime} \varphi_{1}+\dot{\varphi}_{1} \dot{w}_{1}^{\prime}\right)\right]+\frac{\partial}{\partial s_{1}}\left[I_{\eta_{2}} \delta_{3} \ddot{W}_{2}^{\prime}(0, t) v_{1}^{\prime}-D_{\eta_{2}} W_{2}^{\prime \prime \prime}(0, t) v_{1}^{\prime}\right]+ \\
+\frac{\partial^{2}}{\partial s_{1}^{2}}\left[D_{\xi_{1}} w_{1}^{\prime} \varphi_{1}^{\prime}\right]-\frac{\partial^{2}}{\partial s_{1}^{2}}\left[D_{\eta_{1}} \varphi_{1} w_{1}^{\prime \prime}\right]+ \\
+\frac{\partial^{2}}{\partial s_{1}^{2}}\left[D_{\zeta_{1}}\left(v_{1}^{\prime \prime}+\varphi_{1} w_{1}^{\prime \prime}\right)\right]-\tilde{q}_{y, 1}+c_{12} \dot{v}_{1}+\frac{\partial}{\partial s_{1}} M_{y, 1}=0 .
\end{gathered}
$$

It is then considered an L-shaped beam structure with the same primary and secondary beam (also the same orientation) and having orthogonal section and length $\mathrm{L}=1 \mathrm{~m}$.

The beams have their 'width' $b$ (in the $Z_{1}$ direction for the primary beam and in the $Y_{2}$ direction for the secondary beam) and 'height' $h$ (in the $Y_{1}$ direction for the primary beam and in the $Z_{2}$ for the secondary beam) and their properties are constant with respect to the longitudinal direction.

It is introduced the following ratio for width and height:

$$
\frac{h}{b}=a \Leftrightarrow h=b \cdot a
$$

The stiffness and inertia coefficients are given in Georgiades et al. (2013b) as follows,

$$
\begin{array}{cc}
m_{1}=m_{2}=\rho b h=\rho a b^{2}, & I_{\eta_{1}}=\rho \frac{h \cdot b^{3}}{12}=\rho \frac{a \cdot b^{4}}{12}, \\
I_{\zeta_{1}}=\rho \frac{b \cdot h^{3}}{12}=\rho \frac{a^{3} \cdot b^{4}}{12}=a^{2} I_{\eta_{1}}, & I_{\xi_{1}}=\rho\left(\frac{a \cdot b^{4}+a^{3} \cdot b^{4}}{12}\right)=\left(1+a^{2}\right) I_{\eta_{1}}, \\
D_{\eta_{1}}=E \frac{h \cdot b^{3}}{12}=E \frac{a \cdot b^{4}}{12}, & D_{\zeta_{1}}=E \frac{b \cdot h^{3}}{12}=E \frac{a^{3} \cdot b^{4}}{12}=a^{2} D_{\eta_{1}}, \\
D_{\xi_{1}}=G_{12} \frac{1}{3} b \cdot h^{3}\left\{1-\frac{192 h}{\pi^{5} b} \sum_{n=1,3, \ldots}^{\infty}\left[\frac{1}{n^{5}} \tanh \left(\frac{n \pi b}{2 h}\right)\right]\right\}, \\
I_{\eta_{2}}=\rho \frac{b \cdot h^{3}}{12}=\rho \frac{a^{3} \cdot b^{4}}{12}=a^{2} I_{\eta_{1}}, & I_{\zeta_{2}}=\rho \frac{h \cdot b^{3}}{12}=\rho \frac{a \cdot b^{4}}{12}=I_{\eta_{1}}, \\
D_{\zeta_{2}}=E \frac{h \cdot b^{3}}{12}=D_{\eta_{1}}, & D_{\eta_{2}}=E \frac{b \cdot h^{3}}{12}=a^{2} D_{\eta_{1}} \\
D_{\xi_{2}}=G_{12} \frac{1}{3} b \cdot h^{3}\left\{1-\frac{192 h}{\pi^{5} b} \sum_{n=1,3, \ldots}^{\infty}\left[\frac{1}{n^{5}} \tanh \left(\frac{n \pi b}{2 h}\right)\right]\right\} .
\end{array}
$$

Considering the definitions of coefficients (eq. 93a-1), equation (91) takes the form,

$$
\begin{gathered}
m_{1} \ddot{v}_{1}-\left(1+a^{2}\right) I_{\eta_{1}} \frac{\partial}{\partial s_{1}}\left[\dot{w}_{1}^{\prime} \dot{\varphi}_{1}+w_{1}^{\prime} \ddot{\varphi}_{1}\right]-\delta_{1} I_{\eta_{1}} \frac{\partial}{\partial s_{1}}\left[-\dot{\varphi}_{1} \dot{w}_{1}^{\prime}-\varphi_{1} \ddot{w}_{1}^{\prime}\right]- \\
-a^{2} I_{\eta_{1}} \delta_{3} \frac{\partial}{\partial s_{1}}\left[\ddot{v}_{1}^{\prime}+\ddot{w}_{1}^{\prime} \varphi_{1}+\dot{\varphi}_{1} \dot{w}_{1}^{\prime}\right]+a^{2} I_{\eta_{1}} \delta_{3} \ddot{W}_{2}^{\prime}(0, t) v_{1}^{\prime \prime}-a^{2} D_{\eta_{1}} W_{2}^{\prime \prime \prime}(0, t) v_{1}^{\prime \prime}+ \\
+\boldsymbol{D}_{\xi_{1}} \frac{\partial^{2}}{\partial s_{1}^{2}}\left[\boldsymbol{w}_{1}^{\prime} \boldsymbol{\varphi}_{1}^{\prime}\right]+\left(\mathbf{1}-\boldsymbol{a}^{2}\right) \boldsymbol{D}_{\eta_{1}} \frac{\partial^{2}}{\partial s_{1}^{2}}\left[\boldsymbol{\varphi}_{1} \boldsymbol{w}_{1}^{\prime \prime}\right]+ \\
+\boldsymbol{a}^{2} \boldsymbol{D}_{\eta_{1}} \frac{\partial^{2}}{\partial s_{1}^{2}}\left[\boldsymbol{v}_{1}^{\prime \prime}\right]-\tilde{q}_{y, 1}+c_{12} \dot{v}_{1}+\frac{\partial}{\partial s_{1}} M_{y, 1}=0
\end{gathered}
$$


The rotary inertia coupling terms in dimensionless equation (94) contain 2 nd order nonlinear terms. Also the rotary inertia terms after nondimsionalisation of equation (94) are of even higher order, and in most cases are neglected (Nayfeh and Pai 2004). Therefore these terms cannot form the basis of our examination in terms of the significance of the coupling terms of in-plane with out-ofplane motion.

The key terms (in bold) in this equation which couples the in-plane motion with the out-ofplane motion are explored further on. It should be mentioned that in equation (94), which is of 2nd order and describes the motion $v_{1}$, the only nonlinear stiffness terms are those that couple the in-plane with out-of-plane motion, and these coupling terms will become even more significant when one incorporates 3 rd order terms in $v_{1}$.

It should be mentioned that all the stiffness terms inside the brackets are associated with the second order derivatives and in case of nondimensionalisation the stiffness coefficients will play a significant role.

It will be examined three cases of cross-sections of beams made of aluminium $(\mathrm{E}=70 \mathrm{MPa}$ and $\left.\mathrm{G}_{12}=26.32 \mathrm{MPa}\right)$ and using equations $(92,93 \mathrm{e}, \mathrm{g})$ the calculated stiffness coefficients are shown in Table 1. It should be mentioned that similar results can be obtained in the case of steel structures.

Table 1. Stiffness coefficients.

\begin{tabular}{|c|c|c|c|c|c|c|}
\hline$a$ & $a^{2}$ & $\mathrm{~b}(\mathrm{~m})$ & $\mathrm{h}(\mathrm{m})$ & $D_{\eta_{1}}\left(\mathrm{~Pa} \mathrm{~m}^{4}\right)$ & $D_{\xi_{1}}\left(\mathrm{~Pa} \mathrm{~m}^{4}\right)$ & $a^{2} D_{\eta_{1}}\left(\mathrm{~Pa} \mathrm{~m}^{4}\right)$ \\
\hline 1 & 1 & 0.05 & 0.05 & 36458 & 23125 & 36458 \\
\hline 0.1 & 0.01 & 0.05 & 0.005 & 3645.8 & 51.377 & 36.45 \\
\hline 10 & 100 & 0.005 & 0.05 & 36.458 & 51.377 & 3645.8 \\
\hline
\end{tabular}

1) $a=1$, therefore the stiffness terms in equation (94) are taking the form,

$$
\boldsymbol{D}_{\boldsymbol{\eta}_{1}} v_{1}^{I V}+\boldsymbol{D}_{\xi_{1}} \frac{\partial^{2}}{\partial s_{1}^{2}}\left[w_{1}^{\prime} \varphi_{1}^{\prime}\right]
$$

whereas based on Table 1 the torsional rigidity has the same order of magnitude with the bending stiffness, therefore the coupling terms are significant.

2) $a<1 \Leftrightarrow-\left(1-a^{2}\right) \approx-1$, and the stiffness terms in equation (94) take the form,

$$
D_{\xi_{1}} \frac{\partial^{2}}{\partial s_{1}^{2}}\left[w_{1}^{\prime} \varphi_{1}^{\prime}\right]+D_{\eta_{1}} \frac{\partial^{2}}{\partial s_{1}^{2}}\left[\varphi_{1} w_{1}^{\prime \prime}\right]+\varepsilon^{2} D_{\eta_{1}} v_{1}^{I V},
$$

in this case the stiffness of the linear term has very small value $\left(\varepsilon^{2}\right)$ and therefore the out-of-plane motions are significant. Also on the basis of the results in Table 1 the torsional rigidity is seen to be higher than the linear bending stiffness.

3) $a>1 \Leftrightarrow-\left(1-a^{2}\right) \approx a^{2}$

$$
D_{\xi_{1}} \frac{\partial^{2}}{\partial s_{1}^{2}}\left[w_{1}^{\prime} \varphi_{1}^{\prime}\right]-\boldsymbol{a}^{2} \boldsymbol{D}_{\boldsymbol{\eta}_{\mathbf{1}}} \frac{\partial^{2}}{\partial s_{1}^{2}}\left[\varphi_{1} w_{1}^{\prime \prime}\right]+\boldsymbol{a}^{2} \boldsymbol{D}_{\boldsymbol{\eta}_{\mathbf{1}}} v_{1}^{I V}
$$

in this case the bending stiffnesses of the linear term and the nonlinear term have comparable values and thus the out-of-plane motions are significant.

Therefore, in all cases the coupling of in-plane with out-of-plane motion is essential, and since there are no exemptions it is sufficient the examination of the coupling of just this equation of in-phase motion with out-of-phase motion of primary beam. It is concluded, that the out-of-plane motions have to be considered even in examining the in-plane motion even in 2 nd order approximation. It should be 
commented that even in single beams restricted to 2nd order approximation, the out-of-plane motions are coupled with the in-plane motions as can be seen in the derived equations by Arafat in (1999) and Nayfeh and Pay in (2004) but it is not quantified the strong coupling as it is presented in the herein analysis. It should also be pointed out that the cases of beams with circular cross sections, corresponds to case 1 (with equal 'width' and 'height'), then the torsional rigidity is even higher since the Timoshenko warping correction factor is zero.

In order to extend the modelling up and including third order nonlinearities it has to be considered that the full third order terms in the curvatures and anguar velocities (eq. 1), following the work of Nayfeh and Pai in (2004), and in matrix A (equation 5) have to be included in higher order terms.

\section{Conclusions}

The nonlinear equations of motion were derived for an L-shaped beam structure using the global displacements of the secondary beam and the rotary inertia terms. In the linear case the equations for in-plane motion are uncoupled from those for out-of-plane motion. When considering the nonlinearities all the equations of motion are coupled and they all have to be considered in order to proceed with a subsequent examination of the nonlinear dynamics. This paper can serve as a benchmark for deriving a more complicated model that includes also the third order nonlinearities. Then the up to second order terms in the third order model should coincide with system presented in this paper. It has been shown in this paper that the linear system is self-adjoint and therefore the linear modal analysis results in orthogonal mode shapes. Therefore the derived equations of motion expressed in the form of linear global displacements can be discretised by projecting the dynamics onto the linear mode shapes and then continuing with nonlinear dynamic analysis. Moreover, by switching the parameter $\delta_{\mathrm{i}}$ the derived equations can be used to check the importance of the rotary inertia terms. In the next phase of this research the mathematical model will be reduced to a set of ordinary differential equations and then the dynamics of the coupled nonlinear system will be studied in comparison with those of the previously published linear model (Georgiades et al. 2013a,b). A low order reduced system of ordinary differential equations can be obtained which contains the most important nonlinear phenomena. Then, the inertia manifold theory will be applied in order to study and analyse the resulting dynamics (Steindl and Troger 2008).

Finally, it should be mentioned that the L-shaped beam structure is one of the physically simplest 'composite' structure and the work presented in this paper paves the way for nonlinear modelling and dynamic analysis of more complicated structures involving more structural components e.g. airplane or tree-like structures in mechanical installations, flexible buildings in civil engineering installations, and even the actual tree structures, considering geometric nonlinearities in the large deformation regime.

\section{Acknowledgements}

The author gratefully acknowledge the provision of funding from the European Union Seventh Framework Programme (FP7/2007-2013), FP7 - REGPOT - 2009 - 1, under grant agreement No:245479. The author would like also to thank Prof. Jerzy Warminski and Prof. Matthew Phillip Cartmell for their valuable comments on this article. The author would like to thanks the unknown reviewer for the valuable comments to improve the original manuscript.

\section{References}

Arafat, H.N., 1999, Nonlinear Response of Cantilever Beams, PhD Thesis Virginia Polytechnic Institute. 
Balachandran, B., Nayfeh, A.H., 1990a, Nonlinear motion of beam-mass structure, Nonlinear Dynamics, 1, 39-61.

Balachandran, B., Nayfeh, A.H., 1990b, Nonlinear Oscillations of a harmonically excited composite structure, Composite Structures, 16, 323-339.

Bolotin, V.V., 1964, The Dynamic Stability of Elastic Systems, San Fransisco, Holden-Day.

Bux, S. L., Roberts, J. W., 1986, Non-Linear Vibratory Interactions in Systems of Coupled Beams, Journal of Sound and Vibration, 104, 497-520.

Cartmell, M.P., Roberts, J.W., 1987, Simultaneous combination resonances in a parametrically excited cantilever beam, Strain, 23, 117-126.

Haddow, A.G., Barr, A.D.S., Mook, D.T., 1984, Theoretical and Experimental Study of Modal Interaction in a Two-Degree-of-Freedom, Structure, Journal of Sound and Vibration, 97, 451-473.

Georgiades, F., Warminski, J., Cartmell, P., M., 2013a, 'Towards Linear Modal Analysis for an LShaped Beam: Equations of Motion', Mechanics Research Communications, 47, pp 50-60, doi: 10.1016/j.mechrescom.2012.11.005.

Georgiades, F., Warminski, J., Cartmell, P., M., 2013b, 'Linear Modal Analysis of L-Shaped beam Structures', Mechanical Systems and Signal Processing, 38, pp 312-332, doi:10.1016/j.ymssp.2012.12.006.

Nayfeh, A.H., 1981, Introduction to Perturbation Techniques, Wiley-Intersciences, NewYork. Nayfeh, A.H., Pai, F., 2004, Linear and Nonlinear Structural Mechanics, first edition, John Willey and sons, New Jersey.

Ozonato, N., Nagai, K.-I., Maruyama, S., Yamaguchi, T., 2012, Chaotic Vibrations of A Post-Buckled L-Shape Beam with an Axial Constraint, Nonlinear Dynamics, 67, 2363-2379.

Roberts, J.W., Cartmell, M.P., 1984, Forced vibration of a beam system with autoparametric coupling effects, Strain, 20, 123-131.

Steindl, A., Troger, H., 2008, Dimension Reduction: A Key Concept in Dynamics, ENOC-2008, Saint Petersburg, Russia.

Warminski, J., Cartmell, M.P., Bochenski, M., Ivanov, I., 2008, Analytical and experimental investigations of an autoparametric beam structure, Journal of Sound and Vibration, 315, 486-508.

\section{Appendix-A}

Considering the transformations given in eq.(11-13) the angular velocities and curvatures of the secondary beam, equations ( $2 \mathrm{a}-\mathrm{f})$ take the form,

$$
\begin{gathered}
\rho_{\xi_{2}}=\left\{\rho_{\xi_{2}}^{(1)}\right\}+\left\{\rho_{\xi_{2}}^{(2)}\right\}+\left\{\rho_{\xi_{2}}^{(3 *)}\right\}=\left\{\phi_{2}^{\prime}\right\}+\left\{V_{2}^{\prime \prime} W_{2}^{\prime}+v_{1 C}^{\prime} V_{2}^{\prime \prime}\right\}+\{0\}, \\
\rho_{\eta_{2}}=\left\{\rho_{\eta_{2}}^{(1)}\right\}+\left\{\rho_{\eta_{2}}^{(2)}\right\}+\left\{\rho_{\eta_{2}}^{(3 *)}\right\}=\left\{-W_{2}^{\prime \prime}\right\}+\left\{\phi_{2} V_{2}^{\prime \prime}+w_{1 C}^{\prime} V_{2}^{\prime \prime}\right\}+\left\{u_{2}^{\prime \prime} W_{2}^{\prime}+u_{2}^{\prime \prime} v_{1 c}^{\prime}+u_{2}^{\prime} W_{2}^{\prime \prime}\right\}, \\
\rho_{\zeta_{2}}=\left\{\rho_{\zeta_{2}}^{(1)}\right\}+\left\{\rho_{\zeta_{2}}^{(2)}\right\}+\left\{\rho_{\zeta_{2}}^{(3 *)}\right\}=\left\{V_{2}^{\prime \prime}\right\}+\left\{\phi_{2} W_{2}^{\prime \prime}+w_{1 C}^{\prime} W_{2}^{\prime \prime}\right\}+\left\{-u_{2}^{\prime \prime} V_{2}^{\prime}+u_{2}^{\prime \prime} \varphi_{1 C}-u_{2}^{\prime} V_{2}^{\prime \prime}\right\},
\end{gathered}
$$




$$
\begin{gathered}
\omega_{\xi_{2}}=\left\{\omega_{\xi_{2}}^{(1)}\right\}+\left\{\omega_{\xi_{2}}^{(2)}\right\}+\left\{\omega_{\xi_{2}}^{(3 *)}\right\}=\left\{\dot{\phi}_{2}\right\}+\left\{\dot{V}_{2}^{\prime} W_{2}^{\prime}+\dot{V}_{2}^{\prime} v_{1 C}^{\prime}-\dot{\varphi}_{1 c} W_{2}^{\prime}-\dot{\varphi}_{1 c} v_{1 C}^{\prime}+\delta_{1} \varphi_{1 c} \dot{v}_{1 c}^{\prime}\right\}+ \\
+\left\{\delta_{1} \dot{u}_{1 c}^{\prime} w_{1 c}^{\prime}+\delta_{1} u_{1 c}^{\prime} \dot{w}_{1 c}^{\prime}\right\}, \\
\omega_{\eta_{2}}=\left\{\omega_{\eta_{2}}^{(1)}\right\}+\left\{\omega_{\eta_{2}}^{(2)}\right\}+\left\{\omega_{\eta_{2}}^{(3 *)}\right\}=\left\{\delta_{3}\left(-\dot{W}_{2}^{\prime}\right)\right\}+\left\{\delta _ { 3 } \left(\phi_{2} \dot{V}_{2}^{\prime}+w_{1 c}^{\prime} \dot{V}_{2}^{\prime}-\phi_{2} \dot{\varphi}_{1 c}-\right.\right. \\
\left.-w_{1 c}^{\prime} \dot{\varphi}_{1 c}+\varphi_{1 c} \dot{w}_{1 c}^{\prime}\right\}+\left\{\delta_{3}\left(\dot{u}_{2}^{\prime} W_{2}^{\prime}+\dot{u}_{2}^{\prime} v_{1 c}^{\prime}+u_{2}^{\prime} \dot{W}_{2}^{\prime}+u_{2}^{\prime} \dot{v}_{1 c}^{\prime}\right)\right\}, \\
\omega_{\zeta_{2}}=\left\{\omega_{\zeta_{2}}^{(1)}\right\}+\left\{\omega_{\zeta_{2}}^{(2)}\right\}+\left\{\omega_{\zeta_{2}}^{(3 *)}\right\}=\left\{\delta_{2}\left(\dot{V}_{2}^{\prime}\right)\right\}+\left\{\delta_{2}\left(\phi_{2} \dot{W}_{2}^{\prime}+\dot{v}_{1 c}^{\prime} \phi_{2}+\dot{W}_{2}^{\prime} w_{1 c}^{\prime}+2 \dot{v}_{1 c}^{\prime} w_{1 c}^{\prime}\right)\right\}+ \\
+\left\{\delta_{2}\left(-\dot{u}_{2}^{\prime} V_{2}^{\prime}+\dot{u}_{2}^{\prime} \varphi_{1 c}-u_{2}^{\prime} \dot{V}_{2}^{\prime}+u_{2}^{\prime} \dot{\varphi}_{1 c}\right)\right\} .
\end{gathered}
$$

Similarly, the translational velocities of the secondary beam in equation (9), using the transformations given in equations (11-13) are defined by,

$$
\begin{gathered}
V_{x_{2}}=\left\{V_{x_{2}}^{(1)}\right\}+\left\{V_{x_{2}}^{(2)}\right\}+\left\{V_{x_{2}}^{(3 *)}\right\}=\left\{\dot{v}_{1 c}\right\}+\left\{\dot{u}_{2}+\dot{v}_{1 c}^{\prime} W_{2}+s_{2} \dot{v}_{1 c}^{\prime} v_{1 c}^{\prime}-\dot{\varphi}_{1 c} V_{2}+s_{2} \varphi_{1 c} \dot{\varphi}_{1 c}+\right. \\
\left.+\varphi_{1 c} \dot{w}_{1 c}+\dot{\varphi}_{1 c} w_{1 c}\right\}+\left\{-v_{1 c}^{\prime} \dot{u}_{1 c}-\dot{v}_{1 c}^{\prime} u_{1 c}\right\} \\
V_{y_{2}}=\left\{V_{y_{2}}^{(1)}\right\}+\left\{V_{y_{2}}^{(2)}\right\}+\left\{V_{y_{2}}^{(3 *)}\right\}=\left\{\dot{V}_{2}\right\}+\left\{-\varphi_{1 c} \dot{v}_{1 c}+W_{2} \dot{w}_{1 c}^{\prime}+s_{2} \dot{v}_{1 c}^{\prime} w_{1 c}^{\prime}+s_{2} v_{1 c}^{\prime} \dot{w}_{1 c}^{\prime}\right\}+ \\
+\left\{-w_{1 c}^{\prime} \dot{u}_{1 c}+u_{2} \dot{\varphi}_{1 c}-u_{1 c} \dot{w}_{1 c}^{\prime}\right\} \\
V_{z_{2}}=\left\{V_{z_{2}}^{(1)}\right\}+\left\{V_{z_{2}}^{(2)}\right\}+\left\{V_{z_{2}}^{(3 *)}\right\}=\left\{\dot{W}_{2}\right\}+\left\{v_{1 c}^{\prime} \dot{v}_{1 c}+w_{1 c}^{\prime} \dot{w}_{1 c}-V_{2} \dot{w}_{1 c}^{\prime}+w_{1 c} \dot{w}_{1 c}^{\prime}\right\}+ \\
+\left\{-u_{2} \dot{v}_{1 c}^{\prime}\right\} .
\end{gathered}
$$

\section{Appendix-B}

In this appendix all the terms of equation (22) are determined explicitly. Initially it can be obtained the terms for the equations of motion of the:

-Primary beam,

a) Axial motion equation terms $\left(\delta u_{1}\right)$,

$$
\begin{gathered}
\frac{\partial^{2} h_{T_{1}}}{\partial \dot{u}_{1} \partial t}=2 m_{1} \frac{\partial}{\partial t}\left(\frac{\partial V_{x_{1}}}{\partial \dot{u}_{1}} V_{x_{1}}\right)=2 m_{1} \ddot{u}_{1} \\
\frac{\partial^{2} h_{F_{1}}}{\partial u_{1}^{\prime} \partial s_{1}}=-2 \frac{\partial}{\partial s_{1}}\left[\lambda_{1}\left(1+u_{1}^{\prime}\right)\right] \\
\frac{\partial^{3} h_{T_{1}}}{\partial \dot{u}_{1}^{\prime} \partial s_{1} \partial t}=2 \frac{\partial^{2}}{\partial s_{1} \partial t}\left[I_{\zeta_{1}} \frac{\partial \omega_{\zeta_{1}}^{(3)}}{\partial \dot{u}_{1}^{\prime}} \omega_{\zeta_{1}}^{(1)}+I_{\eta_{1}} \frac{\partial \omega_{\eta_{1}}^{(3)}}{\partial \dot{u}_{1}^{\prime}}\left(\omega_{\eta_{1}}^{(1)}\right)\right]= \\
=-2 \frac{\partial}{\partial s_{1}}\left\{I_{\zeta_{1}} \delta_{3}\left[\left(\dot{v}_{1}^{\prime}\right)^{2}+v_{1}^{\prime} \ddot{v}_{1}^{\prime}\right]\right\}+2 \frac{\partial}{\partial s_{1}}\left\{I_{\eta_{1}} \delta_{1}\left[\left(\dot{w}_{1}^{\prime}\right)^{2}+w_{1}^{\prime} \ddot{w}_{1}^{\prime}\right]\right\} \\
\frac{\partial^{2} h_{T_{1}}}{\partial u_{1}^{\prime} \partial s_{1}}=2 \frac{\partial}{\partial s_{1}}\left[I_{\zeta_{1}} \frac{\partial \omega_{\zeta_{1}}^{(3)}}{\partial u_{1}^{\prime}} \omega_{\zeta_{1}}^{(1)}+I_{\eta_{1}} \frac{\partial \omega_{\eta_{1}}^{(3)}}{\partial u_{1}^{\prime}} \omega_{\eta_{1}}^{(1)}\right]=-2 \frac{\partial}{\partial s_{1}}\left[I_{\zeta_{1}} \delta_{3}\left(\dot{v}_{1}^{\prime}\right)^{2}\right]-2 \frac{\partial}{\partial s_{1}}\left[I_{\eta_{1}} \delta_{1}\left(\dot{w}_{1}^{\prime}\right)^{2}\right], \\
\frac{\partial^{3} h_{U_{1}}}{\partial u_{1}^{\prime \prime} \partial s_{1}^{2}}=2 \frac{\partial^{2}}{\partial s_{1}^{2}}\left[D_{\eta_{1}} \frac{\partial \rho_{\eta_{1}}^{(3)}}{\partial u_{1}^{\prime \prime}} \rho_{\eta_{1}}^{(1)}+D_{\zeta_{1}} \frac{\partial \rho_{\zeta_{1}}^{(3)}}{\partial u_{1}^{\prime \prime}} \rho_{\zeta_{1}}^{(1)}\right]=-2 \frac{\partial^{2}}{\partial s_{1}^{2}}\left[D_{\eta_{1}} w_{1}^{\prime} w_{1}^{\prime \prime}\right]-2 \frac{\partial^{2}}{\partial s_{1}^{2}}\left[D_{\zeta_{1}} v_{1}^{\prime} v_{1}^{\prime \prime}\right]
\end{gathered}
$$




$$
\frac{\partial^{2} h_{U_{1}}}{\partial u_{1}^{\prime} \partial s_{1}}=2 \frac{\partial}{\partial s_{1}}\left[D_{\eta_{1}} \frac{\partial \rho_{\eta_{1}}^{(3)}}{\partial u_{1}^{\prime}} \rho_{\eta_{1}}^{(1)}+D_{\zeta_{1}} \frac{\partial \rho_{\zeta_{1}}^{(3)}}{\partial u_{1}^{\prime}} \rho_{\zeta_{1}}^{(1)}\right]=-2 \frac{\partial}{\partial s_{1}}\left[D_{\eta_{1}}\left(w_{1}^{\prime \prime}\right)^{2}\right]-2 \frac{\partial}{\partial s_{1}}\left[D_{\zeta_{1}}\left(v_{1}^{\prime \prime}\right)^{2}\right]
$$

b) In-plane bending equation terms $\left(\delta v_{1}\right)$,

$$
\begin{gathered}
\frac{\partial^{2} h_{T_{1}}}{\partial \dot{v}_{1} \partial t}=2 m_{1} \frac{\partial}{\partial t}\left(\frac{\partial V_{y_{1}}}{\partial \dot{v}_{1}} V_{y_{1}}\right)=2 m_{1} \ddot{v}_{1} \\
\frac{\partial^{3} h_{T_{1}}}{\partial \dot{v}_{1}^{\prime} \partial s_{1} \partial t}=2 \frac{\partial^{2}}{\partial s_{1} \partial t}\left[I_{\xi_{1}} \frac{\partial \omega_{\xi_{1}}^{(2)}}{\partial \dot{v}_{1}^{\prime}}\left(\omega_{\xi_{1}}^{(1)}\right)+I_{\eta_{1}} \frac{\partial \omega_{\eta_{1}}^{(2)}}{\partial \dot{v}_{1}^{\prime}}\left(\omega_{\eta_{1}}^{(1)}\right)+I_{\zeta_{1}} \frac{\partial \omega_{\zeta_{1}}^{(1)}}{\partial \dot{v}_{1}^{\prime}}\left(\omega_{\zeta_{1}}^{(1)}+\omega_{\zeta_{1}}^{(2)}\right)\right]= \\
=2 \frac{\partial}{\partial s_{1}}\left[I_{\xi_{1}}\left(\dot{w}_{1}^{\prime} \dot{\varphi}_{1}+w_{1}^{\prime} \ddot{\varphi}_{1}\right)\right]-2 \frac{\partial}{\partial s_{1}}\left[I_{\eta_{1}} \delta_{1}\left(\dot{\varphi}_{1} \dot{w}_{1}^{\prime}+\varphi_{1} \ddot{w}_{1}^{\prime}\right)\right]+2 \frac{\partial}{\partial s_{1}}\left[I_{\zeta_{1}} \delta_{3}\left(\ddot{v}_{1}^{\prime}+\ddot{w}_{1}^{\prime} \varphi_{1}+\dot{\varphi}_{1} \dot{w}_{1}^{\prime}\right)\right], \\
\frac{\partial^{3} h_{U_{1}}}{\partial v_{1}^{\prime \prime} \partial s_{1}^{2}}=2 \frac{\partial^{2}}{\partial s_{1}^{2}}\left[D_{\xi_{1}} \frac{\partial \rho_{\xi_{1}}^{(2)}}{\partial v_{1}^{\prime \prime}}\left(\rho_{\xi_{1}}^{(1)}\right)+D_{\eta_{1}} \frac{\partial \rho_{\eta_{1}}^{(2)}}{\partial v_{1}^{\prime \prime}}\left(\rho_{\eta_{1}}^{(1)}+\rho_{\eta_{1}}^{(2)}\right)+D_{\zeta_{1}} \frac{\partial \rho_{\zeta_{1}}^{(1)}}{\partial v_{1}^{\prime \prime}}\left(\rho_{\zeta_{1}}^{(1)}+\rho_{\zeta_{1}}^{(2)}\right)\right]= \\
=2 \frac{\partial^{2}}{\partial s_{1}^{2}}\left[D_{\xi_{1}} w_{1}^{\prime} \varphi_{1}^{\prime}\right]-2 \frac{\partial^{2}}{\partial s_{1}^{2}}\left[D_{\eta_{1}} \varphi_{1} w_{1}^{\prime \prime}\right]+2 \frac{\partial^{2}}{\partial s_{1}^{2}}\left[D_{\zeta_{1}}\left(v_{1}^{\prime \prime}+\varphi_{1} w_{1}^{\prime \prime}\right)\right], \\
\frac{\partial^{2} h_{T_{1}}}{\partial v_{1}^{\prime} \partial s_{1}}=2 \frac{\partial}{\partial s_{1}}\left[I_{\zeta_{1}} \frac{\partial \omega_{\zeta_{1}}^{(3)}}{\partial v_{1}^{\prime}} \omega_{\zeta_{1}}^{(1)}\right]=-2 \frac{\partial}{\partial s_{1}}\left[I_{\zeta_{1}} \dot{u}_{1}^{\prime} \dot{v}_{1}^{\prime}\right], \text { H.O.T. } \\
\frac{\partial^{2} h_{U_{1}}}{\partial v_{1}^{\prime} \partial s_{1}}=-2 \frac{\partial}{\partial s_{1}}\left[D_{\zeta_{1}} u_{1}^{\prime \prime} v_{1}^{\prime \prime}\right], \text { H.O.T. }
\end{gathered}
$$

whereas, H.O.T., means that this term is of higher order ( $3^{\text {rd }}$ order) and will be neglected from now on.

c) Out-of-plane bending equation terms $\left(\delta w_{1}\right)$,

$$
\begin{gathered}
\frac{\partial^{2} h_{T_{1}}}{\partial \dot{w}_{1} \partial t}=2 m_{1} \frac{\partial}{\partial t}\left(\frac{\partial V_{z_{1}}}{\partial \dot{w}_{1}} V_{z_{1}}\right)=2 m_{1} \ddot{w}_{1}, \\
\frac{\partial^{3} h_{T_{1}}}{\partial \dot{w}_{1}^{\prime} \partial s_{1} \partial t}=2 \frac{\partial^{2}}{\partial s_{1} \partial t}\left[I_{\eta_{1}} \frac{\partial \omega_{\eta_{1}}^{(1)}}{\partial \dot{w}_{1}^{\prime}}\left(\omega_{\eta_{1}}^{(1)}+\omega_{\eta_{1}}^{(2)}\right)+I_{\zeta_{1}} \frac{\partial \omega_{\zeta_{1}}^{(2)}}{\partial \dot{w}_{1}^{\prime}}\left(\omega_{\zeta_{1}}^{(1)}\right)\right]= \\
=2 \frac{\partial}{\partial s_{1}}\left[I_{\eta_{1}} \delta_{1}\left(\ddot{w}_{1}^{\prime}-\dot{\varphi}_{1} \dot{v}_{1}^{\prime}-\varphi_{1} \ddot{v}_{1}^{\prime}\right)\right]+2 \frac{\partial}{\partial s_{1}}\left[I_{\zeta_{1}} \delta_{3}\left(\dot{\varphi}_{1} \dot{v}_{1}^{\prime}+\varphi_{1} \ddot{v}_{1}^{\prime}\right)\right] \\
\frac{\partial^{2} h_{T_{1}}}{\partial w_{1}^{\prime} \partial s_{1}}=2 \frac{\partial}{\partial s_{1}}\left[I_{\xi_{1}} \frac{\partial \omega_{\xi_{1}}^{(2)}}{\partial w_{1}^{\prime}}\left(\omega_{\xi_{1}}^{(1)}\right)\right]=2 \frac{\partial}{\partial s_{1}}\left[I_{\xi_{1}} \dot{v}_{1}^{\prime} \dot{\varphi}_{1}\right], \\
\frac{\partial^{3} h_{U_{1}}}{\partial w_{1}^{\prime \prime} \partial s_{1}^{2}}=2 \frac{\partial^{2}}{\partial s_{1}^{2}}\left[D_{\eta_{1}} \frac{\partial \rho_{\eta_{1}}^{(1)}}{\partial w_{1}^{\prime \prime}}\left(\rho_{\eta_{1}}^{(1)}+\rho_{\eta_{1}}^{(2)}\right)+D_{\zeta_{1}} \frac{\partial \rho_{\zeta_{1}}^{(2)}}{\partial w_{1}^{\prime \prime}}\left(\rho_{\zeta_{1}}^{(1)}+\rho_{\zeta_{1}}^{(2)}\right)\right]= \\
=2 \frac{\partial}{\partial s_{1}}\left[\lambda_{1} w_{1}^{\prime}\right], \\
\left.=D_{\eta_{1}}\left(w_{1}^{\prime \prime}-\varphi_{1} v_{1}^{\prime \prime}\right)\right]+2 \frac{\partial^{2}}{\partial s_{1}^{2}}\left[D_{\zeta_{1}} \varphi_{1} v_{1}^{\prime \prime}\right]
\end{gathered}
$$




$$
\frac{\partial^{2} h_{U_{1}}}{\partial w_{1}^{\prime} \partial s_{1}}=2 \frac{\partial}{\partial s_{1}}\left[D_{\xi_{1}} \frac{\partial \rho_{\xi_{1}}^{(2)}}{\partial w_{1}^{\prime}}\left(\rho_{\xi_{1}}^{(1)}\right)\right]=2 \frac{\partial}{\partial s_{1}}\left[D_{\xi_{1}} v_{1}^{\prime \prime} \varphi_{1}^{\prime}\right]
$$

d) Torsional motion equation terms $\left(\delta \varphi_{1}\right)$,

$$
\begin{gathered}
\frac{\partial^{2} h_{T_{1}}}{\partial \dot{\varphi}_{1} \partial t}=2 \frac{\partial}{\partial t}\left[I_{\xi_{1}} \frac{\partial \omega_{\xi_{1}}^{(1)}}{\partial \dot{\varphi}_{1}}\left(\omega_{\xi_{1}}^{(1)}+\omega_{\xi_{1}}^{(2)}\right)\right]=2 I_{\xi_{1}}\left(\ddot{\varphi}_{1}+\ddot{v}_{1}^{\prime} w_{1}^{\prime}+\dot{v}_{1}^{\prime} \dot{w}_{1}^{\prime}\right), \\
\frac{\partial h_{T_{1}}}{\partial \varphi_{1}}=2\left[I_{\eta_{1}} \frac{\partial \omega_{\eta_{1}}^{(2)}}{\partial \varphi_{1}}\left(\omega_{\eta_{1}}^{(1)}\right)+I_{\zeta_{1}} \frac{\partial \omega_{\zeta_{1}}^{(2)}}{\partial \varphi_{1}}\left(\omega_{\zeta_{1}}^{(1)}\right)\right]=-2 \delta_{1} I_{\eta_{1}} \dot{v}_{1}^{\prime} \dot{w}_{1}^{\prime}+2 \delta_{3} I_{\zeta_{1}} \dot{v}_{1}^{\prime} \dot{w}_{1}^{\prime} \\
\frac{\partial^{2} h_{U_{1}}}{\partial \varphi_{1}^{\prime} \partial s_{1}}=2 \frac{\partial}{\partial s_{1}}\left[D_{\xi_{1}} \frac{\partial \rho_{\xi_{1}}^{(1)}}{\partial \varphi_{1}^{\prime}}\left(\rho_{\xi_{1}}^{(1)}+\rho_{\xi_{1}}^{(2)}\right)\right]=2 \frac{\partial}{\partial s_{1}}\left[D_{\xi_{1}}\left(\varphi_{1}^{\prime}+v_{1}^{\prime \prime} w_{1}^{\prime}\right)\right] \\
\frac{\partial h_{U_{1}}}{\partial \varphi_{1}}=2\left[D_{\eta_{1}} \frac{\partial \rho_{\eta_{1}}^{(2)}}{\partial \varphi_{1}}\left(\rho_{\eta_{1}}^{(1)}\right)+D_{\zeta_{1}} \frac{\partial \rho_{\zeta_{1}}^{(2)}}{\partial \varphi_{1}}\left(\rho_{\zeta_{1}}^{(1)}\right)\right]=-2 D_{\eta_{1}} v_{1}^{\prime \prime} w_{1}^{\prime \prime}+2 D_{\zeta_{1}} v_{1}^{\prime \prime} w_{1}^{\prime \prime}
\end{gathered}
$$

-Secondary beam,

a) Axial motion equation terms $\left(\delta u_{2}\right)$,

$$
\begin{aligned}
& \frac{\partial^{2} h_{T_{2}}}{\partial \dot{u}_{2} \partial t}=2 m_{2} \frac{\partial}{\partial t}\left[\frac{\partial V_{x_{2}}^{(2)}}{\partial \dot{u}_{2}}\left(V_{x_{2}}^{(1)}+V_{x_{2}}^{(2)}+V_{x_{2}}^{(3 *)}\right)\right]= \\
& =2 m_{2}\left(\ddot{v}_{1 c}+\ddot{u}_{2}+\ddot{v}_{1 c}^{\prime} W_{2}+\dot{v}_{1 c}^{\prime} \dot{W}_{2}+s_{2} \ddot{v}_{1 c}^{\prime} v_{1 c}^{\prime}+s_{2} \dot{v}_{1 C}^{\prime 2}-\right. \\
& \left.-\ddot{\varphi}_{1 c} V_{2}-\dot{\varphi}_{1 c} \dot{V}_{2}+s_{2} \dot{\varphi}_{1 C}^{2}+s_{2} \varphi_{1 c} \ddot{\varphi}_{1 c}+\dot{\varphi}_{1 c} \dot{w}_{1 c}+\varphi_{1 c} \ddot{w}_{1 c}+\ddot{\varphi}_{1 c} w_{1 c}+\dot{\varphi}_{1 c} \dot{w}_{1 c}\right) \text {, } \\
& \frac{\partial h_{T_{2}}}{\partial u_{2}}=2 m_{2}\left[\frac{\partial V_{y_{2}}^{(3 *)}}{\partial u_{2}}\left(V_{y_{2}}^{(1)}+V_{y_{2}}^{(2)}\right)+\frac{\partial V_{z_{2}}^{(3 *)}}{\partial u_{2}}\left(V_{z_{2}}^{(1)}+V_{z_{2}}^{(2)}\right)\right]= \\
& =2 m_{2} \dot{V}_{2} \dot{\varphi}_{1 c}-2 m_{2} \dot{W}_{2} \dot{v}_{1 c}^{\prime} \\
& \frac{\partial^{2} h_{F_{2}}}{\partial u_{2}^{\prime} \partial s_{2}}=-2 \frac{\partial}{\partial s_{2}}\left[\lambda_{2}\left(1+u_{2}^{\prime}\right)\right] \text {, } \\
& \frac{\partial^{3} h_{U_{2}}}{\partial u_{2}^{\prime \prime} \partial s_{2}^{2}}=2 \frac{\partial^{2}}{\partial s_{2}^{2}}\left[D_{\eta_{2}} \frac{\partial \rho_{\eta_{2}}^{(3)}}{\partial u_{2}^{\prime \prime}}\left(\rho_{\eta_{2}}^{(1)}\right)+D_{\zeta_{2}} \frac{\partial \rho_{\zeta_{2}}^{(3)}}{\partial u_{2}^{\prime \prime}}\left(\rho_{\zeta_{2}}^{(1)}\right)\right]= \\
& =-2 \frac{\partial^{2}}{\partial s_{2}^{2}}\left[D_{\eta_{2}}\left(W_{2}^{\prime} W_{2}^{\prime \prime}+v_{1 c}^{\prime} W_{2}^{\prime \prime}\right)\right]+2 \frac{\partial^{2}}{\partial s_{2}^{2}}\left[D_{\zeta_{2}}\left(-V_{2}^{\prime} V_{2}^{\prime \prime}+\varphi_{1 c} V_{2}^{\prime \prime}\right)\right], \\
& \frac{\partial^{2} h_{U_{2}}}{\partial u_{2}^{\prime} \partial s_{2}}=2 \frac{\partial}{\partial s_{2}}\left[D_{\eta_{2}} \frac{\partial \rho_{\eta_{2}}^{(3)}}{\partial W_{2}^{\prime}} \rho_{\eta_{2}}^{(1)}+D_{\zeta_{2}} \frac{\partial \rho_{\zeta_{2}}^{(3)}}{\partial W_{2}^{\prime}} \rho_{\zeta_{2}}^{(1)}\right]=-2 \frac{\partial}{\partial s_{2}}\left[D_{\eta_{2}}\left(W_{2}^{\prime \prime}\right)^{2}\right]-2 \frac{\partial}{\partial s_{2}}\left[D_{\zeta_{2}}\left(V_{2}^{\prime \prime}\right)^{2}\right] \text {, } \\
& \frac{\partial^{2} h_{T_{2}}}{\partial u_{2}^{\prime} \partial s_{2}}=2 \frac{\partial}{\partial s_{2}}\left[I_{\eta_{2}} \frac{\partial \omega_{\eta_{2}}^{(3)}}{\partial \dot{u}_{2}^{\prime}}\left(\omega_{\eta_{2}}^{(1)}\right)+I_{\zeta_{2}} \frac{\partial \omega_{\zeta_{2}}^{(3)}}{\partial u_{2}^{\prime}} \omega_{\zeta_{2}}^{(1)}\right]= \\
& =2 \frac{\partial}{\partial s_{2}}\left[I_{\eta_{2}} \delta_{3}\left(-\dot{W}_{2}^{\prime 2}+\dot{v}_{1 c}^{\prime} \dot{W}_{2}^{\prime}\right)\right]+2 \frac{\partial}{\partial s_{2}}\left[I_{\zeta_{2}} \delta_{2}\left(-\dot{V}_{2}^{\prime 2}+\dot{\varphi}_{1 c} \dot{V}_{2}^{\prime}\right)\right] \text {, } \\
& \frac{\partial^{3} h_{T_{2}}}{\partial \dot{u}_{2}^{\prime} \partial s_{2} \partial t}=2 \frac{\partial^{2}}{\partial s_{2} \partial t}\left[I_{\eta_{2}} \frac{\partial \omega_{\eta_{2}}^{(3 *)}}{\partial \dot{u}_{2}^{\prime}}\left(\omega_{\eta_{2}}^{(1)}\right)+I_{\zeta_{2}} \frac{\partial \omega_{\zeta_{2}}^{(3 *)}}{\partial u_{2}^{\prime}} \omega_{\zeta_{2}}^{(1)}\right]=
\end{aligned}
$$


$=2 \frac{\partial}{\partial s_{2}}\left[I_{\eta_{2}} \delta_{3}\left(-\dot{W}_{2}^{\prime 2}-W_{2}^{\prime} \ddot{W}_{2}^{\prime}+\dot{v}_{1 c} \dot{W}_{2}^{\prime}+v_{1 c} \ddot{W}_{2}^{\prime}\right)\right]+2 \frac{\partial}{\partial s_{2}}\left[I_{\zeta_{2}} \delta_{2}\left(-\dot{V}_{2}^{\prime 2}-\ddot{V}_{2}^{\prime} V_{2}^{\prime}+\dot{\varphi}_{1 c} \dot{V}_{2}^{\prime}+\varphi_{1 c} \ddot{V}_{2}^{\prime}\right)\right]$

b) In-plane bending equation terms $\left(\delta W_{2}\right)$,

$$
\begin{aligned}
& \frac{\partial^{2} h_{T_{2}}}{\partial \dot{W}_{2} \partial t}=2 m_{2} \frac{\partial}{\partial t}\left[\frac{\partial V_{z_{2}}^{(1)}}{\partial \dot{W}_{2}}\left(V_{z_{2}}^{(1)}+V_{z_{2}}^{(2)}\right)\right]= \\
& =2 m_{2}\left(\ddot{w}_{2}+\dot{v}_{1 c}^{\prime} \dot{v}_{1 c}+v_{1 c}^{\prime} \ddot{v}_{1 c}+\dot{w}_{1 c}^{\prime} \dot{w}_{1 c}+w_{1 c}^{\prime} \ddot{w}_{1 c}-\dot{V}_{2} \dot{w}_{1 c}^{\prime}-V_{2} \ddot{w}_{1 c}^{\prime}+\dot{w}_{1 c} \dot{w}_{1 c}^{\prime}+w_{1 c} \ddot{w}_{1 c}^{\prime}\right),(\mathrm{B} .6 \mathrm{a}) \\
& \frac{\partial^{3} h_{T_{2}}}{\partial \dot{W}_{2}^{\prime} \partial s_{2} \partial t}=2 \frac{\partial^{2}}{\partial s_{2} \partial t}\left[I_{\zeta_{2}} \frac{\partial \omega_{\zeta_{2}}^{(2)}}{\partial \dot{W}_{2}^{\prime}}\left(\omega_{\zeta_{2}}^{(1)}\right)+I_{\eta_{2}} \frac{\partial \omega_{\eta_{2}}^{(1)}}{\partial \dot{W}_{2}^{\prime}}\left(\omega_{\eta_{2}}^{(1)}+\omega_{\eta_{2}}^{(2)}\right)\right]= \\
& =2 \frac{\partial}{\partial s_{2}}\left[I_{\zeta_{2}} \delta_{2}\left(\dot{\phi}_{2} \dot{V}_{2}^{\prime}+\phi_{2} \ddot{V}_{2}^{\prime}+\dot{w}_{1 c}^{\prime} \dot{V}_{2}^{\prime}+w_{1 c}^{\prime} \ddot{V}_{2}^{\prime}\right)\right]-2 \frac{\partial}{\partial s_{2}}\left[I _ { \eta _ { 2 } } \delta _ { 3 } \left(-\ddot{W}_{2}^{\prime}+\dot{\phi}_{2} \dot{V}_{2}^{\prime}+\phi_{2} \ddot{V}_{2}^{\prime}+\dot{w}_{1 c}^{\prime} \dot{V}_{2}^{\prime}+\right.\right. \\
& \left.\left.+w_{1 c}^{\prime} \ddot{V}_{2}^{\prime}-\dot{\phi}_{2} \dot{\varphi}_{1 c}-\phi_{2} \ddot{\varphi}_{1 c}-\dot{w}_{1 c}^{\prime} \dot{\varphi}_{1 c}-w_{1 c}^{\prime} \ddot{\varphi}_{1 c}+\ddot{w}_{1 c}^{\prime} \varphi_{1 c}+\dot{w}_{1 c}^{\prime} \dot{\varphi}_{1 c}\right)\right] \text {, } \\
& \frac{\partial h_{T_{2}}}{\partial W_{2}}=2 m_{2}\left[\frac{\partial V_{x_{2}}^{(2)}}{\partial W_{2}}\left(V_{x_{2}}^{(1)}\right)+\frac{\partial V_{y_{2}}^{(2)}}{\partial W_{2}}\left(V_{y_{2}}^{(1)}\right)\right]=2 m_{2} \dot{v}_{1 c}^{\prime} \dot{v}_{1 c}+2 m_{2} \dot{W}_{1 c}^{\prime} \dot{V}_{2} \\
& \frac{\partial^{2} h_{T_{2}}}{\partial W_{2}^{\prime} \partial s_{2}}=2 \frac{\partial}{\partial s_{2}}\left[I_{\xi_{2}} \frac{\partial \omega_{\xi_{2}}^{(2)}}{\partial W_{2}^{\prime}}\left(\omega_{\xi_{2}}^{(1)}+\omega_{\xi_{2}}^{(2)}\right)+I_{\zeta_{2}} \frac{\partial \omega_{\zeta_{2}}^{(3)}}{\partial W_{2}^{\prime}} \omega_{\zeta_{2}}^{(1)}+I_{\eta_{2}} \frac{\partial \omega_{\eta_{2}}^{(3)}}{\partial W_{2}^{\prime}} \omega_{\eta_{2}}^{(1)}\right]= \\
& =2 \frac{\partial}{\partial s_{2}}\left[I_{\xi_{2}}\left(\dot{V}_{2}^{\prime} \dot{\phi}_{2}-\dot{\varphi}_{1 c} \dot{\phi}_{2}\right)\right] \text {, } \\
& \frac{\partial^{2} h_{F_{2}}}{\partial W_{2}^{\prime} \partial s_{2}}=-2 \frac{\partial}{\partial s_{2}}\left[\lambda_{2}\left(W_{2}^{\prime}+v_{1 C}^{\prime}\right)\right] \\
& \frac{\partial^{3} h_{U_{2}}}{\partial W_{2}^{\prime \prime} \partial s_{2}^{2}}=2 \frac{\partial^{2}}{\partial s_{2}^{2}}\left[D_{\eta_{2}} \frac{\partial \rho_{\eta_{2}}^{(1)}}{\partial W_{2}^{\prime \prime}}\left(\rho_{\eta_{2}}^{(1)}+\rho_{\eta_{2}}^{(2)}\right)+D_{\zeta_{2}} \frac{\partial \rho_{\zeta_{2}}^{(2)}}{\partial W_{2}^{\prime \prime}}\left(\rho_{\zeta_{2}}^{(1)}+\rho_{\zeta_{2}}^{(2)}\right)\right]= \\
& =-2 \frac{\partial^{2}}{\partial s_{2}^{2}}\left[D_{\eta_{2}}\left(-W_{2}^{\prime \prime}+\phi_{2} V_{2}^{\prime \prime}+w_{1 C}^{\prime} V_{2}^{\prime \prime}\right)\right]+2 \frac{\partial^{2}}{\partial s_{2}^{2}}\left[D_{\zeta_{2}}\left(V_{2}^{\prime \prime} \phi_{2}+V_{2}^{\prime \prime} w_{1 C}^{\prime}\right)\right] \text {, } \\
& \frac{\partial^{2} h_{U_{2}}}{\partial W_{2}^{\prime} \partial s_{2}}=2 \frac{\partial}{\partial s_{2}}\left[D_{\xi_{2}} \frac{\partial \rho_{\xi_{2}}^{(2)}}{\partial W_{2}^{\prime}}\left(\rho_{\xi_{2}}^{(1)}+\rho_{\xi_{2}}^{(2)}\right)\right]=2 \frac{\partial}{\partial s_{2}}\left[D_{\xi_{2}} \phi_{2}^{\prime} V_{2}^{\prime \prime}\right] \text {. }
\end{aligned}
$$

c) Out-of-plane bending equation terms $\left(\delta V_{2}\right)$,

$$
\begin{gathered}
\frac{\partial^{2} h_{T_{2}}}{\partial \dot{V}_{2} \partial t}=2 m_{2} \frac{\partial}{\partial t}\left[\frac{\partial V_{y_{2}}^{(1)}}{\partial \dot{V}_{2}}\left(V_{y_{2}}^{(1)}+V_{y_{2}}^{(2)}\right)\right]= \\
=2 m_{2}\left(\ddot{V}_{2}-\dot{\varphi}_{1 c} \dot{v}_{1 c}-\varphi_{1 c} \ddot{v}_{1 c}+\dot{W}_{2} \dot{w}_{1 c}^{\prime}+W_{2} \ddot{w}_{1 c}^{\prime}+s_{2} \ddot{v}_{1 c}^{\prime} w_{1 c}^{\prime}+s_{2} \dot{v}_{1 c}^{\prime} \dot{w}_{1 c}^{\prime}+s_{2} \dot{v}_{1 c}^{\prime} \dot{w}_{1 c}^{\prime}+s_{2} v_{1 c}^{\prime} \ddot{w}_{1 c}^{\prime}\right), \\
\frac{\partial^{3} h_{T_{2}}}{\partial \dot{V}_{2}^{\prime} \partial s_{2} \partial t}=2 \frac{\partial^{2}}{\partial s_{2} \partial t}\left[I_{\xi_{2}} \frac{\partial \omega_{\xi_{2}}^{(2)}}{\partial \dot{V}_{2}^{\prime}}\left(\omega_{\xi_{2}}^{(1)}\right)+I_{\zeta_{2}} \frac{\partial \omega_{\zeta_{2}}^{(1)}}{\partial \dot{V}_{2}^{\prime}}\left(\omega_{\zeta_{2}}^{(1)}+\omega_{\zeta_{2}}^{(2)}\right)+I_{\eta_{2}} \frac{\partial \omega_{\eta_{2}}^{(2)}}{\partial \dot{V}_{2}^{\prime}}\left(\omega_{\eta_{2}}^{(1)}\right)\right]= \\
=2 \frac{\partial}{\partial s_{2}}\left[I_{\xi_{2}}\left(\dot{W}_{2}^{\prime} \dot{\phi}_{2}+W_{2}^{\prime} \ddot{\phi}_{2}+\dot{v}_{1 c}^{\prime} \dot{\phi}_{2}+v_{1 c}^{\prime} \ddot{\phi}_{2}\right)\right]+
\end{gathered}
$$




$$
\begin{aligned}
& +2 \frac{\partial}{\partial s_{2}}\left[I_{\zeta_{2}} \delta_{2}\left(\ddot{V}_{2}^{\prime}+\ddot{W}_{2}^{\prime} \phi_{2}+\dot{\phi}_{2} \dot{W}_{2}^{\prime}+\ddot{v}_{1 c}^{\prime} \phi_{2}+\dot{v}_{1 c}^{\prime} \dot{\phi}_{2}+\ddot{W}_{2}^{\prime} w_{1 c}^{\prime}+\dot{W}_{2}^{\prime} \dot{w}_{1 c}^{\prime}+2 \ddot{v}_{1 c}^{\prime} w_{1 c}^{\prime}+2 \dot{v}_{1 c}^{\prime} \dot{w}_{1 c}^{\prime}\right)\right]+ \\
& -2 \frac{\partial}{\partial s_{2}}\left[I_{\eta_{2}} \delta_{3}\left(\dot{\phi}_{2} \dot{W}_{2}^{\prime}+\phi_{2} \ddot{W}_{2}^{\prime}+\dot{w}_{1 c}^{\prime} \dot{W}_{2}^{\prime}+w_{1 c}^{\prime} \ddot{W}_{2}^{\prime}\right)\right], \\
& \frac{\partial h_{T_{2}}}{\partial V_{2}}=2 m_{2}\left[\frac{\partial V_{x_{2}}^{(2)}}{\partial V_{2}}\left(V_{x_{2}}^{(1)}\right)+\frac{\partial V_{z_{2}}^{(2)}}{\partial V_{2}}\left(V_{z_{2}}^{(1)}\right)\right]=-2 m_{2} \dot{\varphi}_{1 c} \dot{v}_{1 c}-2 m_{2} \dot{w}_{1 c}^{\prime} \dot{W}_{2}, \\
& \frac{\partial^{2} h_{F_{2}}}{\partial V_{2}^{\prime} \partial s_{2}}=-2 \frac{\partial}{\partial s_{2}}\left[\lambda_{2}\left(V_{2}^{\prime}-\varphi_{1 C}\right)\right] \\
& \frac{\partial^{3} h_{U_{2}}}{\partial V_{2}^{\prime \prime} \partial s_{2}^{2}}=2 \frac{\partial^{2}}{\partial s_{2}^{2}}\left[D_{\xi_{2}} \frac{\partial \rho_{\xi_{2}}^{(2)}}{\partial V_{2}^{\prime \prime}}\left(\rho_{\xi_{2}}^{(1)}\right)+D_{\eta_{2}} \frac{\partial \rho_{\eta_{2}}^{(2)}}{\partial V_{2}^{\prime \prime}}\left(\rho_{\eta_{2}}^{(1)}\right)+D_{\zeta_{2}} \frac{\partial \rho_{\zeta_{2}}^{(1)}}{\partial V_{2}^{\prime \prime}}\left(\rho_{\zeta_{2}}^{(1)}+\rho_{\zeta_{2}}^{(2)}\right)\right]= \\
& =2 \frac{\partial^{2}}{\partial s_{2}^{2}}\left[D_{\xi_{2}}\left(W_{2}^{\prime} \phi_{2}^{\prime}+v_{1 C}^{\prime} \phi_{2}^{\prime}\right)\right]+2 \frac{\partial^{2}}{\partial s_{2}^{2}}\left[D_{\eta_{2}}\left(-\phi_{2} W_{2}^{\prime \prime}-w_{1 C}^{\prime} W_{2}^{\prime \prime}\right)\right]+ \\
& +2 \frac{\partial^{2}}{\partial s_{2}^{2}}\left[D_{\zeta_{2}}\left(V_{2}^{\prime \prime}+\phi_{2} W_{2}^{\prime \prime}+w_{1 C}^{\prime} W_{2}^{\prime \prime}\right)\right] \\
& \frac{\partial^{2} h_{T_{2}}}{\partial V_{2}^{\prime} \partial s_{2}}=2 \frac{\partial}{\partial s_{2}}\left[I_{\zeta_{2}} \frac{\partial \omega_{\zeta_{2}}^{(3)}}{\partial V_{2}^{\prime}} \omega_{\zeta_{2}}^{(1)}\right]=-2 \frac{\partial}{\partial s_{2}}\left[I_{\zeta_{2}} \dot{V}_{2}^{\prime} \dot{u}_{2}^{\prime}\right], \text { H.O.T. } \\
& \frac{\partial^{2} h_{U_{2}}}{\partial V_{2}^{\prime} \partial s_{2}}=2 \frac{\partial}{\partial s_{2}}\left[D_{\zeta_{2}} \frac{\partial \rho_{\zeta_{2}}^{(3)}}{\partial V_{2}^{\prime}} \rho_{\zeta_{2}}^{(1)}\right]=-2 \frac{\partial}{\partial s_{2}}\left[D_{\zeta_{2}} V_{2}^{\prime \prime} u_{2}^{\prime \prime}\right] \text {, H.O.T. }
\end{aligned}
$$

d) Torsional motion equation terms $\left(\delta \phi_{2}\right)$,

$$
\begin{gathered}
\frac{\partial^{2} h_{T_{2}}}{\partial \dot{\phi}_{2} \partial t}=2 \frac{\partial}{\partial t}\left[I_{\xi_{2}} \frac{\partial \omega_{\xi_{2}}^{(1)}}{\partial \dot{\phi}_{2}}\left(\omega_{\xi_{2}}^{(1)}+\omega_{\xi_{2}}^{(2)}\right)\right]= \\
=2 I_{\xi_{2}}\left(\ddot{\phi}_{2}+\ddot{V}_{2}^{\prime} W_{2}^{\prime}+\dot{V}_{2}^{\prime} \dot{W}_{2}^{\prime}+\ddot{V}_{2}^{\prime} v_{1 C}^{\prime}+\dot{V}_{2}^{\prime} \dot{v}_{1 C}^{\prime}-\ddot{\varphi}_{1 c} W_{2}^{\prime}-\dot{\varphi}_{1 c} \dot{W}_{2}^{\prime}-\ddot{\varphi}_{1 c} v_{1 C}^{\prime}-\dot{\varphi}_{1 c} \dot{v}_{1 C}^{\prime}+\right. \\
\left.+\delta_{1} \dot{\varphi}_{1 c} \dot{v}_{1 c}^{\prime}+\delta_{1} \varphi_{1 c} \ddot{v}_{1 c}^{\prime}\right), \\
\frac{\partial h_{T_{2}}}{\partial \phi_{2}}=2\left[I_{\eta_{2}} \frac{\partial \omega_{\eta_{2}}^{(2)}}{\partial \phi_{2}}\left(\omega_{\eta_{2}}^{(1)}\right)+I_{\zeta_{2}} \frac{\partial \omega_{\zeta_{2}}^{(2)}}{\partial \phi_{2}}\left(\omega_{\zeta_{2}}^{(1)}\right)\right]= \\
=2 I_{\eta_{2}} \delta_{3}\left(-\dot{V}_{2}^{\prime} \dot{W}_{2}^{\prime}+\dot{\varphi}_{1 c} \dot{W}_{2}^{\prime}\right)+2 I_{\zeta_{2}} \delta_{2}\left(\dot{V}_{2}^{\prime} \dot{W}_{2}^{\prime}+\dot{V}_{2}^{\prime} \dot{v}_{1 c}^{\prime}\right), \\
\frac{\partial^{2} h_{U_{2}}}{\partial \phi_{2}^{\prime} \partial s_{2}}=2 \frac{\partial}{\partial s_{2}}\left[D_{\xi_{2}} \frac{\partial \rho_{\xi_{2}}^{(1)}}{\partial \phi_{2}^{\prime}}\left(\rho_{\xi_{2}}^{(1)}+\rho_{\xi_{2}}^{(2)}\right)\right]=2 \frac{\partial}{\partial s_{2}}\left[D_{\xi_{2}}\left(\phi_{2}^{\prime}+V_{2}^{\prime \prime} W_{2}^{\prime}+v_{1 C}^{\prime} V_{2}^{\prime \prime}\right)\right], \\
\frac{\partial h_{U_{2}}}{\partial \phi_{2}}=2\left[D_{\eta_{2}} \frac{\partial \rho_{\eta_{2}}^{(2)}}{\partial \phi_{2}}\left(\rho_{\eta_{2}}^{(1)}\right)+D_{\zeta_{2}} \frac{\partial \rho_{\zeta_{2}}^{(2)}}{\partial \phi_{2}}\left(\rho_{\zeta_{2}}^{(1)}\right)\right]=-2 D_{\eta_{2}} W_{2}^{\prime \prime} V_{2}^{\prime \prime}+2 D_{\zeta_{2}} W_{2}^{\prime \prime} V_{2}^{\prime \prime} .
\end{gathered}
$$

Considering the variations in the strong boundary conditions in equations (43) - (50) then the explicit form of each term in equation (22) for the weak boundary conditions is:

-Primary beam,

a) axial motion $\left(u_{1}\right)$, 


$$
\begin{gathered}
\left.\left(-\frac{1}{2} \frac{\partial^{2} h_{T_{1}}}{\partial \dot{u}_{1}^{\prime} \partial t}+\frac{1}{2} \frac{\partial h_{T_{1}}}{\partial u_{1}^{\prime}}+\frac{1}{2} \frac{\partial^{2} h_{U_{1}}}{\partial u_{1}^{\prime \prime} \partial s_{1}}-\frac{1}{2} \frac{\partial^{2} h_{U_{1}}}{\partial u_{1}^{\prime}}+\frac{1}{2} \frac{\partial h_{F_{1}}}{\partial u_{1}^{\prime}}\right) \delta u_{1}\right|_{s_{1}=0} ^{s_{1}=l_{1}}= \\
=\left.\left[I_{\zeta_{1}} \delta_{3} v_{1}^{\prime} \ddot{v}_{1}^{\prime}\right]\right|_{S_{1}=l_{1}}+\left.\left[I_{\eta_{1}} \delta_{1} w_{1}^{\prime} \ddot{w}_{1}^{\prime}\right]\right|_{s_{1}=l_{1}}-\left.\left[\frac{\partial}{\partial s_{1}}\left(D_{\eta_{1}} w_{1}^{\prime} w_{1}^{\prime \prime}\right)\right]\right|_{s_{1}=l_{1}}-\left.\left[\frac{\partial}{\partial s_{1}}\left(D_{\zeta_{1}} v_{1}^{\prime} v_{1}^{\prime \prime}\right)\right]\right|_{S_{1}=l_{1}}+ \\
+\left.\left[D_{\eta_{1}}{w_{1}^{\prime \prime}}^{\prime 2}\right]\right|_{s_{1}=l_{1}}+\left.\left[D_{\zeta_{1}} v_{1}^{\prime \prime 2}\right]\right|_{s_{1}=l_{1}}-\left.\left[\lambda_{1}\left(1+u_{1}^{\prime}\right)\right]\right|_{s_{1}=l_{1}} \delta u_{1 C} \\
-\int_{0}^{l_{2}}\left(\frac{1}{2} \frac{\partial^{2} h_{T_{2}}}{\partial \dot{u}_{1 C} \partial t}-\frac{1}{2} \frac{\partial h_{T_{2}}}{\partial u_{1 C}}+q_{z, 2}\right) d s_{2} \delta u_{1 C}= \\
=-\int_{0}^{l_{2}}\left(m_{2} \frac{1}{2} \frac{\partial}{\partial t}\left[\frac{\partial V_{x_{2}}^{(3)}}{\partial \dot{u}_{1 C}}\left(V_{x_{2}}^{(1)}\right)\right]+m_{2} \frac{1}{2} \frac{\partial}{\partial t}\left[\frac{\partial V_{y_{2}}^{(3)}}{\partial \dot{u}_{1 C}}\left(V_{y_{2}}^{(1)}\right)\right]-\right. \\
\left.-m_{2} \frac{1}{2}\left[\frac{\partial V_{x_{2}}^{(3)}}{\partial u_{1 C}}\left(V_{x_{2}}^{(1)}\right)\right]-m_{2} \frac{1}{2}\left[\frac{\partial V_{y_{2}}^{(3)}}{\partial u_{1 C}}\left(V_{y_{2}}^{(1)}\right)\right]+q_{z, 2}\right) d s_{2} \delta u_{1 C}= \\
=-\int_{0}^{l_{2}}\left\{m_{2}\left(-\dot{v}_{1 c}^{\prime} \dot{v}_{1 c}-v_{1 c}^{\prime} \ddot{v}_{1 c}\right)-m_{2}\left(\dot{w}_{1 c}^{\prime} \dot{V}_{2}+w_{1 c}^{\prime} \ddot{V}_{2}\right)+\right. \\
\left.+m_{2} \dot{v}_{1 c}^{\prime} \dot{v}_{1 c}+m_{2} \dot{V}_{2} \dot{w}_{1 c}^{\prime}+q_{z, 2}\right\} d s_{2} \delta u_{1 C} .
\end{gathered}
$$

By taking equation (49a) it can be found from equation (22) that,

$$
\begin{gathered}
-\left.\left(-\frac{1}{2} \frac{\partial^{2} h_{T_{2}}}{\partial \dot{W}_{2}^{\prime} \partial t}+\frac{1}{2} \frac{\partial h_{T_{2}}}{\partial W_{2}^{\prime}}+\frac{1}{2} \frac{\partial h_{F_{2}}}{\partial W_{2}^{\prime}}+\frac{1}{2} \frac{\partial^{2} h_{U_{2}}}{\partial W_{2}^{\prime \prime} \partial s_{2}}-\frac{1}{2} \frac{\partial h_{U_{2}}}{\partial W_{2}^{\prime}}-M_{z, 2}\right)\right|_{S_{2}=0} \delta u_{1 C}= \\
=-\left\{-\frac{1}{2} \frac{\partial}{\partial t}\left[I_{\zeta_{2}} \frac{\partial \omega_{\zeta_{2}}^{(2)}}{\partial \dot{W}_{2}^{\prime}}\left(\omega_{\zeta_{2}}^{(1)}\right)+I_{\eta_{2}} \frac{\partial \omega_{\eta_{2}}^{(1)}}{\partial \dot{W}_{2}^{\prime}}\left(\omega_{\eta_{2}}^{(1)}+\omega_{\eta_{2}}^{(2)}\right)\right]+\frac{1}{2}\left[I_{\xi_{2}} \frac{\partial \omega_{\xi_{2}}^{(2)}}{\partial W_{2}^{\prime}}\left(\omega_{\xi_{2}}^{(1)}\right)\right]-\right. \\
-\lambda_{2}\left(W_{2}^{\prime}+v_{1 C}^{\prime}\right)+\frac{1}{2} \frac{\partial}{\partial s_{2}}\left[D_{\eta_{2}} \frac{\partial \rho_{\eta_{2}}^{(1)}}{\partial W_{2}^{\prime \prime}}\left(\rho_{\eta_{2}}^{(1)}+\rho_{\eta_{2}}^{(2)}\right)+\right. \\
=\left\{\left.\left[I_{\zeta_{2}} \delta_{2}\left(\phi_{2} \ddot{V}_{2}^{\prime}+\dot{w}_{1 c}^{\prime} \dot{V}_{2}^{\prime}+\phi_{2} \dot{V}_{2}^{\prime}+w_{1 c}^{\prime} \ddot{V}_{2}^{\prime}\right)\right]\right|_{S_{2}=0}-\left[I _ { \eta _ { 2 } } \delta _ { 3 } \left(-\ddot{W}_{2}^{\prime}+\dot{\phi}_{2} \dot{V}_{2}^{\prime}+\phi_{2} \ddot{V}_{2}^{\prime}+\dot{w}_{1 c}^{\prime} \dot{V}_{2}^{\prime}+\right.\right.\right. \\
\left.\left.\left.+D_{\zeta_{2}} \frac{\partial \rho_{\zeta_{2}}^{(2)}}{\partial W_{2}^{\prime \prime}}\left(\rho_{\zeta_{2}}^{(1)}\right)\right]-\frac{1}{2}\left[D_{\xi_{2}} \frac{\partial \rho_{\xi_{2}}^{(2)}}{\partial W_{2}^{\prime}}\left(\rho_{\xi_{2}}^{(1)}\right)\right]-M_{z, 2} \dot{\varphi}_{1 c}-\phi_{2} \ddot{\varphi}_{1 c}-\dot{w}_{1 c}^{\prime} \dot{\varphi}_{1 c}-w_{1 c}^{\prime} \ddot{\varphi}_{1 c}+\ddot{w}_{1 c}^{\prime} \varphi_{1 c}+\dot{w}_{1 c}^{\prime} \dot{\varphi}_{1 c}\right)\right]\left.\right|_{S_{2}=0}- \\
-\left.\left[I_{\xi_{2}}\left(\dot{V}_{2}^{\prime} \dot{\phi}_{2}-\varphi_{1 c} \dot{\phi}_{2}\right)\right]\right|_{S_{2}=0}+\left.\left[\lambda_{2}\left(W_{2}^{\prime}+v_{1 C}^{\prime}\right)\right]\right|_{s_{2}=0}+\left.\left\{\frac{\partial}{\partial s_{2}}\left[D_{\eta_{2}}\left(-W_{2}^{\prime \prime}+\phi_{2} V_{2}^{\prime \prime} w_{1 C}^{\prime} V_{2}^{\prime \prime}\right)\right]\right\}\right|_{S_{2}=0}- \\
\left.-\left.\left\{\frac{\partial}{\partial s_{2}}\left[D_{\zeta_{2}}\left(V_{2}^{\prime \prime} \phi_{2}+V_{2}^{\prime \prime} w_{1 C}^{\prime}\right)\right]\right\}\right|_{S_{2}=0}+\left.\left[D_{\xi_{2}} \phi_{2}^{\prime} V_{2}^{\prime \prime}\right]\right|_{s_{2}=0}+M_{z, 2}(0, t)\right\} \delta u_{1 C} .
\end{gathered}
$$

b) In-plane bending $\left(v_{1}\right)$,

$$
\left.\left(-\frac{1}{2} \frac{\partial^{2} h_{T_{1}}}{\partial \dot{v}_{1}^{\prime} \partial t}+\frac{1}{2} \frac{\partial h_{T_{1}}}{\partial v_{1}^{\prime}}+\frac{1}{2} \frac{\partial h_{F_{1}}}{\partial v_{1}^{\prime}}+\frac{1}{2} \frac{\partial^{2} h_{U_{1}}}{\partial v_{1}^{\prime \prime} \partial s_{1}}-\frac{1}{2} \frac{\partial h_{U_{1}}}{\partial v_{1}^{\prime}}+M_{y, 1}\right)\right|_{s_{1}=l_{1}} \delta v_{1 C}=
$$




$$
\begin{aligned}
= & \left\{-\frac{1}{2} \frac{\partial}{\partial t}\left[I_{\xi_{1}} \frac{\partial \omega_{\xi_{1}}^{(2)}}{\partial \dot{v}_{1}^{\prime}}\left(\omega_{\xi_{1}}^{(1)}\right)+I_{\eta_{1}} \frac{\partial \omega_{\eta_{1}}^{(2)}}{\partial \dot{v}_{1}^{\prime}}\left(\omega_{\eta_{1}}^{(1)}\right)+I_{\zeta_{1}} \frac{\partial \omega_{\zeta_{1}}^{(1)}}{\partial \dot{v}_{1}^{\prime}}\left(\omega_{\zeta_{1}}^{(1)}+\omega_{\zeta_{1}}^{(2)}\right)\right]+\right. \\
+ & 2 \frac{\partial}{\partial s_{1}}\left[I_{\zeta_{1}} \frac{\partial \omega_{\zeta_{1}}^{(3)}}{\partial v_{1}^{\prime}} \omega_{\zeta_{1}}^{(1)}\right]-\lambda_{1} v_{1}^{\prime}+\frac{1}{2} \frac{\partial}{\partial s_{1}}\left[D_{\xi_{1}} \frac{\partial \rho_{\xi_{1}}^{(2)}}{\partial v_{1}^{\prime \prime}}\left(\rho_{\xi_{1}}^{(1)}\right)+D_{\eta_{1}} \frac{\partial \rho_{\eta_{1}}^{(2)}}{\partial v_{1}^{\prime \prime}}\left(\rho_{\eta_{1}}^{(1)}\right)+\right. \\
& \left.\left.+D_{\zeta_{1}} \frac{\partial \rho_{\zeta_{1}}^{(1)}}{\partial v_{1}^{\prime \prime}}\left(\rho_{\zeta_{1}}^{(1)}+\rho_{\zeta_{1}}^{(2)}\right)\right]-2 \frac{\partial}{\partial s_{1}}\left[D_{\zeta_{1}} \frac{\partial \rho_{\zeta_{1}}^{(3)}}{\partial v_{1}^{\prime}} \rho_{\zeta_{1}}^{(1)}\right]+M_{y, 1}\right\}\left.\right|_{S_{1}=l_{1}} \delta v_{1 C}= \\
=\{ & -I_{\xi_{1}}\left(\dot{w}_{1}^{\prime} \dot{\varphi}_{1}+w_{1}^{\prime} \ddot{\varphi}_{1}\right)+I_{\eta_{1}} \delta_{1}\left(\dot{\varphi}_{1} \dot{w}_{1}^{\prime}+\varphi_{1} \ddot{w}_{1}^{\prime}\right)-I_{\zeta_{1}} \delta_{3}\left(\ddot{v}_{1}^{\prime}+\ddot{w}_{1}^{\prime} \varphi_{1}+\dot{\varphi}_{1} \dot{w}_{1}^{\prime}\right)- \\
-\lambda_{1} v_{1}^{\prime}+ & \left.\frac{\partial}{\partial s_{1}}\left[D_{\xi_{1}} w_{1}^{\prime} \varphi_{1}^{\prime}\right]-\frac{\partial}{\partial s_{1}}\left[D_{\eta_{1}} \varphi_{1} w_{1}^{\prime \prime}\right]+\frac{\partial}{\partial s_{1}}\left[D_{\zeta_{1}}\left(v_{1}^{\prime \prime}+\varphi_{1} w_{1}^{\prime \prime}\right)\right]+M_{y, 1}+H . O . T .\right\}\left.\right|_{s_{1}=l_{1}} \delta v_{1 C},
\end{aligned}
$$

$$
\begin{aligned}
& -\int_{0}^{l_{2}}\left(\frac{1}{2} \frac{\partial^{2} h_{T_{2}}}{\partial \dot{v}_{1 C} \partial t}\right) d s_{2} \delta v_{1 C}=-\frac{1}{2} \int_{0}^{l_{2}} m_{2}\left\{\frac{\partial}{\partial t}\left[\frac{\partial V_{x_{2}}^{(1)}}{\partial \dot{v}_{1 C}}\left(V_{x_{2}}^{(1)}+V_{x_{2}}^{(2)}\right)\right]+\right. \\
& \left.+\frac{\partial}{\partial t}\left[\frac{\partial V_{y_{2}}^{(2)}}{\partial \dot{v}_{1 C}}\left(V_{y_{2}}^{(1)}\right)\right]+\frac{\partial}{\partial t}\left[\frac{\partial V_{z_{2}}^{(2)}}{\partial \dot{v}_{1 C}}\left(V_{z_{2}}^{(1)}\right)\right]\right\} d s_{2} \delta v_{1 C}= \\
& =-\int_{0}^{l_{2}}\left\{m _ { 2 } \left[\ddot{v}_{1 c}+\ddot{u}_{2}+\ddot{v}_{1 c}^{\prime} W_{2}+\dot{v}_{1 c}^{\prime} \dot{W}_{2}+s_{2} \ddot{v}_{1 c}^{\prime} v_{1 c}^{\prime}+s_{2} \dot{v}_{1 c}^{\prime} \dot{v}_{1 c}^{\prime}-\ddot{\varphi}_{1 c} V_{2}-\dot{\varphi}_{1 c} \dot{V}_{2}+s_{2} \dot{\varphi}_{1 c} \dot{\varphi}_{1 c}+\right.\right. \\
& +s_{2} \varphi_{1 c} \ddot{\varphi}_{1 c}+\dot{\varphi}_{1 c} \dot{w}_{1 c}+\varphi_{1 c} \ddot{w}_{1 c}+\ddot{\varphi}_{1 c} w_{1 c}+\dot{\varphi}_{1 c} \dot{w}_{1 c}- \\
& \left.\left.-\ddot{V}_{2} \varphi_{1 c}-\dot{V}_{2} \dot{\varphi}_{1 c}+\dot{v}_{1 c}^{\prime} \dot{W}_{2}+v_{1 c}^{\prime} \ddot{W}_{2}\right]\right\} d s_{2} \delta v_{1 C}, \\
& -\left.\frac{1}{2} \frac{\partial h_{U_{1}}}{\partial v_{1}^{\prime \prime}}\right|_{S_{1}=l_{1}} \delta v_{1 C}^{\prime}=-\left\{\frac { 1 } { 2 } \left[D_{\xi_{1}} \frac{\partial \rho_{\xi_{1}}^{(2)}}{\partial v_{1}^{\prime \prime}}\left(\rho_{\xi_{1}}^{(1)}\right)+D_{\eta_{1}} \frac{\partial \rho_{\eta_{1}}^{(2)}}{\partial v_{1}^{\prime \prime}}\left(\rho_{\eta_{1}}^{(1)}\right)+\right.\right. \\
& \left.\left.+D_{\zeta_{1}} \frac{\partial \rho_{\zeta_{1}}^{(1)}}{\partial v_{1}^{\prime \prime}}\left(\rho_{\zeta_{1}}^{(1)}+\rho_{\zeta_{1}}^{(2)}\right)\right]\right\}_{S_{1}=l_{1}} \delta v_{1 C}^{\prime}= \\
& =\left\{-\left.\left[D_{\xi_{1}} w_{1}^{\prime} \varphi_{1}^{\prime}\right]\right|_{s_{1}=l_{1}}+\left.\left[D_{\eta_{1}} \varphi_{1} w_{1}^{\prime \prime}\right]\right|_{s_{1}=l_{1}}-\left.\left[D_{\zeta_{1}}\left(v_{1}^{\prime \prime}+\varphi_{1} w_{1}^{\prime \prime}\right)\right]\right|_{s_{1}=l_{1}}\right\} \delta v_{1 C}^{\prime} \\
& -\int_{0}^{l_{2}}\left(\frac{1}{2} \frac{\partial^{2} h_{T_{2}}}{\partial \dot{v}_{1 C}^{\prime} \partial t}-\frac{1}{2} \frac{\partial h_{T_{2}}}{\partial v_{1 C}^{\prime}}-\frac{1}{2} \frac{\partial h_{F_{2}}}{\partial v_{1 C}^{\prime}}+\frac{1}{2} \frac{\partial h_{U_{2}}}{\partial v_{1 C}^{\prime}}-s_{2} q_{z, 2}+M_{z, 2}\right) d s_{2} \delta v_{1 C}^{\prime}= \\
& =-\int_{0}^{l_{2}}\left\{m_{2} \frac{1}{2} \frac{\partial}{\partial t}\left[\frac{\partial V_{x_{2}}^{(2)}}{\partial \dot{v}_{1 C}^{\prime}}\left(V_{x_{2}}^{(1)}\right)\right]+m_{2} \frac{1}{2} \frac{\partial}{\partial t}\left[\frac{\partial V_{y_{2}}^{(2)}}{\partial \dot{v}_{1 C}^{\prime}}\left(V_{y_{2}}^{(1)}\right)\right]+I_{\xi_{2}} \frac{1}{2} \frac{\partial}{\partial t}\left[\frac{\partial \omega_{\xi_{2}}^{(2)}}{\partial \dot{v}_{1 C}^{\prime}}\left(\omega_{\xi_{2}}^{(1)}\right)\right]+\right. \\
& +I_{\zeta_{2}} \frac{1}{2} \frac{\partial}{\partial t}\left[\frac{\partial \omega_{\zeta_{2}}^{(2)}}{\partial \dot{v}_{1 C}^{\prime}}\left(\omega_{\zeta_{2}}^{(1)}\right)\right]-m_{2} \frac{1}{2}\left[\frac{\partial V_{x_{2}}^{(2)}}{\partial v_{1 C}^{\prime}}\left(V_{x_{2}}^{(1)}\right)\right]-m_{2} \frac{1}{2}\left[\frac{\partial V_{y_{2}}^{(2)}}{\partial v_{1 C}^{\prime}}\left(V_{y_{2}}^{(1)}\right)\right]-
\end{aligned}
$$




$$
\begin{gathered}
-m_{2} \frac{1}{2}\left[\frac{\partial V_{z_{2}}^{(2)}}{\partial v_{1 C}^{\prime}}\left(V_{z_{2}}^{(1)}\right)\right]-I_{\xi_{2}} \frac{1}{2}\left[\frac{\partial \omega_{\xi_{2}}^{(2)}}{\partial v_{1 C}^{\prime}}\left(\omega_{\xi_{2}}^{(1)}\right)\right]+\lambda_{2}\left(W_{2}^{\prime}+v_{1 C}^{\prime}\right)+ \\
\left.+\frac{1}{2} D_{\xi_{2}} \frac{\partial \rho_{\xi_{2}}^{(2)}}{\partial v_{1 C}^{\prime}}\left(\rho_{\xi_{2}}^{(1)}\right)-s_{2} q_{z, 2}+M_{z, 2}\right\} d s_{2} \delta v_{1 C}^{\prime}= \\
=-\int_{0}^{l_{2}}\left\{m_{2}\left(\dot{W}_{2} \dot{v}_{1 c}+W_{2} \ddot{v}_{1 c}+s_{2} \dot{v}_{1 c}^{\prime} \dot{v}_{1 c}+s_{2} v_{1 c}^{\prime} \ddot{v}_{1 c}\right)+m_{2}\left(\ddot{V}_{2} s_{2} w_{1 c}^{\prime}+\dot{V}_{2} s_{2} \dot{w}_{1 c}^{\prime}\right)+\right. \\
+\delta_{1} I_{\xi_{2}}\left(\dot{\varphi}_{1 c} \dot{\phi}_{2}+\varphi_{1 c} \ddot{\phi}_{2}\right)+\delta_{2} I_{\zeta_{2}}\left(\ddot{V}_{2}^{\prime} \phi_{2}+\dot{V}_{2}^{\prime} \dot{\phi}_{2}+2 \dot{w}_{1 c}^{\prime} \dot{V}_{2}^{\prime}+2 w_{1 c}^{\prime} \ddot{V}_{2}^{\prime}\right)-m_{2} s_{2} \dot{v}_{1 c}^{\prime} \dot{v}_{1 c}- \\
-m_{2} s_{2} \dot{w}_{1 c}^{\prime} \dot{V}_{2}-m_{2} \dot{v}_{1 c} \dot{W}_{2}-I_{\xi_{2}}\left(\dot{\phi}_{2} \dot{V}_{2}^{\prime}-\dot{\varphi}_{1 c} \dot{\phi}_{2}\right)+\lambda_{2}\left(W_{2}^{\prime}+v_{1 C}^{\prime}\right)+ \\
\left.+D_{\xi_{2}} V_{2}^{\prime \prime} \phi_{2}^{\prime}-s_{2} q_{z, 2}+M_{z, 2}\right\} d s_{2} \delta v_{1 C}^{\prime} .
\end{gathered}
$$

Then applying equation (49b) with equation (22) leads to,

$$
\begin{gathered}
-\left.\frac{1}{2} \frac{\partial h_{U_{2}}}{\partial W_{2}^{\prime \prime}}\right|_{S_{2}=0} \delta v_{1 C}^{\prime}=-\left.\frac{1}{2}\left[D_{\eta_{2}} \frac{\partial \rho_{\eta_{2}}^{(1)}}{\partial W_{2}^{\prime \prime}}\left(\rho_{\eta_{2}}^{(1)}+\rho_{\eta_{2}}^{(2)}\right)+D_{\zeta_{2}} \frac{\partial \rho_{\zeta_{2}}^{(2)}}{\partial W_{2}^{\prime \prime}}\left(\rho_{\zeta_{2}}^{(1)}\right)\right]\right|_{S_{2}=0} \delta v_{1 C}^{\prime}= \\
=\left.\left[D_{\eta_{2}}\left(-W_{2}^{\prime \prime}+\phi_{2} V_{2}^{\prime \prime}+w_{1 C}^{\prime} V_{2}^{\prime \prime}\right)-D_{\zeta_{2}}\left(V_{2}^{\prime \prime} \phi_{2}+V_{2}^{\prime \prime} w_{1 C}^{\prime}\right)\right]\right|_{S_{2}=0} \delta v_{1 C}^{\prime} .
\end{gathered}
$$

c) Out-of-plane bending $\left(w_{1}\right)$,

$$
\begin{aligned}
& \left.\left(-\frac{1}{2} \frac{\partial^{2} h_{T_{1}}}{\partial \dot{w}_{1}^{\prime} \partial t}+\frac{1}{2} \frac{\partial h_{T_{1}}}{\partial w_{1}^{\prime}}+\frac{1}{2} \frac{\partial h_{F_{1}}}{\partial w_{1}^{\prime}}+\frac{1}{2} \frac{\partial^{2} h_{U_{1}}}{\partial w_{1}^{\prime \prime} \partial s_{1}}-\frac{1}{2} \frac{\partial h_{U_{1}}}{\partial w_{1}^{\prime}}-M_{z, 1}\right)\right|_{s_{1}=l_{1}} \delta w_{1 C}= \\
& =\left\{-\frac{1}{2} \frac{\partial}{\partial t}\left[I_{\eta_{1}} \frac{\partial \omega_{\eta_{1}}^{(1)}}{\partial \dot{w}_{1}^{\prime}}\left(\omega_{\eta_{1}}^{(1)}+\omega_{\eta_{1}}^{(2)}\right)+I_{\zeta_{1}} \frac{\partial \omega_{\zeta_{1}}^{(2)}}{\partial \dot{w}_{1}^{\prime}}\left(\omega_{\zeta_{1}}^{(1)}\right)\right]+\right. \\
& +\frac{1}{2}\left[I_{\xi_{1}} \frac{\partial \omega_{\xi_{1}}^{(2)}}{\partial w_{1}^{\prime}}\left(\omega_{\xi_{1}}^{(1)}\right)\right]-\lambda_{1} w_{1}^{\prime}+\frac{1}{2} \frac{\partial}{\partial s_{1}}\left[D_{\eta_{1}} \frac{\partial \rho_{\eta_{1}}^{(1)}}{\partial w_{1}^{\prime \prime}}\left(\rho_{\eta_{1}}^{(1)}+\rho_{\eta_{1}}^{(2)}\right)+\right. \\
& \left.\left.+D_{\zeta_{1}} \frac{\partial \rho_{\zeta_{1}}^{(2)}}{\partial w_{1}^{\prime \prime}}\left(\rho_{\zeta_{1}}^{(1)}\right)\right]-\frac{1}{2}\left[D_{\xi_{1}} \frac{\partial \rho_{\xi_{1}}^{(2)}}{\partial w_{1}^{\prime}}\left(\rho_{\xi_{1}}^{(1)}\right)\right]-M_{z, 1}\right\}\left.\right|_{S_{1}=l_{1}} \delta w_{1 C}= \\
& =\left\{\left.\left[I_{\eta_{1}} \delta_{1}\left(-\ddot{w}_{1}^{\prime}+\dot{\varphi}_{1} \dot{v}_{1}^{\prime}+\varphi_{1} \ddot{v}_{1}^{\prime}\right)\right]\right|_{s_{1}=l_{1}}-\left.\left[I_{\zeta_{1}} \delta_{3}\left(\dot{\varphi}_{1} \dot{v}_{1}^{\prime}+\varphi_{1} \ddot{v}_{1}^{\prime}\right)\right]\right|_{s_{1}=l_{1}}+\left.\left[I_{\xi_{1}} \dot{v}_{1}^{\prime} \dot{\varphi}_{1}\right]\right|_{s_{1}=l_{1}}-\right. \\
& -\left.\left[\lambda_{1} w_{1}^{\prime}\right]\right|_{s_{1}=l_{1}}-\left.\left\{\frac{\partial}{\partial s_{1}}\left[D_{\eta_{1}}\left(-w_{1}^{\prime \prime}+\varphi_{1} v_{1}^{\prime \prime}\right)\right]\right\}\right|_{s_{1}=l_{1}}+ \\
& \left.+\left.\left[\frac{\partial}{\partial s_{1}}\left(D_{\zeta_{1}} \varphi_{1} v_{1}^{\prime \prime}\right)\right]\right|_{S_{1}=l_{1}}-\left.\left[D_{\xi_{1}} v_{1}^{\prime \prime} \varphi_{1}^{\prime}\right]\right|_{s_{1}=l_{1}}-M_{z, 1}\left(l_{1}, t\right)\right\} \delta w_{1 C}, \\
& -\int_{0}^{l_{2}}\left(\frac{1}{2} \frac{\partial^{2} h_{T_{2}}}{\partial \dot{w}_{1 C} \partial t}-\frac{1}{2} \frac{\partial h_{T_{2}}}{\partial w_{1 C}}+q_{y, 2}\right) d s_{2} \delta w_{1 C}=
\end{aligned}
$$




$$
\begin{gathered}
=-\int_{0}^{l_{2}}\left\{m_{2} \frac{1}{2} \frac{\partial}{\partial t}\left[\frac{\partial V_{x_{2}}^{(2)}}{\partial \dot{w}_{1 C}}\left(V_{x_{2}}^{(1)}\right)\right]+m_{2} \frac{1}{2} \frac{\partial}{\partial t}\left[\frac{\partial V_{z_{2}}^{(2)}}{\partial \dot{w}_{1 C}}\left(V_{z_{2}}^{(1)}\right)\right]-m_{2} \frac{1}{2}\left[\frac{\partial V_{x_{2}}^{(2)}}{\partial w_{1 C}}\left(V_{x_{2}}^{(1)}\right)\right]-\right. \\
\left.-m_{2} \frac{1}{2}\left[\frac{\partial V_{z_{2}}^{(2)}}{\partial w_{1 C}}\left(V_{z_{2}}^{(1)}\right)\right]+q_{y, 2}\right\} d s_{2} \delta w_{1 C}=-\int_{0}^{l_{2}}\left\{m_{2}\left(\dot{\varphi}_{1 c} \dot{v}_{1 c}+\varphi_{1 c} \ddot{v}_{1 c}\right)+\right. \\
\left.+m_{2}\left(\dot{w}_{1 c}^{\prime} \dot{W}_{2}+w_{1 c}^{\prime} \ddot{W}_{2}\right)-m_{2} \dot{\varphi}_{1 c} \dot{v}_{1 c}-m_{2} \dot{w}_{1 c}^{\prime} \dot{W}_{2}+q_{y, 2}\right\} d s_{2} \delta w_{1 C} .
\end{gathered}
$$

Now, by using equation (50a) and equation (22) one gets,

$$
\begin{aligned}
& -\left.\left(-\frac{1}{2} \frac{\partial^{2} h_{T_{2}}}{\partial \dot{V}_{2}^{\prime} \partial t}+\frac{1}{2} \frac{\partial h_{T_{2}}}{\partial V_{2}^{\prime}}+\frac{1}{2} \frac{\partial h_{F_{2}}}{\partial V_{2}^{\prime}}+\frac{1}{2} \frac{\partial^{2} h_{U_{2}}}{\partial V_{2}^{\prime \prime} \partial s_{2}}+M_{y, 2}\right)\right|_{S_{2}=0} \delta w_{1 C}= \\
& =-\left\{\frac { 1 } { 2 } \frac { \partial } { \partial t } \left[-I_{\xi_{2}} \frac{\partial \omega_{\xi_{2}}^{(2)}}{\partial \dot{V}_{2}^{\prime}}\left(\omega_{\xi_{2}}^{(1)}+\omega_{\xi_{2}}^{(2)}\right)-I_{\zeta_{2}} \frac{\partial \omega_{\zeta_{2}}^{(1)}}{\partial \dot{V}_{2}^{\prime}}\left(\omega_{\zeta_{2}}^{(1)}+\omega_{\zeta_{2}}^{(2)}\right)-\right.\right. \\
& \left.-I_{\eta_{2}} \frac{\partial \omega_{\eta_{2}}^{(2)}}{\partial \dot{V}_{2}^{\prime}}\left(\omega_{\eta_{2}}^{(1)}+\omega_{\eta_{2}}^{(2)}\right)\right]-\left[\lambda_{2}\left(V_{2}^{\prime}-\varphi_{1 C}\right)\right]^{\prime}+ \\
& +\frac{1}{2} \frac{\partial}{\partial s_{2}}\left[D_{\xi_{2}} \frac{\partial \rho_{\xi_{2}}^{(2)}}{\partial V_{2}^{\prime \prime}}\left(\rho_{\xi_{2}}^{(1)}+\rho_{\xi_{2}}^{(2)}\right)+D_{\eta_{2}} \frac{\partial \rho_{\eta_{2}}^{(2)}}{\partial V_{2}^{\prime \prime}}\left(\rho_{\eta_{2}}^{(1)}+\rho_{\eta_{2}}^{(2)}\right)+D_{\zeta_{2}} \frac{\partial \rho_{\zeta_{2}}^{(1)}}{\partial V_{2}^{\prime \prime}}\left(\rho_{\zeta_{2}}^{(1)}+\rho_{\zeta_{2}}^{(2)}\right)\right]+ \\
& \left.+M_{y, 2}\right\}\left.\right|_{S_{2}=0} \delta w_{1 C}=-\left\{-\left.\left[I_{\xi_{2}}\left(\dot{W}_{2}^{\prime} \dot{\phi}_{2}+W_{2}^{\prime} \ddot{\phi}_{2}+\dot{v}_{1 c}^{\prime} \dot{\phi}_{2}+v_{1 c}^{\prime} \ddot{\phi}_{2}\right)\right]\right|_{S_{2}=0}-\left[I _ { \zeta _ { 2 } } \delta _ { 2 } \left(\ddot{V}_{2}^{\prime}+\ddot{W}_{2}^{\prime} \phi_{2}+\right.\right.\right. \\
& \left.\left.+\dot{\phi}_{2} \dot{W}_{2}^{\prime}+\ddot{v}_{1 c}^{\prime} \phi_{2}+\dot{v}_{1 c}^{\prime} \dot{\phi}_{2}+\ddot{W}_{2}^{\prime} w_{1 c}^{\prime}+\dot{W}_{2}^{\prime} \dot{w}_{1 c}^{\prime}+2 \ddot{v}_{1 c}^{\prime} w_{1 c}^{\prime}+2 \dot{v}_{1 c}^{\prime} \dot{w}_{1 c}^{\prime}\right)\right]\left.\right|_{s_{2}=0}+ \\
& +\left.\left[I_{\eta_{2}} \delta_{3}\left(\dot{\phi}_{2} \dot{W}_{2}^{\prime}+\phi_{2} \ddot{W}_{2}^{\prime}+\dot{W}_{1 c}^{\prime} \dot{W}_{2}^{\prime}+w_{1 c}^{\prime} \ddot{W}_{2}^{\prime}\right)\right]\right|_{S_{2}=0}-\left.\left[\lambda_{2}\left(V_{2}^{\prime}-\varphi_{1 C}\right)\right]\right|_{s_{2}=0}+ \\
& +\left.\left\{\frac{\partial}{\partial s_{2}}\left[D_{\xi_{2}}\left(W_{2}^{\prime} \phi_{2}^{\prime}+v_{1 C}^{\prime} \phi_{2}^{\prime}\right)\right]\right\}\right|_{s_{2}=0}-\left.\left\{\frac{\partial}{\partial s_{2}}\left[D_{\eta_{2}}\left(\phi_{2} W_{2}^{\prime \prime}+w_{1 C}^{\prime} W_{2}^{\prime \prime}\right)\right]\right\}\right|_{s_{2}=0}+ \\
& \left.+\left.\left\{\frac{\partial}{\partial s_{2}}\left[D_{\zeta_{2}}\left(V_{2}^{\prime \prime}+\phi_{2} W_{2}^{\prime \prime}+w_{1 C}^{\prime} W_{2}^{\prime \prime}\right)\right]\right\}\right|_{S_{2}=0}+M_{y, 2}(0, t)\right\} \delta w_{1 C}, \\
& -\left.\frac{1}{2} \frac{\partial h_{U_{1}}}{\partial w_{1}^{\prime \prime}}\right|_{s_{1}=l_{1}} \delta w_{1 C}^{\prime}=-\left.\left[\frac{1}{2} D_{\eta_{1}} \frac{\partial \rho_{\eta_{1}}^{(1)}}{\partial w_{1}^{\prime \prime}}\left(\rho_{\eta_{1}}^{(1)}+\rho_{\eta_{1}}^{(2)}\right)+\frac{1}{2} D_{\zeta_{1}} \frac{\partial \rho_{\zeta_{1}}^{(2)}}{\partial w_{1}^{\prime \prime}}\left(\rho_{\zeta_{1}}^{(1)}\right)\right]\right|_{S_{1}=l_{1}} \delta w_{1 C}^{\prime}= \\
& =\left\{\left.\left[D_{\eta_{1}}\left(-w_{1}^{\prime \prime}+\varphi_{1} v_{1}^{\prime \prime}\right)\right]\right|_{s_{1}=l_{1}}-\left.\left[D_{\zeta_{1}} \varphi_{1} v_{1}^{\prime \prime}\right]\right|_{s_{1}=l_{1}}\right\} \delta w_{1 C}^{\prime}, \\
& -\int_{0}^{l_{2}}\left(\frac{1}{2} \frac{\partial^{2} h_{T_{2}}}{\partial \dot{w}_{1 C}^{\prime} \partial t}-\frac{1}{2} \frac{\partial h_{T_{2}}}{\partial w_{1 C}^{\prime}}+\frac{1}{2} \frac{\partial h_{U_{2}}}{\partial w_{1 C}^{\prime}}-M_{x, 2}\right) d s_{2} \delta w_{1 C}^{\prime}= \\
& =-\int_{0}^{l_{2}}\left\{m_{2} \frac{1}{2} \frac{\partial}{\partial t}\left[\frac{\partial V_{y_{2}}^{(2)}}{\partial \dot{w}_{1 C}^{\prime}}\left(V_{y_{2}}^{(1)}\right)\right]+m_{2} \frac{1}{2} \frac{\partial}{\partial t}\left[\frac{\partial V_{z_{2}}^{(2)}}{\partial \dot{w}_{1 C}^{\prime}}\left(V_{z_{2}}^{(1)}\right)\right]+\right. \\
& +I_{\eta_{2}} \frac{1}{2} \frac{\partial}{\partial t}\left[\frac{\partial \omega_{\eta_{2}}^{(2)}}{\partial \dot{w}_{1 C}^{\prime}}\left(\omega_{\eta_{2}}^{(1)}\right)\right]-m_{2} \frac{1}{2}\left[\frac{\partial V_{y_{2}}^{(2)}}{\partial w_{1 C}^{\prime}}\left(V_{y_{2}}^{(1)}\right)\right]-m_{2} \frac{1}{2}\left[\frac{\partial V_{z_{2}}^{(2)}}{\partial w_{1 C}^{\prime}}\left(V_{z_{2}}^{(1)}\right)\right]-
\end{aligned}
$$




$$
\begin{gathered}
-I_{\zeta_{2}} \frac{1}{2}\left[\frac{\partial \omega_{\zeta_{2}}^{(2)}}{\partial w_{1 C}^{\prime}}\left(\omega_{\zeta_{2}}^{(1)}\right)\right]-I_{\eta_{2}} \frac{1}{2} \frac{\partial \omega_{\eta_{2}}^{(2)}}{\partial w_{1 C}^{\prime}}\left(\omega_{\eta_{2}}^{(1)}\right)+D_{\eta_{2}} \frac{1}{2} \frac{\partial \rho_{\eta_{2}}^{(2)}}{\partial w_{1 C}^{\prime}}\left(\rho_{\eta_{2}}^{(1)}\right)+D_{\zeta_{2}} \frac{1}{2} \frac{\partial \rho_{\zeta_{2}}^{(2)}}{\partial w_{1 C}^{\prime}}\left(\rho_{\zeta_{2}}^{(1)}\right)- \\
\left.-M_{x, 2}\right\} d s_{2} \delta w_{1 C}^{\prime}=-\int_{0}^{l_{2}}\left\{m_{2}\left(\ddot{V}_{2} W_{2}+\dot{V}_{2} \dot{W}_{2}+s_{2} \dot{v}_{1 c}^{\prime} \dot{V}_{2}+s_{2} v_{1 c}^{\prime} \ddot{V}_{2}\right)+\right. \\
+m_{2}\left(-\ddot{W}_{2} V_{2}-\dot{W}_{2} \dot{V}_{2}+\ddot{W}_{2} w_{1 c}+\dot{W}_{2} \dot{W}_{1 c}\right)-I_{\eta_{2}} \delta_{3}\left(\ddot{W}_{2}^{\prime} \varphi_{1 c} \dot{W}_{2}^{\prime} \dot{\varphi}_{1 c}\right)-m_{2} s_{2} \dot{v}_{1 c}^{\prime} \dot{V}_{2}- \\
-m_{2} \dot{W}_{2} \dot{W}_{1 c}-I_{\zeta_{2}} \delta_{2}\left(\dot{V}_{2}^{\prime} \dot{W}_{2}^{\prime}+2 \dot{v}_{1 c}^{\prime} \dot{V}_{2}^{\prime}\right)-I_{\eta_{2}} \delta_{3}\left(-\dot{V}_{2}^{\prime} \dot{W}_{2}^{\prime}+\dot{\varphi}_{1 c} \dot{W}_{2}^{\prime}\right)- \\
\left.-D_{\eta_{2}} W_{2}^{\prime \prime} V_{2}^{\prime \prime}+D_{\zeta_{2}} W_{2}^{\prime \prime} V_{2}^{\prime \prime}-M_{x, 2}\right\} d s_{2} \delta w_{1 C}^{\prime} .
\end{gathered}
$$

Working with equation (51) and equation (22) leads to,

$$
\begin{gathered}
-\left.\frac{1}{2} \frac{\partial h_{U_{2}}}{\partial \phi_{2}^{\prime}}\right|_{S_{2}=0} \delta w_{1 C}^{\prime}=-\left.\left[\frac{1}{2} D_{\xi_{2}} \frac{\partial \rho_{\xi_{2}}^{(1)}}{\partial \phi_{2}^{\prime}}\left(\rho_{\xi_{2}}^{(1)}+\rho_{\xi_{2}}^{(2)}\right)\right]\right|_{S_{2}=0} \delta w_{1 C}^{\prime}= \\
=-\left.\left[D_{\xi_{2}}\left(\phi_{2}^{\prime}+V_{2}^{\prime \prime} W_{2}^{\prime}+v_{1 C}^{\prime} V_{2}^{\prime \prime}\right)\right]\right|_{S_{2}=0} \delta w_{1 C}^{\prime} .
\end{gathered}
$$

d) Torsional motion $\left(\varphi_{1}\right)$,

$$
-\left.\frac{1}{2} \frac{\partial h_{U_{1}}}{\partial \varphi_{1}^{\prime}}\right|_{S_{1}=l_{1}} \delta \varphi_{1 C}=-\left.\left[\frac{1}{2} D_{\xi_{1}} \frac{\partial \rho_{\xi_{1}}^{(1)}}{\partial \varphi_{1}^{\prime}}\left(\rho_{\xi_{1}}^{(1)}+\rho_{\xi_{1}}^{(2)}\right)\right]\right|_{s_{1}=l_{1}} \delta \varphi_{1 C}=-\left.\left[D_{\xi_{1}}\left(\varphi_{1}^{\prime}+v_{1}^{\prime \prime} w_{1}^{\prime}\right)\right]\right|_{s_{1}=l_{1}} \delta \varphi_{1 C},
$$

$$
\begin{gathered}
-\int_{0}^{l_{2}}\left(\frac{1}{2} \frac{\partial^{2} h_{T_{2}}}{\partial \dot{\varphi}_{1 C} \partial t}-\frac{1}{2} \frac{\partial h_{T_{2}}}{\partial \varphi_{1 C}}-\frac{1}{2} \frac{\partial h_{F_{2}}}{\partial \varphi_{1 C}}+\frac{1}{2} \frac{\partial h_{U_{2}}}{\partial \varphi_{1 C}}+s_{2} q_{y, 2}+M_{y, 2}\right) d s_{2} \delta \varphi_{1 C}= \\
=-\int_{0}^{l_{2}}\left\{m_{2} \frac{1}{2} \frac{\partial}{\partial t}\left[\frac{\partial V_{x_{2}}^{(2)}}{\partial \dot{\varphi}_{1 C}}\left(V_{x_{2}}^{(1)}\right)\right]+I_{\xi_{2}} \frac{1}{2} \frac{\partial}{\partial t}\left[\frac{\partial \omega_{\xi_{2}}^{(2)}}{\partial \dot{\varphi}_{1 C}}\left(\omega_{\xi_{2}}^{(1)}\right)\right]+I_{\eta_{2}} \frac{1}{2} \frac{\partial}{\partial t}\left[\frac{\partial \omega_{\eta_{2}}^{(2)}}{\partial \dot{\varphi}_{1 C}}\left(\omega_{\eta_{2}}^{(1)}\right)\right]-\right. \\
-m_{2} \frac{1}{2}\left[\frac{\partial V_{x_{2}}^{(2)}}{\partial \varphi_{1 C}}\left(V_{x_{2}}^{(1)}\right)\right]-m_{2} \frac{1}{2}\left[\frac{\partial V_{y_{2}}^{(2)}}{\partial \varphi_{1 C}}\left(V_{y_{2}}^{(1)}\right)\right]-I_{\xi_{2}} \frac{1}{2}\left[\frac{\partial \omega_{\xi_{2}}^{(2)}}{\partial \varphi_{1 C}}\left(\omega_{\xi_{2}}^{(1)}\right)\right]-I_{\eta_{2}} \frac{1}{2}\left[\frac{\partial \omega_{\eta_{2}}^{(2)}}{\partial \varphi_{1 C}}\left(\omega_{\eta_{2}}^{(1)}\right)\right]- \\
\left.-\lambda_{2}\left(V_{2}^{\prime}-\varphi_{1 C}\right)+s_{2} q_{y, 2}+M_{y, 2}\right\} d s_{2} \delta \varphi_{1 C}=-\int_{0}^{l_{2}}\left\{m _ { 2 } \left(-\dot{V}_{2} \dot{v}_{1 c}-V_{2} \ddot{v}_{1 c}+s_{2} \dot{\varphi}_{1 c} \dot{v}_{1 c}+s_{2} \varphi_{1 c} \ddot{v}_{1 c}+\right.\right. \\
\left.+\dot{w}_{1 c} \dot{v}_{1 c}+w_{1 c} \ddot{v}_{1 c}\right)-I_{\xi_{2}}\left(\ddot{\phi}_{2} W_{2}^{\prime}+\dot{\phi}_{2} \dot{W}_{2}^{\prime}+\ddot{\phi}_{2} v_{1 C}^{\prime}+\dot{\phi}_{2} \dot{v}_{1 C}^{\prime}\right)+ \\
+I_{\eta_{2}} \delta_{3}\left(\ddot{W}_{2}^{\prime} \phi_{2}+\dot{W}_{2}^{\prime} \dot{\phi}_{2}+\ddot{W}_{2}^{\prime} w_{1 c}^{\prime}+\dot{W}_{2}^{\prime} \dot{w}_{1 c}^{\prime}\right)-m_{2}\left(s_{2} \dot{\varphi}_{1 c} \dot{v}_{1 c}+\dot{v}_{1 c} \dot{w}_{1 c}\right)+m_{2} \dot{v}_{1 c} \dot{V}_{2}- \\
\left.-I_{\xi_{2}} \delta_{1} \dot{\phi}_{2} \dot{v}_{1 c}^{\prime}+I_{\eta_{2}} \delta_{3} \dot{W}_{2}^{\prime} \dot{w}_{1 c}^{\prime}-\lambda_{2}\left(V_{2}^{\prime}-\varphi_{1 C}\right)+s_{2} q_{y, 2}+M_{y, 2}\right\} d s_{2} \delta \varphi_{1 C} .
\end{gathered}
$$

Taking equation (50b) and equation (22) lead to,

$$
\left.\frac{1}{2} \frac{\partial h_{U_{2}}}{\partial V_{2}^{\prime \prime}}\right|_{S_{2}=0} \delta \varphi_{1 C}=\frac{1}{2}\left[D_{\xi_{2}} \frac{\partial \rho_{\xi_{2}}^{(2)}}{\partial V_{2}^{\prime \prime}}\left(\rho_{\xi_{2}}^{(1)}\right)+D_{\eta_{2}} \frac{\partial \rho_{\eta_{2}}^{(2)}}{\partial V_{2}^{\prime \prime}}\left(\rho_{\eta_{2}}^{(1)}\right)+\right.
$$




$$
\begin{gathered}
\left.+D_{\zeta_{2}} \frac{\partial \rho_{\zeta_{2}}^{(1)}}{\partial V_{2}^{\prime \prime}}\left(\rho_{\zeta_{2}}^{(1)}+\rho_{\zeta_{2}}^{(2)}\right)\right]\left.\right|_{S_{2}=0} \delta \varphi_{1 C}=\left\{\left.\left[D_{\xi_{2}}\left(W_{2}^{\prime} \phi_{2}^{\prime}+v_{1 C}^{\prime} \phi_{2}^{\prime}\right)\right]\right|_{S_{2}=0}-\right. \\
\left.-\left.\left[D_{\eta_{2}}\left(\phi_{2} W_{2}^{\prime \prime}+w_{1 C}^{\prime} W_{2}^{\prime \prime}\right)\right]\right|_{S_{2}=0}+\left.\left[D_{\zeta_{2}}\left(V_{2}^{\prime \prime}+\phi_{2} W_{2}^{\prime \prime}+w_{1 C}^{\prime} W_{2}^{\prime \prime}\right)\right]\right|_{S_{2}=0}\right\} \delta \varphi_{1 C} .
\end{gathered}
$$

-For the secondary beam,

e) axial motion $\left(u_{2}\right)$,

$$
\begin{gathered}
\frac{1}{2} \frac{\partial^{2} h_{T_{2}}}{\partial \dot{u}_{2}^{\prime} \partial t}-\frac{1}{2} \frac{\partial h_{T_{2}}}{\partial u_{2}^{\prime}}-\frac{1}{2} \frac{\partial^{2} h_{U_{2}}}{\partial u_{2}^{\prime \prime} \partial s_{2}}+\frac{1}{2} \frac{\partial h_{U_{2}}}{\partial u_{2}^{\prime}}+\left.\frac{1}{2} \frac{\partial h_{F_{2}}}{\partial u_{2}^{\prime}}\right|_{S_{2}=l_{2}}= \\
=\left.\left[I_{\eta_{2}} \delta_{3}\left(-W_{2}^{\prime} \ddot{W}_{2}^{\prime}+v_{1 c} \ddot{W}_{2}^{\prime}\right)\right]\right|_{S_{2}=l_{2}}+\left.\left[I_{\zeta_{2}} \delta_{2}\left(-\ddot{V}_{2}^{\prime} V_{2}^{\prime}+\varphi_{1 c} \ddot{V}_{2}^{\prime}\right)\right]\right|_{S_{2}=l_{2}}+ \\
+\left.\left\{\frac{\partial}{\partial s_{2}}\left[D_{\eta_{2}}\left(W_{2}^{\prime} W_{2}^{\prime \prime}+v_{1 c}^{\prime} W_{2}^{\prime \prime}\right)\right]\right\}\right|_{S_{2}=l_{2}}-\left.\left\{\frac{\partial}{\partial s_{2}}\left[D_{\zeta_{2}}\left(-V_{2}^{\prime} V_{2}^{\prime \prime}+\varphi_{1 c} V_{2}^{\prime \prime}\right)\right]\right\}\right|_{S_{2}=l_{2}}- \\
-\left.\left[D_{\eta_{2}} W_{2}^{\prime \prime 2}\right]\right|_{S_{2}=l_{2}}-\left.\left[D_{\zeta_{2}} V_{2}^{\prime \prime 2}\right]\right|_{S_{2}=l_{2}}-\left.\left[\lambda_{2}\left(1+u_{2}^{\prime}\right)\right]\right|_{S_{2}=l_{2}} .
\end{gathered}
$$

f) In-plane bending $\left(W_{2}\right)$,

$$
\begin{gathered}
\left.\left(-\frac{1}{2} \frac{\partial^{2} h_{T_{2}}}{\partial \dot{W}_{2}^{\prime} \partial t}+\frac{1}{2} \frac{\partial h_{T_{2}}}{\partial W_{2}^{\prime}}+\frac{1}{2} \frac{\partial h_{F_{2}}}{\partial W_{2}^{\prime}}+\frac{1}{2} \frac{\partial^{2} h_{U_{2}}}{\partial W_{2}^{\prime \prime} \partial s_{2}}-\frac{1}{2} \frac{\partial h_{U_{2}}}{\partial W_{2}^{\prime}}-M_{z, 2}\right)\right|_{S_{2}=l_{2}}= \\
=\left\{-\frac{1}{2} \frac{\partial}{\partial t}\left[I_{\zeta_{2}} \frac{\partial \omega_{\zeta_{2}}^{(2)}}{\partial \dot{W}_{2}^{\prime}}\left(\omega_{\zeta_{2}}^{(1)}\right)+I_{\eta_{2}} \frac{\partial \omega_{\eta_{2}}^{(1)}}{\partial \dot{W}_{2}^{\prime}}\left(\omega_{\eta_{2}}^{(1)}+\omega_{\eta_{2}}^{(2)}\right)\right]+\frac{1}{2}\left[I_{\xi_{2}} \frac{\partial \omega_{\xi_{2}}^{(2)}}{\partial W_{2}^{\prime}}\left(\omega_{\xi_{2}}^{(1)}\right)\right]-\right. \\
-\left[\lambda_{2}\left(W_{2}^{\prime}+v_{1 C}^{\prime}\right)\right]+\frac{1}{2} \frac{\partial}{\partial s_{2}}\left[D_{\eta_{2}} \frac{\partial \rho_{\eta_{2}}^{(1)}}{\partial W_{2}^{\prime \prime}}\left(\rho_{\eta_{2}}^{(1)}+\rho_{\eta_{2}}^{(2)}\right)+\right. \\
\left.\left.+D_{\zeta_{2}} \frac{\partial \rho_{\zeta_{2}}^{(2)}}{\partial W_{2}^{\prime \prime}}\left(\rho_{\zeta_{2}}^{(1)}\right)\right]-\frac{1}{2}\left[D_{\xi_{2}} \frac{\partial \rho_{\xi_{2}}^{(2)}}{\partial W_{2}^{\prime}}\left(\rho_{\xi_{2}}^{(1)}\right)\right]-M_{z, 2}\right\}= \\
=-\left.\left[I_{\zeta_{2}} \delta_{2}\left(\dot{\phi}_{2} \dot{V}_{2}^{\prime}+\phi_{2} \ddot{V}_{2}^{\prime}+\dot{w}_{1 c}^{\prime} \dot{V}_{2}^{\prime}+w_{1 c}^{\prime} \ddot{V}_{2}^{\prime}\right)\right]\right|_{S_{2}=l_{2}}+\left[I _ { \eta _ { 2 } } \delta _ { 3 } \left(-\ddot{W}_{2}^{\prime}+\dot{\phi}_{2} \dot{V}_{2}^{\prime}+\phi_{2} \ddot{V}_{2}^{\prime}+\dot{w}_{1 c}^{\prime} \dot{V}_{2}^{\prime}+\right.\right. \\
\left.\left.+w_{1 c}^{\prime} \ddot{V}_{2}^{\prime}-\dot{\phi}_{2} \dot{\varphi}_{1 c}-\phi_{2} \ddot{\varphi}_{1 c}-\dot{w}_{1 c}^{\prime} \dot{\varphi}_{1 c}-w_{1 c}^{\prime} \ddot{\varphi}_{1 c}+\ddot{w}_{1 c}^{\prime} \varphi_{1 c}+\dot{w}_{1 c}^{\prime} \dot{\varphi}_{1 c}\right)\right]\left.\right|_{S_{2}=l_{2}}+ \\
+\left.\left[I_{\xi_{2}}\left(\dot{V}_{2}^{\prime} \dot{\phi}_{2}-\dot{\varphi}_{1 c} \dot{\phi}_{2}\right)\right]\right|_{S_{2}=l_{2}}- \\
-\left.\left[\lambda_{2}\left(W_{2}^{\prime}+v_{1 C}^{\prime}\right)\right]\right|_{s_{2}=l_{2}}-\left.\left\{\frac{\partial}{\partial s_{2}} D_{\eta_{2}}\left[\left(-W_{2}^{\prime \prime}+\phi_{2} V_{2}^{\prime \prime}+w_{1 C}^{\prime} V_{2}^{\prime \prime}\right)\right]\right\}\right|_{S_{2}=l_{2}}+ \\
+\left.\left\{\frac{\partial}{\partial s_{2}}\left[D_{\zeta_{2}}\left(V_{2}^{\prime \prime} \phi_{2}+V_{2}^{\prime \prime} w_{1 C}^{\prime}\right)\right]\right\}\right|_{S_{2}=l_{2}}-\left[D_{\xi_{2}} \phi_{2}^{\prime} V_{2}^{\prime \prime}\right]_{S_{2}=l_{2}}-M_{z, 2}\left(l_{2}, t\right), \\
-\left.\frac{1}{2} \frac{\partial h_{U_{2}}}{\partial W_{2}^{\prime \prime}}\right|_{S_{2}=l_{2}}=-\left.\frac{1}{2}\left[D_{\eta_{2}} \frac{\partial \rho_{\eta_{2}}^{(1)}}{\partial W_{2}^{\prime \prime}}\left(\rho_{\eta_{2}}^{(1)}+\rho_{\eta_{2}}^{(2)}\right)+D_{\zeta_{2}} \frac{\partial \rho_{\zeta_{2}}^{(2)}}{\partial W_{2}^{\prime \prime}}\left(\rho_{\zeta_{2}}^{(1)}\right)\right]\right|_{s_{2}=l_{2}}=
\end{gathered}
$$




$$
=\left.\left[D_{\eta_{2}}\left(-W_{2}^{\prime \prime}+\phi_{2} V_{2}^{\prime \prime}+w_{1 C}^{\prime} V_{2}^{\prime \prime}\right)\right]\right|_{S_{2}=l_{2}}-\left.\left[D_{\zeta_{2}}\left(V_{2}^{\prime \prime} \phi_{2}+V_{2}^{\prime \prime} w_{1 C}^{\prime}\right)\right]\right|_{S_{2}=l_{2}} .
$$

g) Out-of-plane bending $\left(V_{2}\right)$,

$$
\begin{aligned}
& \left.\left(-\frac{1}{2} \frac{\partial^{2} h_{T_{2}}}{\partial \dot{V}_{2}^{\prime} \partial t}+\frac{1}{2} \frac{\partial h_{T_{2}}}{\partial V_{2}^{\prime}}+\frac{1}{2} \frac{\partial h_{F_{2}}}{\partial V_{2}^{\prime}}+\frac{1}{2} \frac{\partial^{2} h_{U_{2}}}{\partial V_{2}^{\prime \prime} \partial s_{2}}-\frac{1}{2} \frac{\partial h_{U_{2}}}{\partial V_{2}^{\prime}}-\frac{1}{2} \frac{\partial h_{U_{2}}}{\partial V_{2}^{\prime}}+M_{y, 2}\right)\right|_{S_{2}=l_{2}}= \\
& =\left\{-\frac{1}{2} \frac{\partial}{\partial t}\left[I_{\xi_{2}} \frac{\partial \omega_{\xi_{2}}^{(2)}}{\partial \dot{V}_{2}^{\prime}}\left(\omega_{\xi_{2}}^{(1)}\right)+I_{\zeta_{2}} \frac{\partial \omega_{\zeta_{2}}^{(1)}}{\partial \dot{V}_{2}^{\prime}}\left(\omega_{\zeta_{2}}^{(1)}+\omega_{\zeta_{2}}^{(2)}\right)+I_{\eta_{2}} \frac{\partial \omega_{\eta_{2}}^{(2)}}{\partial \dot{V}_{2}^{\prime}}\left(\omega_{\eta_{2}}^{(1)}\right)\right]+2 \frac{\partial}{\partial S_{2}}\left[I_{\zeta_{2}} \frac{\partial \omega_{\zeta_{2}}^{(3)}}{\partial V_{2}^{\prime}} \omega_{\zeta_{2}}^{(1)}\right]-\right. \\
& -\left[\lambda_{2}\left(V_{2}^{\prime}-\varphi_{1 C}\right)\right]+\frac{1}{2} \frac{\partial}{\partial s_{2}}\left[D_{\xi_{2}} \frac{\partial \rho_{\xi_{2}}^{(2)}}{\partial V_{2}^{\prime \prime}}\left(\rho_{\xi_{2}}^{(1)}\right)+D_{\eta_{2}} \frac{\partial \rho_{\eta_{2}}^{(2)}}{\partial V_{2}^{\prime \prime}}\left(\rho_{\eta_{2}}^{(1)}\right)+D_{\zeta_{2}} \frac{\partial \rho_{\zeta_{2}}^{(1)}}{\partial V_{2}^{\prime \prime}}\left(\rho_{\zeta_{2}}^{(1)}+\rho_{\zeta_{2}}^{(2)}\right)\right]- \\
& \left.-2 \frac{\partial}{\partial s_{2}}\left[D_{\zeta_{2}} \frac{\partial \rho_{\zeta_{2}}^{(3)}}{\partial V_{2}^{\prime}} \rho_{\zeta_{2}}^{(1)}\right]+M_{y, 2}\right\}\left.\right|_{S_{2}=l_{2}}= \\
& =-\left.\left[I_{\xi_{2}}\left(\dot{W}_{2}^{\prime} \dot{\phi}_{2}+W_{2}^{\prime} \ddot{\phi}_{2}+\dot{v}_{1 c}^{\prime} \dot{\phi}_{2}+v_{1 c}^{\prime} \ddot{\phi}_{2}\right)\right]\right|_{S_{2}=l_{2}}-\left[I _ { \zeta _ { 2 } } \delta _ { 2 } \left(\ddot{V}_{2}^{\prime}+\ddot{W}_{2}^{\prime} \phi_{2}+\dot{\phi}_{2} \dot{W}_{2}^{\prime}+\ddot{v}_{1 c}^{\prime} \phi_{2}+\dot{v}_{1 c}^{\prime} \dot{\phi}_{2}+\right.\right. \\
& \left.\left.+\ddot{W}_{2}^{\prime} w_{1 c}^{\prime}+\dot{W}_{2}^{\prime} \dot{W}_{1 c}^{\prime}+2 \ddot{v}_{1 c}^{\prime} w_{1 c}^{\prime}+2 \dot{v}_{1 c}^{\prime} \dot{W}_{1 c}^{\prime}\right)\right]\left.\right|_{s_{2}=l_{2}}+\left.\left[I_{\eta_{2}} \delta_{3}\left(\dot{\phi}_{2} \dot{W}_{2}^{\prime}+\phi_{2} \ddot{W}_{2}^{\prime}+\dot{w}_{1 c}^{\prime} \dot{W}_{2}^{\prime}+w_{1 c}^{\prime} \ddot{W}_{2}^{\prime}\right)\right]\right|_{s_{2}=l_{2}}- \\
& -\left.\left[\lambda_{2}\left(V_{2}^{\prime}-\varphi_{1 C}\right)\right]\right|_{s_{2}=l_{2}}+\left.\left\{\frac{\partial}{\partial s_{2}}\left[D_{\xi_{2}}\left(W_{2}^{\prime} \phi_{2}^{\prime}+v_{1 C}^{\prime} \phi_{2}^{\prime}\right)\right]\right\}\right|_{s_{2}=l_{2}}-\left.\left\{\frac{\partial}{\partial s_{2}}\left[D_{\eta_{2}}\left(\phi_{2} W_{2}^{\prime \prime}+w_{1 C}^{\prime} W_{2}^{\prime \prime}\right)\right]\right\}\right|_{s_{2}=l_{2}}+ \\
& +\left.\left\{\frac{\partial}{\partial s_{2}}\left[D_{\zeta_{2}}\left(V_{2}^{\prime \prime}+\phi_{2} W_{2}^{\prime \prime}+w_{1 C}^{\prime} W_{2}^{\prime \prime}\right)\right]\right\}\right|_{S_{2}=l_{2}}+M_{y, 2}\left(l_{2}, t\right)+\text { H.O.T. } \\
& -\left.\frac{1}{2} \frac{\partial h_{U_{2}}}{\partial V_{2}^{\prime \prime}}\right|_{S_{2}=l_{2}}=-\left.\frac{1}{2}\left[D_{\xi_{2}} \frac{\partial \rho_{\xi_{2}}^{(2)}}{\partial V_{2}^{\prime \prime}}\left(\rho_{\xi_{2}}^{(1)}\right)+D_{\eta_{2}} \frac{\partial \rho_{\eta_{2}}^{(2)}}{\partial V_{2}^{\prime \prime}}\left(\rho_{\eta_{2}}^{(1)}\right)+D_{\zeta_{2}} \frac{\partial \rho_{\zeta_{2}}^{(1)}}{\partial V_{2}^{\prime \prime}}\left(\rho_{\zeta_{2}}^{(1)}+\rho_{\zeta_{2}}^{(2)}\right)\right]\right|_{S_{2}=l_{2}}= \\
& =-\left.\left[D_{\xi_{2}}\left(W_{2}^{\prime} \phi_{2}^{\prime}+v_{1 C}^{\prime} \phi_{2}^{\prime}\right)\right]\right|_{s_{2}=l_{2}}+\left.\left[D_{\eta_{2}}\left(\phi_{2} W_{2}^{\prime \prime}+w_{1 C}^{\prime} W_{2}^{\prime \prime}\right)\right]\right|_{S_{2}=l_{2}}- \\
& -\left.\left[D_{\zeta_{2}}\left(V_{2}^{\prime \prime}+\phi_{2} W_{2}^{\prime \prime}+w_{1 C}^{\prime} W_{2}^{\prime \prime}\right)\right]\right|_{S_{2}=l_{2}} \text {. }
\end{aligned}
$$

h) Torsional motion $\left(\phi_{2}\right)$,

$$
-\left.\frac{1}{2} \frac{\partial h_{U_{2}}}{\partial \phi_{2}^{\prime}}\right|_{S_{2}=l_{2}}=-\left.\left[\frac{1}{2} D_{\xi_{2}} \frac{\partial \rho_{\xi_{2}}^{(1)}}{\partial \phi_{2}^{\prime}}\left(\rho_{\xi_{2}}^{(1)}+\rho_{\xi_{2}}^{(2)}\right)\right]\right|_{S_{2}=l_{2}}=-\left.\left[D_{\xi_{2}}\left(\phi_{2}^{\prime}+V_{2}^{\prime \prime} W_{2}^{\prime}+v_{1 C}^{\prime} V_{2}^{\prime \prime}\right)\right]\right|_{S_{2}=l_{2}} .
$$

\section{Appendix-C}

The Lagrange multipliers are determined in this section. Integration of the axial equation of motion for the primary beam of equation (24) leads to,

$$
\begin{gathered}
\left(1+u_{1}^{\prime}\right) \lambda_{1}+c_{\lambda_{1}}=\int_{0}^{s_{1}}\left(m_{1} \ddot{u}_{1}+c_{11} \dot{u}_{1}\right) d s_{1}+I_{\zeta_{1}} \delta_{3} v_{1}^{\prime} \ddot{v}_{1}^{\prime}+I_{\eta_{1}} \delta_{1} w_{1}^{\prime} \ddot{w}_{1}^{\prime}- \\
-\frac{\partial}{\partial s_{1}}\left[D_{\eta_{1}} w_{1}^{\prime} w_{1}^{\prime \prime}\right]-\frac{\partial}{\partial s_{1}}\left[D_{\zeta_{1}} v_{1}^{\prime} v_{1}^{\prime \prime}\right]+D_{\eta_{1}} w_{1}^{\prime \prime 2}+D_{\zeta_{1}} v_{1}^{\prime \prime 2}
\end{gathered}
$$


Then, by applying the boundary conditions for the axial equation of the primary beam, equation (52) one get,

$$
\begin{gathered}
c_{\lambda_{1}}=\int_{0}^{l_{1}}\left(m_{1} \ddot{u}_{1}+c_{11} \dot{u}_{1}\right) d s_{1}+\int_{0}^{l_{2}}\left[m_{2}\left(-\dot{v}_{1 c}^{\prime} \dot{v}_{1 c}-v_{1 c}^{\prime} \ddot{v}_{1 c}\right)-m_{2}\left(\dot{w}_{1 c}^{\prime} \dot{V}_{2}+w_{1 c}^{\prime} \ddot{V}_{2}\right)+\right. \\
\left.+m_{2} \dot{v}_{1 c}^{\prime} \dot{v}_{1 c}+m_{2} \dot{V}_{2} \dot{w}_{1 c}^{\prime}+q_{z, 2}\right] d s_{2}-\left\{\left.\left[I_{\zeta_{2}} \delta_{2}\left(\dot{\phi}_{2} \dot{V}_{2}^{\prime}+\phi_{2} \ddot{V}_{2}^{\prime}+\dot{w}_{1 c}^{\prime} \dot{V}_{2}^{\prime}+w_{1 c}^{\prime} \ddot{V}_{2}^{\prime}\right)\right]\right|_{S_{2}=0}+\right. \\
+\left[I _ { \eta _ { 2 } } \delta _ { 3 } \left(-\ddot{W}_{2}^{\prime}+\dot{\phi}_{2} \dot{V}_{2}^{\prime}+\phi_{2} \ddot{V}_{2}^{\prime}+\dot{w}_{1 c}^{\prime} \dot{V}_{2}^{\prime}+\right.\right. \\
\left.\left.+w_{1 c}^{\prime} \ddot{V}_{2}^{\prime}-\dot{\phi}_{2} \dot{\varphi}_{1 c}-\phi_{2} \ddot{\varphi}_{1 c}-\dot{w}_{1 c}^{\prime} \dot{\varphi}_{1 c}-w_{1 c}^{\prime} \ddot{\varphi}_{1 c}+\ddot{w}_{1 c}^{\prime} \varphi_{1 c}+\dot{w}_{1 c}^{\prime} \dot{\varphi}_{1 c}\right)\right]\left.\right|_{s_{2}=0}+ \\
+\left.\left[I_{\xi_{2}}\left(\dot{V}_{2}^{\prime} \dot{\phi}_{2}-\varphi_{1 c} \dot{\phi}_{2}\right)\right]\right|_{s_{2}=0}-\left.\left[\lambda_{2}\left(W_{2}^{\prime}+v_{1 c}^{\prime}\right)\right]\right|_{s_{2}=0}-\left.\left\{\frac{\partial}{\partial s_{2}}\left[D_{\eta_{2}}\left(-W_{2}^{\prime \prime}+\phi_{2} V_{2}^{\prime \prime} w_{1 c}^{\prime} V_{2}^{\prime \prime}\right)\right]\right\}\right|_{s_{2}=0}+ \\
\left.+\left.\left\{\frac{\partial}{\partial s_{2}}\left[D_{\zeta_{2}}\left(V_{2}^{\prime \prime} \phi_{2}+V_{2}^{\prime \prime} w_{1 C}^{\prime}\right)\right]\right\}\right|_{S_{2}=0}-\left.\left[D_{\xi_{2}} \phi_{2}^{\prime} V_{2}^{\prime \prime}\right]\right|_{s_{2}=0}-M_{z, 2}\left(l_{2}, t\right)\right\}= \\
=\int_{0}^{l_{1}}\left(m_{1} \ddot{u}_{1}+c_{11} \dot{u}_{1}\right) d s_{1}-\int_{0}^{l_{2}} q_{z, 2} d s_{2}+M_{z, 2}(0, t)+\mathrm{I}_{c_{\lambda_{1}}}^{(1)}+\mathrm{I}_{c_{\lambda_{1}}}^{(2)},
\end{gathered}
$$

with,

$$
\begin{gathered}
\mathrm{I}_{c_{\lambda_{1}}}^{(1)}=I_{\eta_{2}} \delta_{3} \ddot{W}_{2}^{\prime}(0, t)-D_{\eta_{2}} W_{2}^{\prime \prime \prime}(0, t)-\left.\left[\lambda_{2}^{(0)}\left(W_{2}^{\prime}+v_{1 C}^{\prime}\right)\right]\right|_{S_{2}=0}, \\
\mathrm{I}_{c_{\lambda_{1}}}^{(2)}=\int_{0}^{l_{2}}\left[m_{2}\left(-\dot{v}_{1 c}^{\prime} \dot{v}_{1 c}-v_{1 c}^{\prime} \ddot{v}_{1 c}\right)-m_{2}\left(\dot{w}_{1 c}^{\prime} \dot{V}_{2}+w_{1 c}^{\prime} \ddot{V}_{2}\right)+m_{2} \dot{v}_{1 c}^{\prime} \dot{v}_{1 c}+m_{2} \dot{V}_{2} \dot{w}_{1 c}^{\prime}\right] d s_{2}- \\
-\left\{\left.\left[I_{\zeta_{2}} \delta_{2}\left(\dot{\phi}_{2} \dot{V}_{2}^{\prime}+\phi_{2} \ddot{V}_{2}^{\prime}+\dot{w}_{1 c}^{\prime} \dot{V}_{2}^{\prime}+w_{1 c}^{\prime} \ddot{V}_{2}^{\prime}\right)\right]\right|_{S_{2}=0}+\left[I _ { \eta _ { 2 } } \delta _ { 3 } \left(\dot{\phi}_{2} \dot{V}_{2}^{\prime}+\phi_{2} \ddot{V}_{2}^{\prime}+\dot{w}_{1 c}^{\prime} \dot{V}_{2}^{\prime}+w_{1 c}^{\prime} \ddot{V}_{2}^{\prime}-\right.\right.\right. \\
\left.\left.-\dot{\phi}_{2} \dot{\varphi}_{1 c}-\phi_{2} \ddot{\varphi}_{1 c}-\dot{w}_{1 c}^{\prime} \dot{\varphi}_{1 c}-w_{1 c}^{\prime} \ddot{\varphi}_{1 c}+\ddot{w}_{1 c}^{\prime} \varphi_{1 c}+\dot{w}_{1 c}^{\prime} \dot{\varphi}_{1 c}\right)\right]\left.\right|_{S_{2}=0}+ \\
+\left.\left\{I_{\xi_{2}}\left(\dot{V}_{2}^{\prime} \dot{\phi}_{2}-\dot{\varphi}_{1 c} \dot{\phi}_{2}\right)\right\}\right|_{S_{2}=0}-\left.\left[\lambda_{2}^{(1)}\left(W_{2}^{\prime}+v_{1 C}^{\prime}\right)\right]\right|_{S_{2}=0}-\left.\left\{\frac{\partial}{\partial s_{2}}\left[D_{\eta_{2}}\left(\phi_{2} V_{2}^{\prime \prime}+w_{1 C}^{\prime} V_{2}^{\prime \prime}\right)\right]\right\}\right|_{S_{2}=0}+ \\
+\left.\left\{\frac{\partial}{\partial s_{2}}\left[D_{\zeta_{2}}\left(V_{2}^{\prime \prime} \phi_{2}+V_{2}^{\prime \prime} w_{1 C}^{\prime}\right)\right]\right\}\right|_{s_{2}=0}-\left.\left[D_{\xi_{2}} \phi_{2}^{\prime} V_{2}^{\prime \prime}\right]\right|_{S_{2}=0} .
\end{gathered}
$$

Therefore, by using (C.2), equation (C.1) takes the form,

$$
\begin{aligned}
\lambda_{1}=\frac{1}{\left(1+u_{1}^{\prime}\right)} & \left\{\int_{l_{1}}^{s_{1}}\left(m_{1} \ddot{u}_{1}+c_{11} \dot{u}_{1}\right) d s_{1}+I_{\zeta_{1}} \delta_{3} v_{1}^{\prime} \ddot{v}_{1}^{\prime}+I_{\eta_{1}} \delta_{1} w_{1}^{\prime} \ddot{w}_{1}^{\prime}-\frac{\partial}{\partial s_{1}}\left[D_{\eta_{1}} w_{1}^{\prime} w_{1}^{\prime \prime}\right]-\right. \\
& \left.-\frac{\partial}{\partial s_{1}}\left[D_{\zeta_{1}} v_{1}^{\prime} v_{1}^{\prime \prime}\right]-M_{z, 2}(0, t)-\int_{0}^{l_{2}} q_{z, 2} d s_{2}-\mathrm{I}_{c_{\lambda_{1}}}^{(1)}-\mathrm{I}_{c_{\lambda_{1}}}^{(2)}\right\}
\end{aligned}
$$

or, by considering also the expansion of the fraction in series form,

$$
\frac{1}{\left(1+u_{1}^{\prime}\right)}=1-u_{1}^{\prime}+\left(u_{1}^{\prime}\right)^{2}-\left(u_{1}^{\prime}\right)^{3},
$$

whereby $u_{1}^{\prime}$ is of second order, then, 


$$
\begin{gathered}
\lambda_{1}=\left\{\lambda_{1}^{(0)}\right\}+\left\{\lambda_{1}^{(1)}\right\}+\left\{\lambda_{1}^{(2)}\right\}=\left\{-M_{z, 2}(0, t)-\int_{0}^{l_{2}} q_{z, 2} d s_{2}\right\}+\left\{-\mathrm{I}_{c_{\lambda_{1}}}^{(1)}\right\}+ \\
+\left\{\int_{l_{1}}^{s_{1}} c_{11} \dot{u}_{1} d s_{1}+M_{z, 2}(0, t) u_{1}^{\prime}+\int_{0}^{l_{2}} q_{z, 2} d s_{2} u_{1}^{\prime}+\right. \\
\left.+\int_{l_{1}}^{s_{1}} m_{1} \ddot{u}_{1} d s_{1}-\mathrm{I}_{c_{\lambda_{1}}}^{(2)}+I_{\zeta_{1}} \delta_{3} v_{1}^{\prime} \ddot{v}_{1}^{\prime}+I_{\eta_{1}} \delta_{1} w_{1}^{\prime} \ddot{w}_{1}^{\prime}-\frac{\partial}{\partial s_{1}}\left[D_{\eta_{1}} w_{1}^{\prime} w_{1}^{\prime \prime}\right]-\frac{\partial}{\partial s_{1}}\left[D_{\zeta_{1}} v_{1}^{\prime} v_{1}^{\prime \prime}\right]\right\} .
\end{gathered}
$$

The Lagrange multiplier for the secondary beam can be determined similarly, with spatial integration of the equation (28), which leads to,

$$
\begin{gathered}
\lambda_{2}\left(1+u_{2}^{\prime}\right)+\frac{\partial}{\partial s_{2}}\left[D_{\eta_{2}}\left(W_{2}^{\prime} W_{2}^{\prime \prime} v_{1 c}^{\prime} W_{2}^{\prime \prime}\right)\right]-\frac{\partial}{\partial s_{2}}\left[D_{\zeta_{2}}\left(-V_{2}^{\prime} V_{2}^{\prime \prime}+\varphi_{1 c} V_{2}^{\prime \prime}\right)\right]-D_{\eta_{2}} W_{2}^{\prime \prime 2}- \\
-D_{\zeta_{2}} V_{2}^{\prime \prime 2}+I_{\eta_{2}} \delta_{3}\left(-W_{2}^{\prime} \ddot{W}_{2}^{\prime}+v_{1 c} \ddot{W}_{2}^{\prime}\right)+I_{\zeta_{2}} \delta_{2}\left(-\ddot{V}_{2}^{\prime} V_{2}^{\prime}+\varphi_{1 c} \ddot{V}_{2}^{\prime}\right)+c_{\lambda_{2}}= \\
=\int_{0}^{s_{2}}\left\{m _ { 2 } \left(\ddot{v}_{1 c}+\ddot{u}_{2}+\ddot{v}_{1 c}^{\prime} W_{2}+\dot{v}_{1 c}^{\prime} \dot{W}_{2}+s_{2} \ddot{v}_{1 c}^{\prime} v_{1 c}^{\prime}+s_{2} \dot{v}_{1 c}^{\prime 2}-\right.\right. \\
-\ddot{\varphi}_{1 c} V_{2}-\dot{\varphi}_{1 c} \dot{V}_{2}+s_{2} \dot{\varphi}_{1 c}^{2}+s_{2} \varphi_{1 c} \ddot{\varphi}_{1 c}+\dot{\varphi}_{1 c} \dot{w}_{1 c}+\varphi_{1 c} \ddot{w}_{1 c}+\ddot{\varphi}_{1 c} w_{1 c}+\dot{\varphi}_{1 c} \dot{w}_{1 c}+ \\
+\dot{W}_{2} \varphi_{1 c} \dot{w}_{1 c}^{\prime}+W_{2} \dot{\varphi}_{1 c} \dot{w}_{1 c}^{\prime}+W_{2} \varphi_{1 c} \ddot{w}_{1 c}^{\prime}+\ddot{w}_{1 c}^{\prime} s_{2} \varphi_{1 c} v_{1 c}^{\prime}+\dot{w}_{1 c}^{\prime} s_{2} \dot{\varphi}_{1 c} v_{1 c}^{\prime}+\dot{w}_{1 c}^{\prime} s_{2} \varphi_{1 c} \dot{v}_{1 c}^{\prime}- \\
-\dot{V}_{2} \dot{v}_{1 c}^{\prime} w_{1 c}^{\prime}-V_{2} \ddot{v}_{1 c}^{\prime} w_{1 c}^{\prime}-V_{2} \dot{v}_{1 c}^{\prime} \dot{w}_{1 c}^{\prime}+s_{2} \dot{\varphi}_{1 c} \dot{v}_{1 c}^{\prime} w_{1 c}^{\prime}+s_{2} \varphi_{1 c} \ddot{v}_{1 c}^{\prime} w_{1 c}^{\prime}+s_{2} \varphi_{1 c} \dot{v}_{1 c}^{\prime} \dot{w}_{1 c}^{\prime}+ \\
+\ddot{v}_{1 c}^{\prime} w_{1 c}^{\prime} w_{1 c}+\dot{v}_{1 c}^{\prime} \dot{w}_{1 c}^{\prime} w_{1 c}+\dot{v}_{1 c}^{\prime} w_{1 c}^{\prime} \dot{w}_{1 c}-\dot{v}_{1 c}^{\prime} \dot{u}_{1 c}-v_{1 c}^{\prime} \ddot{u}_{1 c}-\varphi_{1 c} \dot{\varphi}_{1 c} \dot{v}_{1 c}-\frac{\varphi_{1 c}^{2}}{2} \ddot{v}_{1 c}- \\
\left.-v_{1 c}^{\prime} \dot{v}_{1 c}^{\prime} \dot{v}_{1 c}-\frac{v_{1 c}^{\prime 2}}{2} \ddot{v}_{1 c}\right)-m_{2}\left(\dot{V}_{2} \dot{\varphi}_{1 c}-\varphi_{1 c} \dot{v}_{1 c} \dot{\varphi}_{1 c}+W_{2} \dot{w}_{1 c}^{\prime} \dot{\varphi}_{1 c}+s_{2} \dot{v}_{1 c}^{\prime} w_{1 c}^{\prime} \dot{\varphi}_{1 c}+s_{2} v_{1 c}^{\prime} \dot{w}_{1 c}^{\prime} \dot{\varphi}_{1 c}\right)- \\
\left.-m_{2}\left(-\dot{W}_{2} \dot{v}_{1 c}^{\prime}-v_{1 c}^{\prime} \dot{v}_{1 c} \dot{v}_{1 c}^{\prime}-w_{1 c}^{\prime} \dot{w}_{1 c} \dot{v}_{1 c}^{\prime}+V_{2} \dot{w}_{1 c}^{\prime} \dot{v}_{1 c}^{\prime}-w_{1 c} \dot{w}_{1 c}^{\prime} \dot{v}_{1 c}^{\prime}\right)+c_{21} \dot{u}_{2}\right\} d s_{2} .
\end{gathered}
$$

Using also the boundary condition of equation (58) then,

$$
\begin{gathered}
c_{\lambda_{2}}=\int_{0}^{l_{2}}\left\{m _ { 2 } \left(\ddot{v}_{1 c}+\ddot{u}_{2}+\ddot{v}_{1 c}^{\prime} W_{2}+\dot{v}_{1 c}^{\prime} \dot{W}_{2}+s_{2} \ddot{v}_{1 c}^{\prime} v_{1 c}^{\prime}+s_{2} \dot{v}_{1 c}^{\prime 2}-\right.\right. \\
-\ddot{\varphi}_{1 c} V_{2}-\dot{\varphi}_{1 c} \dot{V}_{2}+s_{2} \dot{\varphi}_{1 c}^{2}+s_{2} \varphi_{1 c} \ddot{\varphi}_{1 c}+\dot{\varphi}_{1 c} \dot{w}_{1 c}+\varphi_{1 c} \ddot{w}_{1 c}+\ddot{\varphi}_{1 c} w_{1 c}+\dot{\varphi}_{1 c} \dot{w}_{1 c}+ \\
+\dot{W}_{2} \varphi_{1 c} \dot{w}_{1 c}^{\prime}+W_{2} \dot{\varphi}_{1 c} \dot{w}_{1 c}^{\prime}+W_{2} \varphi_{1 c} \ddot{w}_{1 c}^{\prime}+\ddot{w}_{1 c}^{\prime} s_{2} \varphi_{1 c} v_{1 c}^{\prime}+\dot{w}_{1 c}^{\prime} s_{2} \dot{\varphi}_{1 c} v_{1 c}^{\prime}+\dot{w}_{1 c}^{\prime} s_{2} \varphi_{1 c} \dot{v}_{1 c}^{\prime}- \\
-\dot{V}_{2} \dot{v}_{1 c}^{\prime} w_{1 c}^{\prime}-V_{2} \ddot{v}_{1 c}^{\prime} w_{1 c}^{\prime}-V_{2} \dot{v}_{1 c}^{\prime} \dot{w}_{1 c}^{\prime}+s_{2} \dot{\varphi}_{1 c} \dot{v}_{1 c}^{\prime} w_{1 c}^{\prime}+s_{2} \varphi_{1 c} \ddot{v}_{1 c}^{\prime} w_{1 c}^{\prime}+s_{2} \varphi_{1 c} \dot{v}_{1 c}^{\prime} \dot{w}_{1 c}^{\prime}+ \\
+\ddot{v}_{1 c}^{\prime} w_{1 c}^{\prime} w_{1 c}+\dot{v}_{1 c}^{\prime} \dot{w}_{1 c}^{\prime} w_{1 c}+\dot{v}_{1 c}^{\prime} w_{1 c}^{\prime} \dot{w}_{1 c}-\dot{v}_{1 c}^{\prime} \dot{u}_{1 c}-v_{1 c}^{\prime} \ddot{u}_{1 c}-\varphi_{1 c} \dot{\varphi}_{1 c} \dot{v}_{1 c}-\frac{\varphi_{1 c}^{2}}{2} \ddot{v}_{1 c}- \\
\left.-v_{1 c}^{\prime} \dot{v}_{1 c}^{\prime} \dot{v}_{1 c}-\frac{v_{1 c}^{\prime}}{2} \ddot{v}_{1 c}\right)-m_{2}\left(\dot{V}_{2} \dot{\varphi}_{1 c}-\varphi_{1 c} \dot{v}_{1 c} \dot{\varphi}_{1 c}+W_{2} \dot{w}_{1 c}^{\prime} \dot{\varphi}_{1 c}+s_{2} \dot{v}_{1 c}^{\prime} w_{1 c}^{\prime} \dot{\varphi}_{1 c}+s_{2} v_{1 c}^{\prime} \dot{w}_{1 c}^{\prime} \dot{\varphi}_{1 c}\right)- \\
\left.-m_{2}\left(-\dot{W}_{2} \dot{v}_{1 c}^{\prime}-v_{1 c}^{\prime} \dot{v}_{1 c} \dot{v}_{1 c}^{\prime}-w_{1 c}^{\prime} \dot{w}_{1 c} \dot{v}_{1 c}^{\prime}+V_{2} \dot{w}_{1 c}^{\prime} \dot{v}_{1 c}^{\prime}-w_{1 c} \dot{w}_{1 c}^{\prime} \dot{v}_{1 c}^{\prime}\right)+c_{21} \dot{u}_{2}\right\} d s_{2} .
\end{gathered}
$$

Finally, using equation (C.6) then equation (C.5) becomes,

$$
\lambda_{2}=\frac{1}{\left(1+u_{2}^{\prime}\right)} \int_{l_{2}}^{s_{2}}\left\{m _ { 2 } \left(\ddot{v}_{1 c}+\ddot{u}_{2}+\ddot{v}_{1 c}^{\prime} W_{2}+\dot{v}_{1 c}^{\prime} \dot{W}_{2}+s_{2} \ddot{v}_{1 c}^{\prime} v_{1 c}^{\prime}+s_{2} \dot{v}_{1 C}^{\prime 2}-\right.\right.
$$




$$
\begin{gathered}
-\ddot{\varphi}_{1 c} V_{2}-\dot{\varphi}_{1 c} \dot{V}_{2}+s_{2} \dot{\varphi}_{1 c}^{2}+s_{2} \varphi_{1 c} \ddot{\varphi}_{1 c}+\dot{\varphi}_{1 c} \dot{w}_{1 c}+\varphi_{1 c} \ddot{w}_{1 c}+\ddot{\varphi}_{1 c} w_{1 c}+\dot{\varphi}_{1 c} \dot{w}_{1 c}+ \\
+\dot{W}_{2} \varphi_{1 c} \dot{w}_{1 c}^{\prime}+W_{2} \dot{\varphi}_{1 c} \dot{w}_{1 c}^{\prime}+W_{2} \varphi_{1 c} \ddot{w}_{1 c}^{\prime}+\ddot{w}_{1 c}^{\prime} s_{2} \varphi_{1 c} v_{1 c}^{\prime}+\dot{w}_{1 c}^{\prime} s_{2} \dot{\varphi}_{1 c} v_{1 c}^{\prime}+\dot{w}_{1 c}^{\prime} s_{2} \varphi_{1 c} \dot{v}_{1 c}^{\prime}- \\
-\dot{V}_{2} \dot{v}_{1 c}^{\prime} w_{1 c}^{\prime}-V_{2} \ddot{v}_{1 c}^{\prime} w_{1 c}^{\prime}-V_{2} \dot{v}_{1 c}^{\prime} \dot{w}_{1 c}^{\prime}+s_{2} \dot{\varphi}_{1 c} \dot{v}_{1 c}^{\prime} w_{1 c}^{\prime}+s_{2} \varphi_{1 c} \ddot{v}_{1 c}^{\prime} w_{1 c}^{\prime}+s_{2} \varphi_{1 c} \dot{v}_{1 c}^{\prime} \dot{w}_{1 c}^{\prime}+ \\
+\ddot{v}_{1 c}^{\prime} w_{1 c}^{\prime} w_{1 c}+\dot{v}_{1 c}^{\prime} \dot{w}_{1 c}^{\prime} w_{1 c}+\dot{v}_{1 c}^{\prime} w_{1 c}^{\prime} \dot{w}_{1 c}-\dot{v}_{1 c}^{\prime} \dot{u}_{1 c}-v_{1 c}^{\prime} \ddot{u}_{1 c}-\varphi_{1 c} \dot{\varphi}_{1 c} \dot{v}_{1 c}-\frac{\varphi_{1 c}^{2}}{2} \ddot{v}_{1 c}- \\
\left.-v_{1 c}^{\prime} \dot{v}_{1 c}^{\prime} \dot{v}_{1 c}-\frac{v_{1 c}^{\prime 2}}{2} \ddot{v}_{1 c}\right)-m_{2}\left(\dot{V}_{2} \dot{\varphi}_{1 c}-\varphi_{1 c} \dot{v}_{1 c} \dot{\varphi}_{1 c}+W_{2} \dot{w}_{1 c}^{\prime} \dot{\varphi}_{1 c}+s_{2} \dot{v}_{1 c}^{\prime} w_{1 c}^{\prime} \dot{\varphi}_{1 c}+s_{2} v_{1 c}^{\prime} \dot{w}_{1 c}^{\prime} \dot{\varphi}_{1 c}\right)- \\
\left.-m_{2}\left(-\dot{W}_{2} \dot{v}_{1 c}^{\prime}-v_{1 c}^{\prime} \dot{v}_{1 c} \dot{v}_{1 c}^{\prime}-w_{1 c}^{\prime} \dot{w}_{1 c} \dot{v}_{1 c}^{\prime}+V_{2} \dot{w}_{1 c}^{\prime} \dot{v}_{1 c}^{\prime}-w_{1 c} \dot{w}_{1 c}^{\prime} \dot{v}_{1 c}^{\prime}\right)+c_{21} \dot{u}_{2}\right\} d s_{2}= \\
\frac{1}{\left(1+u_{2}^{\prime}\right)}\left(\mathrm{I}_{\lambda_{2}}^{(0)}+\mathrm{I}_{\lambda_{2}}^{(1)}+\mathrm{I}_{\lambda_{2}}^{(2)}-\frac{\partial}{\partial s_{2}}\left[D_{\eta_{2}}\left(W_{2}^{\prime} W_{2}^{\prime \prime}+v_{1 c}^{\prime} W_{2}^{\prime \prime}\right)\right]+\frac{\partial}{\partial s_{2}}\left[D_{\zeta_{2}}\left(-V_{2}^{\prime} V_{2}^{\prime \prime}+\varphi_{1 c} V_{2}^{\prime \prime}\right)\right]+\right. \\
\left.+D_{\eta_{2}} W_{2}^{\prime \prime 2}+D_{\zeta_{2}} V_{2}^{\prime \prime 2}-I_{\eta_{2}} \delta_{3}\left(-W_{2}^{\prime} \ddot{W}_{2}^{\prime}+v_{1 c} \ddot{W}_{2}^{\prime}\right)-I_{\zeta_{2}} \delta_{2}\left(-\ddot{V}_{2}^{\prime} V_{2}^{\prime}+\varphi_{1 c} \ddot{V}_{2}^{\prime}\right)\right),
\end{gathered}
$$

with,

$$
\begin{gathered}
\mathrm{I}_{\lambda_{2}}^{(0)}=0, \\
\mathrm{I}_{\lambda_{2}}^{(1)}=m_{2}\left(s_{2}-l_{2}\right) \ddot{v}_{1 c}, \\
\mathrm{I}_{\lambda_{2}}^{(2)}=\int_{l_{2}}^{s_{2}} c_{21} \dot{u}_{2} d s_{2}+\int_{l_{2}}^{s_{2}}\left\{m _ { 2 } \left(\ddot{u}_{2}+\ddot{v}_{1 c}^{\prime} W_{2}+\dot{v}_{1 c}^{\prime} \dot{W}_{2}+s_{2} \ddot{v}_{1 c}^{\prime} v_{1 c}^{\prime}+s_{2} \dot{v}_{1 c}^{\prime 2}-\right.\right. \\
\left.-\ddot{\varphi}_{1 c} V_{2}-\dot{\varphi}_{1 c} \dot{V}_{2}+s_{2} \dot{\varphi}_{1 c}^{2}+s_{2} \varphi_{1 c} \ddot{\varphi}_{1 c}+\dot{\varphi}_{1 c} \dot{w}_{1 c}+\varphi_{1 c} \ddot{W}_{1 c}+\ddot{\varphi}_{1 c} w_{1 c}+\dot{\varphi}_{1 c} \dot{W}_{1 c}\right)- \\
\left.-m_{2} \dot{V}_{2} \dot{\varphi}_{1 c}+m_{2} \dot{W}_{2} \dot{v}_{1 c}^{\prime}\right\} d s_{2} .
\end{gathered}
$$

An expansion of the fraction and a separation by the different orders of nonlinearity using equations (C.8a-c) leads to equation (C.7) of the form,

$$
\begin{gathered}
\lambda_{2}=\left\{\lambda_{2}^{(0)}\right\}+\left\{\lambda_{2}^{(1)}\right\}+\left\{\lambda_{2}^{(2)}\right\}=\left\{\mathrm{I}_{\lambda_{2}}^{(0)}\right\}+\left\{\mathrm{I}_{\lambda_{2}}^{(1)}\right\}+\left\{-\mathrm{I}_{\lambda_{2}}^{(0)} u_{2}^{\prime}+\mathrm{I}_{\lambda_{2}}^{(2)}-\right. \\
-\frac{\partial}{\partial s_{2}}\left[D_{\eta_{2}}\left(W_{2}^{\prime} W_{2}^{\prime \prime}+v_{1 c}^{\prime} W_{2}^{\prime \prime}\right)\right]+\frac{\partial}{\partial s_{2}}\left[D_{\zeta_{2}}\left(-V_{2}^{\prime} V_{2}^{\prime \prime}+\varphi_{1 c} V_{2}^{\prime \prime}\right)\right]+ \\
\left.+D_{\eta_{2}} W_{2}^{\prime \prime^{2}}+D_{\zeta_{2}} V_{2}^{\prime \prime}-I_{\eta_{2}} \delta_{3}\left(-W_{2}^{\prime} \ddot{W}_{2}^{\prime}+v_{1 c} \ddot{W}_{2}^{\prime}\right)-I_{\zeta_{2}} \delta_{2}\left(-\ddot{V}_{2}^{\prime} V_{2}^{\prime}+\varphi_{1 c} \ddot{V}_{2}^{\prime}\right)\right\}
\end{gathered}
$$

